\title{
Spatial Reorientation Experiments for NMR of Solids and Partially Oriented Liquids
}

\author{
Rachel W. Martin ${ }^{\mathrm{a}, \mathrm{b}, *}$, John E. Kelly ${ }^{\mathrm{a}}$, Kelsey A. Collier ${ }^{\mathrm{c}}$ \\ ${ }^{a}$ Department of Chemistry, University of California, Irvine 92697-2025 \\ ${ }^{b}$ Department of Molecular Biology and Biochemistry, University of California, Irvine \\ 92697-3900 \\ ${ }^{c}$ Department of Physics and Astronomy, University of California, Irvine 92697-4575
}

\begin{abstract}
Motional reorientation experiments are extensions of Magic Angle Spinning (MAS) where the rotor axis is changed in order to average out, reintroduce, or scale anisotropic interactions, (e.g. dipolar couplings, quadrupolar interactions or chemical shift anisotropies). This review focuses on Variable Angle Spinning (VAS), Switched Angle Spinning (SAS), and Dynamic Angle Spinning (DAS), all of which involve spinning at two or more different angles sequentially, either in successive experiments or during a multidimensional experiment. In all of these experiments, anisotropic terms in the Hamiltonian are scaled by changing the orientation of the spinning sample relative to the static magnetic field. These experiments vary in experimental complexity and instrumentation requirements. In VAS, many one-dimensional spectra are collected as a function of spinning angle. In SAS, dipolar couplings and/or chemical shift anisotropies are reintroduced by switching the sample between two different angles, often $0^{\circ}$ or $90^{\circ}$ and the magic angle, yielding a two-dimensional isotropic-anisotropic correlation spectrum. Dynamic Angle Spinning (DAS) is a related experiment that is used to simultaneously average out the first- and second-order quadrupolar interactions, which cannot be accomplished by spinning at any unique rotor angle in physical space. Although motional reorientation experiments generally require specialized instrumentation and data analysis schemes, some are accessible with only minor modification of standard MAS probes. In this review, the mechanics of each type of experiment is described, with representative examples. Cur-
\end{abstract}

*rwmartin@uci.edu

Preprint submitted to Progress in Nuclear Magnetic Resonance SpectroscopyOctober 12, 2015 
rent and historical probe and coil designs are discussed from the standpoint of how each one accomplishes the particular objectives of the experiment(s) it was designed to perform. Finally, applications to inorganic materials and liquid crystals, which present very different experimental challenges, are discussed. The review concludes with perspectives on how motional reorientation experiments can be applied to current problems in chemistry, molecular biology, and materials science, given the many advances in high-field NMR magnets, fast spinning, and sample preparation realized in recent years.

Keywords: Variable Angle Spinning, Switched Angle Spinning, Dynamic Angle Spinning, instrumentation, materials, liquid crystals

\section{Introduction}

Solid-state NMR is the structural method of choice for investigating noncrystalline solids, semi-solids, and liquid crystals. Here, the anisotropic interactions that are averaged out in isotropic liquids by molecular tumbling, including magnetic susceptibility differences, chemical shift anisotropy, quadrupolar interactions, and dipolar couplings, are present to a greater or lesser degree depending on the order parameter. Spatially-dependent anisotropic interactions are both the greatest strength and the biggest challenge of solid-state NMR; they provide exquisitely detailed local information about the chemical environment of the nuclear spins at the expense of significantly complicating the spectra. Anisotropic interactions are usually scaled or removed by a combination of Magic Angle Spinning (MAS) and radio frequency (rf) decoupling. Because completely removing these interactions results in the loss of valuable orientational information that is not available from liquids experiments, the heteronuclear dipolar interactions or chemical shift anisotropies are frequently selectively reintroduced using specialized pulse sequences.

Motional reorientation experiments represent an alternative strategy where the anisotropic interaction terms in the Hamiltonian are modified by changing the orientation of the sample in physical space, in contrast to the more common methods involving modulation of these interactions in spin-space via rotor-synchronized pulse sequences. In VAS, many one-dimensional spectra are collected as a function of spinning angle. In SAS, the structural information from the dipolar couplings and/or chemical shift anisotropies 
is recovered by switching the sample between two different angles, yielding a two-dimensional NMR spectrum. Thus, SAS measures the isotropic spectrum that is needed to assign the resonances of different chemical sites, while recovering valuable anisotropic information that would be lost in a simple MAS experiment. Dynamic Angle Spinning (DAS) is a special case of SAS for quadrupolar nuclei in which the two angles are chosen to average out the first- and second-order quadrupolar interactions. In the literature, DAS, SAS, and VAS are often used interchangeably, along with other terms such as angle-flipping. For purposes of this review, VAS describes a series of 1D experiments collected at different spinning angles, while SAS is a two-dimensional experiment where the indirect and direct dimensions are collected at different angles, often but not necessarily off the magic angle during the evolution period and on the magic angle during the detection period of the pulse sequence. DAS is reserved for the particular case where the angles are chosen to eliminate the first-order and second-order parts of the quadrupolar interaction. The problem posed by the second-order interaction is that its spatial dependence is described by a fourth-rank tensor, such that spinning about any one angle in physical space is insufficient to perform complete spatial averaging, as shown in Figure 1. The spins evolve at a rotor angle $\beta_{1}$ during time $t_{1}$, the magnetization is stored along the $z$-axis, and then the signal is collected at angle $\beta_{2}$ during time $t_{2}$. Given the appropriate choice of spinning angles (usually $30.6^{\circ}$ and $70.1^{\circ}$ ) and evolution times, evolution under the second- and fourth-rank terms during the second evolution period will cancel that from the first, producing an isotropic spectrum in the indirect dimension, correlated with the anisotropic lineshape in the direct dimension; refinements include pure absorption mode lineshapes [1] and suppression of sidebands [2]. DAS is experimentally quite different from Double Rotaton (DOR), in which the sample, contained within an inner and outer rotor, is spun about two axes, simultaneously averaging out both second-rank and fourth-rank spatial tensors, although in practice similar information is obtained.

These methods are the subject of several excellent review articles and perspective pieces that focus on different aspects of the methodology, including symmetry considerations [3], issues pertaining to DAS specifically [4], the relative merits of DAS vs. DOR and their advantages over prior techniques [5], and their application to solid-state materials [6, 7]. More recent reviews cover modern implementations of DAS and DOR in the context of how they 
compare to MAS techniques for quadrupolar nuclei $[8,9,10,11]$. In light of the quality and thoroughness of these previous contributions, DAS is discussed here primarily in the context of instrumentation development. The main focus of this review is SAS and VAS experiments for systems composed primarily of spin-1/2 nuclei and deuterium. Although VAS, SAS, and DAS experiments can be performed with essentially the same instumentation, the data analysis and scientific objectives of these experiments can be very different, as will be discussed in the following sections on the particular experiments and some applications. Belying their reputation as somewhat exotic techniques, all of the spatial reorientation experiments can be understood in the broader context of experiments that produce either dipolar recoupling or isotropic-anisotropic correlations, both of which are very common objectives in solid-state NMR. These methods have some important advantages and are worth revisiting given some of the recent trends in NMR spectroscopy, including extensive deuteration, fast sample spinning, and the convergence of solids and liquids methodology.

\subsection{Dipolar recoupling can be accomplished using pulse sequences, manipu- lation of the sample spinning, or modification of the sample itself.}

Dipolar couplings provide orientational restraints and information about local conformational mobility. In rigid solids, which are usually studied under MAS, homonuclear or heteronuclear dipolar couplings are frequently reintroduced using specialized pulse sequences. Recoupling sequences become more challenging to implement in samples where the dipolar interactions are partially averaged by random molecular motion, and more complex symmetrybased pulse sequences become advantageous. For soluble target molecules, residual dipolar couplings (RDCs) and residual CSAs can be induced using a variety of methods, including by mechanically aligning the macromolecule in a stretched gel $[12,13,14,15]$, or magnetically aligning it in a dilute solution of liquid crystals [16]. For membrane-associated peptides and proteins, additional alignment methods are available (reviewed in [17]), since the protein is associated with an orientable lipid bilayer. This weak-coupling regime can be accessed in solid samples by spinning only slightly off the magic angle $\left(1-3^{\circ}\right)$, heavily scaling the dipolar interactions. Particular spin systems can be selected using a combination of frequency-selective pulses and multiplequantum filtering [18]. The use of multiple-quantum filtering, without which it is difficult to separate dephasing due to weak couplings from incoherent 
signal decay, is an important detail. Even in this weak-coupling limit, dipolar couplings yield valuable orientational restraints. Dipolar couplings scale with internuclear distance as $1 / r^{3}$ for dilute spin systems, whereas NOEs and dipolar couplings in systems subject to spin diffusion scale as $1 / r^{6}$. In the case of many-spin systems such as protonated, uniformly ${ }^{13} \mathrm{C},{ }^{15} \mathrm{~N}$-labeled samples, magnetization exchange among ${ }^{1} \mathrm{H}[19]$ and ${ }^{13} \mathrm{C}[20]$ nuclei provide long-range distance restraints for structure determination.

Separated local field (SLF) experiments [21] are often used to determine structural parameters, molecular orientation, and segmental order parameters $[22,23]$. These experiments are attractive because of their versatility; the local interactions of the rare spins are selected, and the $2 \mathrm{D}$ versions can often be performed with natural-abundance rare spins. Incorporating polarization inversion and homonuclear decoupling in the SLF experiment (PISEMA) reduces linewidths by an order of magnitude, allowing dipolar coupling to be correlated with full chemical shift assignment [24, 25]. Many refinements have been added, including the use of symmetry principles to further narrow line widths in a pulse sequence for high-field experiments, as in SAMPI4 [26], and HIMSELF [27], as well as sensitivity enhancements effected by modified phase cycling and data collection schemes [28]. This general type of methodology has been widely used to obtain structural and chemical information about membrane proteins $[29,30]$. Orientational restraints can be determined using samples oriented between glass plates, or magnetically using the magnetic susceptibility anisotropy of the bicelles [31]. This line of pulse sequence development has led to a SLF-based assignment strategy where static, oriented membrane proteins are aligned parallel and perpendicular to the applied magnetic field [32], and an analogous version where the same type of data is collected on unorientated proteoliposome samples undergoing MAS [33]. In oriented sample NMR, correlated CSA patterns and dipolar couplings can be used to provide orientational restraints for structure determination even in the absence of isotropic chemical shifts [34].

Sophisticated hybrid approaches for membrane protein structure determination have been developed that use a combination of structural restraints collected using different NMR methods. One such method combines traditional solution NMR data from solubilized samples with dipolar couplings from PISEMA as well as a membrane insertion depth potential implemented in XPLOR-NIH [35]. The development of switched-angle spinning probes 
and methodology for oriented sample NMR potentially enables these and related techniques to be executed in a single experiment, without changing the sample composition and without removing the probe from the magnet.

\subsection{Reintroducing the CSA via a pulse sequence or changing the sample orientation provides structural information}

The chemical shift anisotropy (CSA) is a consequence of the non-uniform distribution of electron density in chemical bonds. In solid or oriented samples, the chemical shift has a sensitive dependence on molecular orientation, which may be partially or completely averaged by molecular motions. Thus, under conditions where each CSA pattern can be paired with an isotropic chemical shift for assignment purposes, fitting the CSA lineshape can provide beautifully detailed information about the orientation and mobility of particular chemical sites [36]. Despite its obvious utility, this becomes problematic in complex molecules containing many unique sites. When the spinning speed is less than the width of the anisotropic spectrum generated by the chemical shift anisotropy, spinning sidebands appear at multiples of the spinning speed, complicating the spectrum and reducing the effective signal-to-noise ratio, but allowing recovery of the CSA pattern using sideband analysis [37]. When the spinning rate is larger than the width of the pattern (in practice, this means that the sidebands have less than about $10 \%$ of the centerband intensity), direct analysis of a 1D spectrum is more difficult; the resulting spectrum has better resolution and reduced overlap between signals from different nuclei, but at the expense of losing the information contained in the sideband manifolds. Several sidebands are needed to determine the principal tensor values, with about 5 sidebands being sufficient to determine the anisotropy, and 6-10 for reliable determination of the asymmetry $(\eta)$ [38].

Numerous methods have been developed to overcome this limitation including the 1D TOSS (total suppression of sidebands) experiment [39], and refined versions that improve its performance at higher MAS rates and for samples with short $\mathrm{T}_{2}$ relaxation times $[40,41,42]$. In the PASS (phaseadjusted spinning sidebands) experiment, the phase of the sidebands is adjusted according to the sideband order [43]. Numerous other sequences for observing the CSA patterns under fast MAS have been developed.The chemical shift recoupling experiment of Tycko et al. [44] produces a CSA pattern in the indirect dimension corresponding to that of a well-resolved static spectrum, from which the principal tensor values can be straightforwardly read 
from the peak and shoulder positions of the CSA pattern. This experiment has given rise to several variants, including a version that is more tolerant of pulse-length errors [45] as well as a constant-time implementation [46]. Another approach correlates the isotropic (fast MAS) spectrum to an "amplified" CSA pattern mimicking that of a sample spinning at a fraction of the actual MAS rate [47]. Many other modern developments have focused on symmetry-based pulse sequences that exploit the different symmetry properties of the CSA and heteronuclear dipolar interaction $[48,49,50]$, not only allowing selective reintroduction of these effects, but also enabling determination of their relative tensor orientations [51]. Recoupling the CSA in the context of multidimensional experiments under fast MAS $[52,53]$ has led to the simultaneous determination of many chemical shift tensors in nanocrystalline proteins [54], and high-quality structures based on the associated orientational restraints [55]. CSA restraints have also been used to improve the quality of structures solved for membrane proteins in phosopholipid bilayers [56]. Introducing residual CSAs can also be accomplished by spinning the sample only slightly off the magic angle [57, 58], as has recently been exploited to investigate the membrane curvature induced by the influenza virus M2 protein [59].

For ease of spectral assignment and analysis, it is often convenient to observe the isotropic spectrum and the CSA in correlated Fourier dimensions. This has been accomplished using a series of rotor-synchronized $\pi$ pulses of alternating phases [60]. Other approaches include the 2D analogue of PASS, which uses a constant evolution time and simplified analysis [61]. Isotropicanisotropic correlations can also be obtained by manipulating the rotor speed. In magic angle hopping, (MAH) the sample hops among three discrete positions during the pulse sequence [62], at considerable sensitivity cost, since the sample is stationary during detection and thus has the expected short $\mathrm{T}_{2}$. It also collects the isotropic spectrum in the indirect dimension, which can cause problems with truncation if there are many isotropic sites to be resolved. The continuous rotation analogue of this sequence, magic angle turning (MAT) [63], uses rotor synchronized pulses to produce an isotropic spectrum in one dimension and a manifold of sidebands covering the full powder pattern in a second dimension. A major limitation of the original sequence was low sensitivity due to repeated projection pulses for storing the magnetization along the $z$-axis; this has been solved using the $5-\pi$ pulse version of MAT [64], and subsequent variations that optimize the data collection in the indi- 
rect dimension $[65,66]$. This approach can also be used to investigate other types of anisotropic broadening, such as magnetic susceptibility gradients [67]. Another variant of the 5- $\pi$ MAT sequence, but using moderately fast (up to $22 \mathrm{kHz}$ ) MAS rates has been optimized for disordered samples with large, potentially overlapping CSA manifolds [68]. In a more recent analysis, commonalities between the 5- $\pi$ MAT and PASS experiments have been exploited to generate a combined version with the simple data processing of PASS and the linear time increments in the indirect dimension afforded by MAT, making this experiment more convenient to implement [69]. Small CSA values can be measured under fast MAS using CSA scaling experiments, in which two identical sets of $\pi$ pulses are applied during an integer number of rotor periods, separated by a $t_{1}$ increment during which the magnetization is stored along $z[70,71]$. These methods have many attractive features; relatively large scaling factors on the order of $\mathrm{N}=10$ can be produced, and there are no isotropic shifts in the indirect dimension, allowing the experiment to be performed with a small number of $t_{1}$ points. Their major disadvantage is the loss of $50 \%$ of the signal due to the storage period. This issue can be alleviated in some cases by the 2D CSA-amplified PASS experiment, which is essentially a combination of 2D PASS and CSA amplification [72]. 2D CSAamplified PASS has a fixed experiment time, which minimizes phase artifacts due to transverse relaxation during the pulse sequence, and lacks a storage period, giving it the potential for higher sensitivity. Obtaining higher scaling factors requires more pulses applied over a longer time period, such that the sensitivity loss may exceed that of a conventional CSA amplification sequence with the same scaling factor. In practice, which of these approaches works better is likely to depend on experimental particulars including spectrometer performance and sample properties. Many sample reorientation experiments represent alternative approaches to obtaining this type of correlation spectrum, often using a simpler pulse sequence (although still requiring a storage period) and specialized instrumentation. 


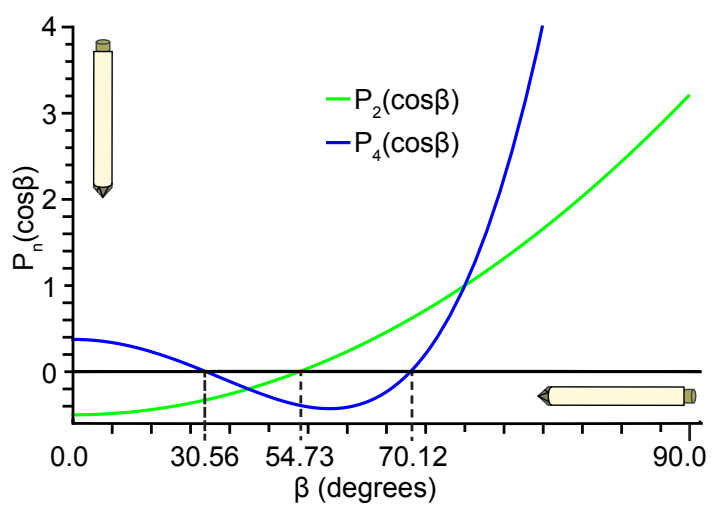

\section{Principles and experimental details of motional reorientation experiments}

\subsection{VAS, VACSY, and DACSY: experimental considerations and data anal- ysis}

In VAS, a series of one-dimensional experiments is collected, each at a different spinning angle. In MAS, second-rank interactions are averaged out over a full rotor period. When the sample is spun away from the magic angle at an angle $\beta$, anisotropic interactions are instead scaled according to the second order Legendre polynomial $P_{2}(\cos \beta)=\frac{1}{2}\left(3 \cos ^{2} \beta-1\right)$ (plotted in Figure 1), which has a value of zero at the magic angle. For systems subject to second-order quadrupolar broadening, an additional term that transforms as the fourth-rank Legendre polynomial, $P_{4}(\cos \beta)=\frac{1}{8}\left(35 \cos ^{4} \beta-30 \cos ^{2} \beta+3\right)$ (also plotted in Figure 1) becomes important. As is clear from the figure, the second-rank and fourth-rank terms do not vanish at the same angle. Therefore, an additional degree of freedom is required to average out both types of interactions. This is relevant to many types of chemical samples, because materials containing quadrupolar nuclei are often subject to both first-order and second-order quadrupolar broadening, or a combination of quadrupolar interactions, dipolar couplings, and chemical shift anisotropies.

These terms can be expressed in the lab frame using Wigner rotation matrix elements. The elements are functions of the rotor angle (as shown in Figure 2), so the choice of $\beta$ scales the terms. For the second-order Wigner elements, the $\mathrm{d}_{-1,0}^{2}$ and $\mathrm{d}_{1,0}^{2}$ terms dominate at the magic angle, though the $\mathrm{d}_{ \pm 2,0}^{2}$ terms also make a significant contribution. At $90^{\circ}$, the $\mathrm{d}_{-1,0}^{2}$ and $\mathrm{d}_{1,0}^{2}$ 

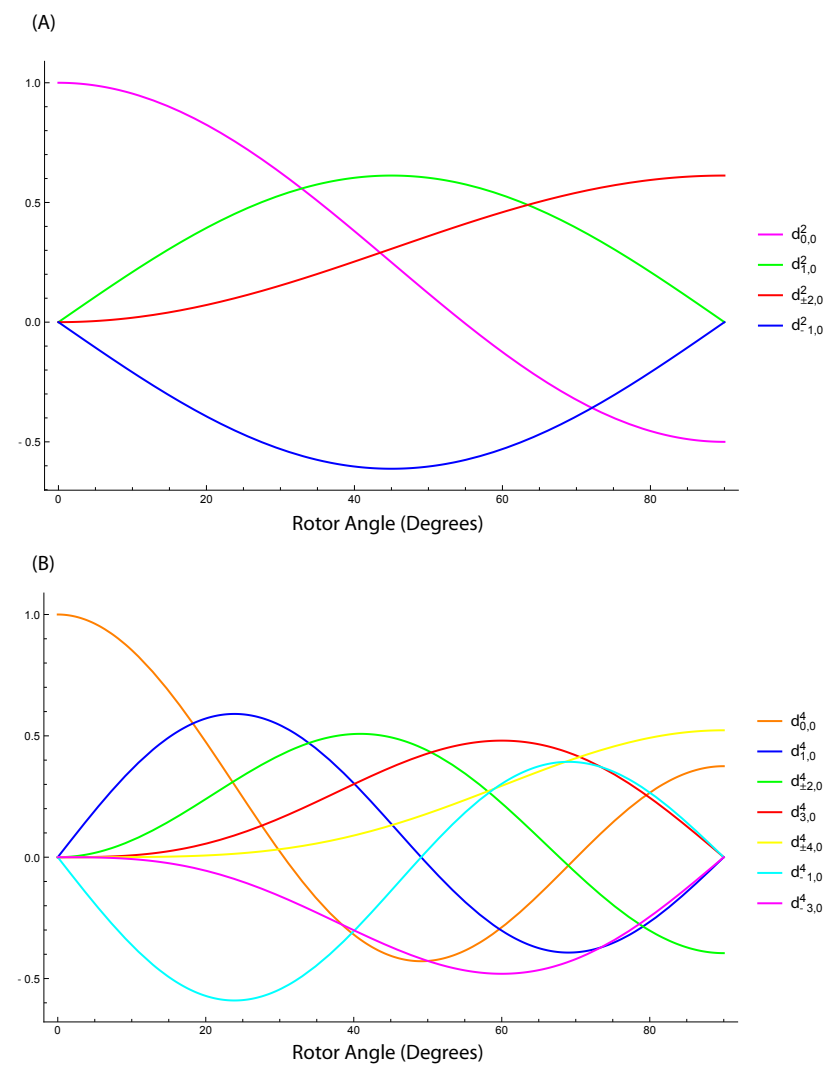

Figure 2:

Reduced Wigner matrix elements as a function of rotor angle (A) Second-order elements

Fourth-order elements [73]

terms vanish, leaving only the $\mathrm{d}_{0,0}^{2}$ and $\mathrm{d}_{ \pm 2,0}^{2}$ terms. Although the maximum theoretical CP transfer is not achieved away from $0^{\circ}$, it is easy to see that the intensity from $\mathrm{d}_{ \pm 2,0}^{2}$ at $90^{\circ}$ can be compared to that from the $\mathrm{d}_{-1,0}^{2}$ and $\mathrm{d}_{1,0}^{2}$ terms at the magic angle. For the fourth-order Wigner elements, the $\mathrm{d}_{0,0}^{4}$ element goes to 0 at the quadrupolar magic angles $30.56^{\circ}$ and $70.12^{\circ}$. The rest of the $\mathrm{d}^{4}$ elements are more involved. At $30.56^{\circ}$, the $\mathrm{d}_{-1,0}^{4}$ and $\mathrm{d}_{1,0}^{4}$ terms dominate, with the $\mathrm{d}_{ \pm 2,0}^{4}$ terms also making a significant contribution. At $70.12^{\circ}$, the $\mathrm{d}_{-3,0}^{4}$ and $\mathrm{d}_{3,0}^{4}$ terms make the greatest contributions, with the $\mathrm{d}_{-1,0}^{4}$ and $\mathrm{d}_{1,0}^{4}$ and also $\mathrm{d}_{ \pm 4,0}^{4}$ terms not far behind. The intensity of the $\mathrm{d}_{ \pm 4,0}^{4}$ terms is comparable to those of the dominant terms at both of the quadrupolar magic angles. CP match conditions, recoupling conditions, and other experimental parameters are greatly dependent on these Wigner elements. 
The analytical expressions for the positions and amplitudes for all the peaks in the multiplets are straightforwardly derived from the Hamiltonian for two coupled spins under VAS. Assuming that the (scaled) dipolar coupling is much smaller than the (scaled) chemical shift difference between the spins in the rotating frame chosen for analysis of the VAS experiment, the simplified Hamiltonian is similar to that for a two-spin system in isotropic solution, except for the orientation-dependent final term involving the dipolar coupling:

$$
\mathcal{H}=\mathcal{H}_{C S}+\mathcal{H}_{J}+\mathcal{H}_{D}
$$

where the components of the Hamiltonian are given by:

$$
\begin{aligned}
\mathcal{H}_{C S} & =\omega_{\alpha} \overrightarrow{I_{\alpha}} \cdot \stackrel{\leftrightarrow}{\sigma}^{P A F} \cdot \vec{B}_{0} \\
\mathcal{H}_{J} & =2 \pi J \vec{I}_{1} \cdot \vec{I}_{2} \\
\mathcal{H}_{D} & =-d\left(3 \hat{I}_{1 z} \hat{I}_{2 z}-\vec{I}_{1} \cdot \vec{I}_{2}\right)
\end{aligned}
$$

with the chemical shielding tensor in the principal axis frame defined as:

$$
\stackrel{\leftrightarrow}{\sigma}^{P A F}=\sigma_{i s o}\left(\begin{array}{ccc}
1 & 0 & 0 \\
0 & 1 & 0 \\
0 & 0 & 1
\end{array}\right)+\sigma_{z}\left(\begin{array}{ccc}
-\frac{1}{2}(1-\eta) & 0 & 0 \\
0 & -\frac{1}{2}(1+\eta) & 0 \\
0 & 0 & 1
\end{array}\right)
$$

and the homonuclear dipolar coupling constant as:

$$
d=\frac{\mu_{0} \hbar \gamma_{1} \gamma_{2}}{4 \pi}<r_{12}^{-3}>
$$

By considering the Hamiltonians as composed of tensors and spin operators,

$$
\mathcal{H}_{A}=\vec{I} \cdot \stackrel{\leftrightarrow}{A} \cdot \vec{S}_{A}=\sum_{\alpha, \beta=x, y, z} A_{\alpha \beta} \hat{R}_{\alpha \beta}
$$

it is then possible to construct spherical tensor operators from the Cartesian operators, obtaining Hamiltonians of the form:

$$
\mathcal{H}^{A}=\sum_{k=0}^{2} \sum_{q=-k}^{+k}(-1)^{q} \Lambda_{k-q}^{A} T_{k q}^{A}
$$


where the spherical tensor operators are defined as linear combinations of the Cartesian spin tensor components $\hat{R}_{\alpha \beta}$ [74]:

$$
\begin{aligned}
T_{00} & =-\frac{1}{\sqrt{3}}\left(\hat{R}_{x x}+\hat{R}_{y y}+\hat{R}_{z z}\right) \\
T_{10} & =-\frac{i}{\sqrt{2}}\left(\hat{R}_{x y}-\hat{R}_{y x}\right) \\
T_{1 \pm 1} & =-\frac{1}{2}\left[\hat{R}_{z x}-\hat{R}_{x z} \pm i\left(\hat{R}_{z y}-\hat{R}_{y z}\right)\right] \\
T_{20} & =\frac{1}{\sqrt{6}}\left(2 \hat{R}_{z z}-\hat{R}_{x x}-\hat{R}_{y y}\right) \\
T_{2 \pm 1} & =\mp \frac{1}{2}\left[\hat{R}_{x z}+\hat{R}_{z x} \pm i\left(\hat{R}_{y z}+\hat{R}_{z y}\right)\right] \\
T_{2 \pm 2} & =\frac{1}{2}\left[\hat{R}_{x x}-\hat{R}_{y y} \pm i\left(\hat{R}_{x y}+\hat{R}_{y x}\right)\right]
\end{aligned}
$$

and the spherical tensor components are defined as:

$$
\Lambda_{j k}=\operatorname{Tr}\left\{\stackrel{\leftrightarrow}{T}_{j k}^{\dagger} \stackrel{\leftrightarrow}{A}\right\}
$$

Thus, for this Hamiltonian: 


\begin{tabular}{|l|l|}
\hline \multicolumn{2}{|c|}{ chemical shift anisotropy } \\
\hline$T_{00}^{C S}=-\frac{1}{\sqrt{3}}\left(\hat{I}_{x} B_{0 x}+\hat{I}_{y} B_{0 y}+\hat{I}_{z} B_{0 z}\right)$ & $\Lambda_{00}^{C S}=-\frac{1}{\sqrt{3}}\left(\sigma_{11}+\sigma_{22}+\sigma_{33}\right)$ \\
$T_{20}^{C S}=\frac{1}{\sqrt{6}}\left(2 \hat{I}_{z} B_{0 z}-\hat{I}_{x} B_{0 x}-\hat{I}_{y} B_{0 y}\right)$ & $\Lambda_{20}^{C S}=\frac{1}{\sqrt{6}}\left(2 \sigma_{33}-\sigma_{11}-\sigma_{22}\right)$ \\
$T_{2 \pm 2}^{C S}=\frac{1}{2}\left(\hat{I}_{x} B_{0 x}-\hat{I}_{y} B_{0 y}\right)$ & $\Lambda_{2 \pm 2}^{C S}=\frac{1}{2}\left(\sigma_{11}-\sigma_{22}\right)$ \\
\hline \multicolumn{2}{|c}{ homonuclear $J$-coupling } \\
\hline$T_{00}^{J}=-\frac{1}{\sqrt{3}}\left(\vec{I}_{1} \cdot \vec{I}_{2}\right) \quad \Lambda_{00}^{J}=-\sqrt{3} J$ \\
$T_{20}^{J}=\frac{1}{\sqrt{6}}\left(3 \hat{I}_{1 z} \hat{I}_{2 z}-\vec{I}_{1} \cdot \vec{I}_{2}\right)$ & $\Lambda_{20}^{J}=0$ \\
$T_{2 \pm 2}^{J}=\frac{1}{2}\left(\hat{I}_{1 x} \hat{I}_{2 x}-\hat{I}_{1 y} \hat{I}_{2 y}\right)$ & $\Lambda_{2 \pm 2}^{J}=0$ \\
\hline \multicolumn{1}{|c}{ homonuclear dipolar coupling } \\
\hline$T_{00}^{D}=-\frac{1}{\sqrt{3}}\left(\vec{I}_{1} \cdot \vec{I}_{2}\right)$ & $\Lambda_{00}^{D}=0$ \\
$T_{20}^{D}=\frac{1}{\sqrt{6}}\left(3 \hat{I}_{1 z} \hat{I}_{2 z}-\vec{I}_{1} \cdot \vec{I}_{2}\right)$ & $\Lambda_{20}^{D}=\sqrt{6} d$ \\
$T_{2 \pm 2}^{D}=\frac{1}{2}\left(\hat{I}_{1 x} \hat{I}_{2 x}-\hat{I}_{1 y} \hat{I}_{2 y}\right)$ & $\Lambda_{2 \pm 2}^{D}=0$ \\
\hline
\end{tabular}

Having expressed the Hamiltonian in spherical tensor notation, the rotations necessary to move from the PAF reference frame through the rotor frame to the lab frame are easily expressed as functions of the Euler angles between reference frames and the Wigner rotation matrices. Defining the Euler angles from the PAF of spin 1 to the rotor frame as $\vec{\Omega}_{1}=\left(0, \theta_{1}, \phi_{1}\right)$ and from the PAF of spin 2 to the rotor frame as $\vec{\Omega}_{2}=\left(0, \theta_{2}, \phi_{2}\right)$ the Euler angles from the internuclear PAF to the rotor frame can then be defined as $\vec{\Omega}_{21}=\left(0, \theta_{2}-\theta_{1}, \phi_{2}-\phi 1\right)$. The spherical tensor components in the rotor reference frame can then be constructed with the Wigner rotation matrix 
elements:

$$
\Lambda_{k q}^{\prime}=\sum_{j=-2}^{(k)} D_{q j}^{2}(\vec{\Omega}) \Lambda_{k j}
$$

The transformation from the rotor frame to the lab frame can be achieved by considering the time-dependent Euler angles $\vec{\Omega}_{l f}=\left(-\omega_{R} t, \beta, 0\right)$, so that the new tensor components are given by:

$$
\Lambda_{k q}^{\prime \prime}=\sum_{j=-2}^{(k)} D_{q j}^{2}\left(\overrightarrow{\Omega_{l f}}\right) \Lambda_{k j}^{\prime}
$$

These results can be used to derive the frequencies and amplitudes for the 8 peaks in the coupled spectrum, the shapes of which are determined by the orientation dependence of the components in the lab frame. The shape of the dipolar term in the $F_{1}$ dimension is of particular interest; it is a scaled Pake doublet, the maxima of which are separated by the $J$-coupling value.

A VAS data set with a series of spinning angles contains a wealth of structural and orientational information, and the simplicity in instrumentation is offset somewhat by an increase in complexity of the data collection and processing. Because of the time-dependence introduced into the dipolar, CSA, and quadrupolar interactions during off-magic angle spinning, the values obtained from standard average Hamiltonian theory may not be sufficiently accurate to describe the VAS lineshapes broadened by CSA and quadrupolar interactions. Approaches to overcoming this challenge have included using stationary perturbation theory to calculate principal components of the quadrupolar and CSA tensors $[75,76]$ or using a set of homogeneous linear equations to simultaneously calculate all the sideband intensities resulting from CSA in spin 1/2 nuclei [77], and the quadrupolar interaction in halfinteger spin systems [78]. Such treatments have been productively applied to characterizing the local chemical environments of quadrupolar nuclei in complex materials, e.g. [79]. Variable Angle Correlation Spectrosopy (VACSY) is an alternative method in which many independent 1D spectra are stacked to produce an isotropic-anisotropic correlation [80] using an alternative sampling scheme, such as has long been common in the imaging context [81] and is gaining popularity in biomolecular solution-state NMR. 
A standard experiment for correlating unscaled, independent isotropic and anisotropic interactions uses a Cartesian sampling scheme, where spins evolve under an anisotropic interaction during the indirect dimension for a time $t_{a}$, followed by signal acquisition in the direct (isotropic) dimension as a function of an independent time parameter $t_{i}$. This sampling scheme, which is used for most multidimensional experiments, is shown in Figure 3a. In the course of a $2 \mathrm{D}$ experiment, $t_{a}$ is incremented and data collection is repeated for $n_{a}$ increments. The time-domain space $\left(t_{a}, t_{i}\right)$ is thus sampled in a rectangular grid, and a $2 \mathrm{D}$ Fourier transform of the signal $S\left(t_{a}, t_{i}\right)$ yields the $2 \mathrm{D}$ spectrum,

$$
I\left(\omega_{a}, \omega_{i}\right)=\iint S\left(t_{a}, t_{i}\right) e^{-i\left(\omega_{a} t_{a}+\omega_{i} t_{i}\right)} d t_{a} d t_{i}
$$

such that a slice though a peak in the $\omega_{a}$ dimension represents a powderpattern lineshape from which the principal components of the CSA can be extracted. The VACSY experiment uses a different, experimentally simpler strategy to sample the $\left(t_{a}, t_{i}\right)$ space, albeit a region of it constrained by the range of values available to the second-order Legendre polynomial, $-\frac{1}{2} \leq$ $P_{2}(\cos \beta) \leq 1$. Assuming that the rotor frequency is larger than the CSA, the precession frequency is approximately

$$
\omega \approx \omega_{i}+P_{2}(\cos \beta) \omega_{a}(\theta, \phi)
$$

where $\omega_{i}$ is the isotropic frequency and $\omega_{a}$, the anisotropic frequency, is determined by the CSA parameters $\Delta \omega$ and $\eta$, and the Euler angles $\theta$ and $\phi$ relating the PAS of each crystallite to the rotor frame. For each rotor angle $\beta$, the time-domain signal observed is

$$
S(\beta, t)=\iint I\left(\omega_{a}, \omega_{i}\right) e^{i\left[\omega_{a} P_{2}(\cos \beta) t+\omega_{i} t\right]} d \omega_{a} d \omega_{i}
$$

Because the spins evolve under the anisotropic interaction during time $t_{a}$ and the isotropic interaction during $t_{i}, t_{i}=\mathrm{t}$ and $t_{a}=P_{2}(\cos \beta) t$, and

$$
S\left(t_{a}, t_{i}\right)=\iint I\left(\omega_{a}, \omega_{i}\right) e^{i\left(\omega_{a} t_{a}+\omega_{i} t_{i}\right)} d \omega_{a} d \omega_{i}
$$

However, the region of $\left(t_{a}, t_{i}\right)$ space over which the above expression is integrated is set by the constraints on the value of $P_{2}(\cos \beta)$, covering a wedge in the space, as illustrated in Figure 3b. 


$$
I\left(\omega_{a}, \omega_{i}\right)=\int_{0}^{\infty} \int_{-t_{i} / 2}^{t_{i}} S\left(\omega_{a}, \omega_{i}\right) e^{i\left(\omega_{a} t_{a}+\omega_{i} t_{i}\right)} d t_{a} d t_{i}
$$

This process is analogous to changing the gradient values to sample a region of $k$-space in a projection-reconstruction imaging experiment. The sparsity of sampling in the grid space is determined by the number of spectra collected at different angles $\beta$. In the original VACSY experiment, a uniform sampling procedure was used within the wedge allowed by the scaling of $P_{2}(\cos \beta)$, and signals were interpolated between $\beta$ values to generate a dense array, allowing CSA powder patterns to be extracted. The principal components of the CSA tensors were thus measured for several model compounds with varying CSA values. Slow spinning (rotor frequency less than the magnitude of the CSA) does not appreciably degrade the performance of this experiment, since the principal tensor components can be recovered from the sideband patterns as in MAS and in other 2D isotropic-anisotropic correlations. 3D extensions of VACSY using either a series of 2D exchange experiments with VAS [82] or SAS [83], each collected at a different spinning angle, show chemical exchange between slowly interchanging conformers, as in a solid polymer.

Because nuclei with spin $>1 / 2$ are subject to interactions that transform as both second-rank and fourth-rank spatial tensors, their VAS spectra are more complicated, and extensions of VAS to quadrupolar nuclei can enable deconvolution of the isotropic chemical shift, the CSA, and the quadrupolar parameters for materials [75]. In a 3D DACSY experiment, a series of DAS experiments is performed, each with different complementary pairs of ( $\beta_{1}$, $\left.t_{1}\right),\left(\beta_{2}, t_{2}\right)$ values, and processed as a $3 \mathrm{D}$ data set [84]. This results in a set of anisotropic patterns providing a level of detail about the local chemical environment of each nucleus and the relative orientation between the CSA and quadrupolar tensors that is otherwise difficult to obtain without performing a single-crystal NMR experiment over a range of orientations. The data acquisition scheme for DACSY is illustrated in Figure 3c.

The VADOR experiment proposed by Amoreux combines some features of VAS and DOR for obtaining high-resolution spectra of quadrupolar nuclei [85]. The objective of VADOR is to obtain the true isotropic chemical shift values for nuclei where the resonance frequency is affected by the quadrupolar shift, under conditions where the outer rotor speed is fast enough to remove 

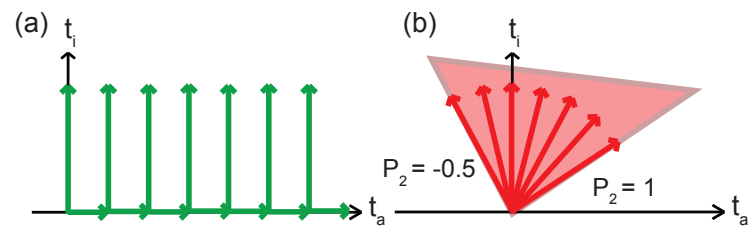

(c)

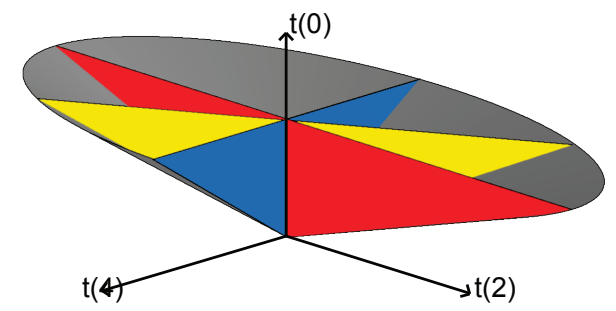

Figure 3: Data collection schemes for multidimensional isotropic/anisotropic correlations (a) Traditional Cartesian sampling scheme: the spin system evolves during the time $t_{a}$ under the effects of the anisotropic interactions. FIDs are then acquired as a function of the independent parameter time $t_{i}$ when the system evolves under the isotropic interaction [80]. (b) VACSY: the system evolves in the $\left(t_{a}, t_{i}\right)$ plane and the direction is changed within the two boundary values $\mathrm{P}_{2}(\cos \beta)=-0.5$ and $\mathrm{P}_{2}(\cos \beta)=1$, by changing the spinning axis angle $\beta$. The value of $\mathrm{P}_{2}(\cos \beta)$ cannot be made to go below -0.5 or above 1 no matter the spinning angle, limiting the coverage of the space [80]. (c) DACSY: Performing a series of 2D DAS acquisitions under various sets of complementary $\left(\beta_{1}, \beta_{2}\right)$ spinning angles creates a set of t-space cross sections. This set of cross sections then make up the 3D DACSY spectra used for quadrupolar nuclei [84].

all sidebands. Analytical expressions and simulated spectra were produced for two geometries, with the inner rotor spinning at $30.556^{\circ}$ and the outer rotor angle varying from a range of $0^{\circ}$ to $90^{\circ}$ for the collection of many $1 \mathrm{D}$ FIDs, which are then processed in a manner analogous to VACSY. An alternative geometry was also proposed for the case where CSA and/or dipolar couplings are non-negligible; in this case the angle of the inner rotor is $54.7^{\circ}$. At $30.556^{\circ}$, interactions that transform as $P_{2}(\cos \beta)$ are scaled by a factor of 0.6 , which is insufficient to simplify the spectrum if they are large. The DOR condition is satisfied with the inner rotor spinning at the magic angle and the outer one spinning at $70.1^{\circ}$. Although implementation of this experiment is very challenging from an instrumentation perspective, it has the potential to provide flexibility in obtaining useful spectral parameters for samples subject to strong quadrupolar interactions as well as dipolar couplings or CSA. 


\subsubsection{Dipolar couplings}

Recoupling of the dipolar coupling between two different rare spins (e.g. ${ }^{13} \mathrm{C}$ and ${ }^{15} \mathrm{~N}$ ) can be accomplished using rotational echo double resonance (REDOR), rotary resonance recoupling $\left(\mathrm{R}^{3}\right)$, symmetry-based sequences, and numerous variations for specialized purposes. Historically, all such pulse sequences based on a rotor-synchronized train of $\pi$-pulses were very susceptible to $\pi$-pulse imperfections, off-resonance effects, and fluctuations in the rotor speed. The spin echo, double resonance with flipped spinning (SEDORFS) was developed to circumvent some of these limitations [86]. SEDORFS is one of a class of experiments that provides simplicity in the pulse sequence at the cost of increased instrumental complexity; here, a single $\pi$ pulse on each heteronucleus per rotor period is used. The $\pi$ pulse on ${ }^{13} \mathrm{C}$, applied halfway through the dipolar evolution period, refocuses the carbon chemical shift. The ${ }^{15} \mathrm{~N}$ pulse then dephases the ${ }^{13} \mathrm{C}$ magnetization. In the REDORlike variant of this method, the carbon and nitrogen $\pi$-pulses are coincident. Experiments are performed in the presence and absence of these ${ }^{15} \mathrm{~N}$ dephasing pulses, with the ratio of these signals providing a measure of the ${ }^{13} \mathrm{C}$ ${ }^{15} \mathrm{~N}$ dipolar couplings. A SEDORFS experiment performed parallel to $\mathrm{B}_{0}$ is therefore equivalent to a static SEDOR experiment with evolution time $2 \tau$ in principle; however in practice the SEDORFS experiment was not performed at $0^{\circ}$ because it was done using a modified MAS probe with a solenoid coil. Another variation of this experiment is analogous to dipolar SAS, where the dipolar evolution period is incremented in multiples of the spinning speed, yielding a dipolar Pake pattern in the indirect dimension after a 2D Fourier transform. Interpretation of the SAS-like version is more straightforward, but its implementation requires that the spinning speed be greater than the width of the Pake pattern. Both types of SEDORFS experiment are most straightforwardly applied to systems where there is only a small number of chemically different observed spins, and where the dephasing spins have greatly different chemical shifts.

Many of the first motional reorientation experiments were performed on rigid solids at slow to moderate spinning rates, such that the heteronuclear dipolar interactions for protons directly bonded to ${ }^{13} \mathrm{C}$ or ${ }^{15} \mathrm{~N}$ were larger than the spinning frequency. In this case, the effects on polarization transfer from changing the spinning angle were negligible. In mobile solids and semisolids, where anisotropic interactions are partially averaged, the situation is 


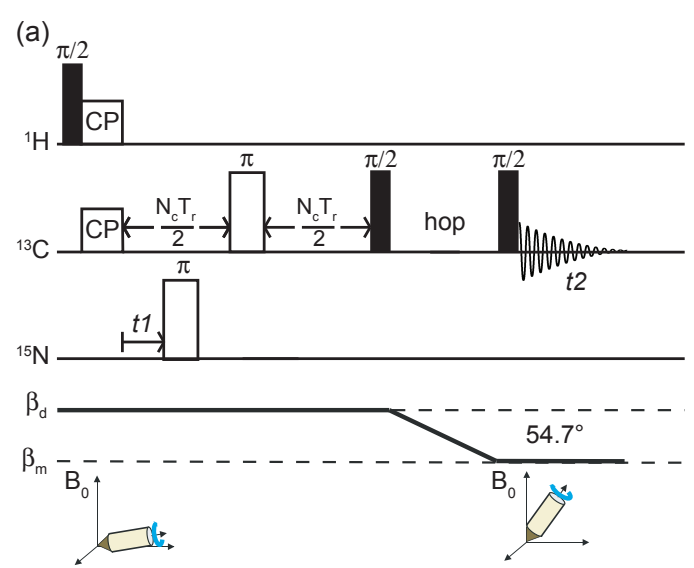

(b)

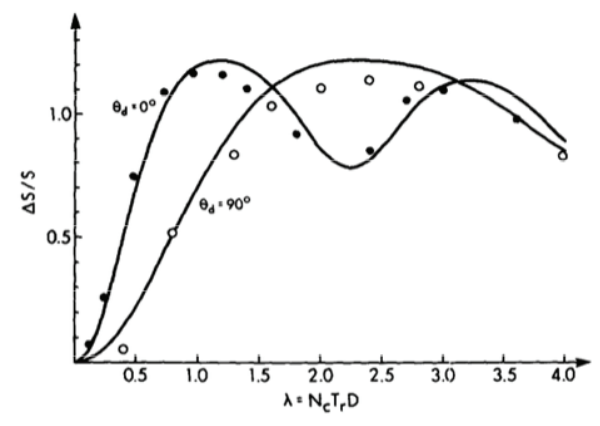

Figure 4: (a) The SEDORFS pulse sequence starts away from the magic angle. ${ }^{1} \mathrm{H}-{ }^{13} \mathrm{C}$ cross-polarization is followed by a dipolar evolution period with a $\pi$ pulse on ${ }^{13} \mathrm{C}$ to refocus isotropic chemical shift. The ${ }^{15} \mathrm{~N} \pi$-pulses induce dipolar dephasing. At the end of the evolution period, the magnetization is stored along the $z$-axis during the angle switch, and then read out at the magic angle. The experiment is performed with and without the ${ }^{15} \mathrm{~N}$ dephasing pulses, and the dipolar coupling value is obtained from the ratio of the signal obtained $\Delta \mathrm{S} / \mathrm{S}$, similar to the data acquisition scheme in REDOR. Figure adapted from [86] (b) Representative SEDORFS data for the model compound [L-4 ${ }^{13} \mathrm{C}$-, amide- $\left.{ }^{15} \mathrm{~N}\right]$ asparagine, showing the effect of the spinning angle on the dipolar couplings. Here the experiment was performed with the spinning angle during the evolution period set to $90^{\circ}$ (open circles). The SEDOR experiment (filled circles) is equivalent to SEDORFS at $0^{\circ}$, which was not directly performed because it was not possible in the probe used, which had a solenoid coil. The solid lines represent the corresponding theoretical curves. Figure reprinted from [86].

much more interesting. The partial decoupling induced by random molecular motions makes it possible to observe the characteristic time-varying Hamiltonians interacting in complex ways. Cross-polarization (CP), in which magnetization is transferred from abundant, high gyromagnetic-ratio nuclei to rare, low gyromagnetic-ratio nuclei [87], is the standard polarization-transfer method at low to moderate spinning rates. In the static case, $\mathrm{CP}$ occurs when the rf fields for two nuclei, $I$ and $S$, e.g. ${ }^{1} \mathrm{H}$ and ${ }^{13} \mathrm{C}$, are matched at the Hartmann-Hahn condition in the rotating frame. However, the experiment is most useful under magic angle spinning (MAS) [88]. It is well known that under MAS, interaction between the Hamiltonians for heteronuclear dipolar couplings and the sample spinning result in modulation of the CP intensity, 
which is experimentally observed as a sideband pattern in the $\mathrm{CP}$ match curve. Under MAS at a rotor frequency of $\nu_{r},\left|\nu_{S} \pm \nu_{1}\right|=n \cdot \nu_{r}$, where $\nu_{S}$ and $\nu_{I}$ are the rf fields applied to the $S$ and $I$ spins, respectively. Unlike in the static case, at the magic angle, the $n= \pm 1$ and $n= \pm 2$ conditions dominate the matching profile and the center band is mostly suppressed [89].

The truncated dipolar Hamiltonian for a spinning sample at an angle $\beta$ with respect to the magnetic field is the familiar expression $H_{D}=\Sigma_{i<j} \frac{1}{2} \gamma_{i} \gamma_{j} \hbar^{2}$ $\frac{1}{r_{i j}^{3}}\left(\mathbf{I}_{\mathbf{i}} \cdot \mathbf{I}_{\mathbf{j}}-3 I_{i z} I_{j z}\right)\left(3 \cos ^{2} \beta-1\right)$. When the sample is spun away from the magic angle, the angle $\theta$ between $r_{i j}$ and $B_{0}$ becomes time-dependent, with two periodic terms that depend on $\omega_{r}$ and $2 \omega_{r}$, producing sidebands at multiples of $\omega_{r}$ [90]. Both time-dependent terms have an average value of zero over a rotor period, yielding a scaled dipolar coupling, but their mutual interference affects any process that is mediated by the dipolar couplings. Taking some examples, when $\beta=0, \frac{1}{2}(3 \cos \beta-1)=1$ and the dipolar interaction is not scaled. When $\beta=54.7^{\circ}, \frac{1}{2}(3 \cos \beta-1)=0$, the magic angle. In the case where $\beta=90^{\circ}, \frac{1}{2}(3 \cos \beta-1)=\frac{1}{2}$. When $\beta=90^{\circ}$, the odd-numbered sidebands disappear because $\sin 2 \beta=0$.

The experimental consequences of this effect were first described by Sardashti and Maciel [91], who compared cross-polarization spectra under static conditions and with the sample spinning at the magic angle and at $90^{\circ}$ for both adamantane and a partially deuterated ${ }^{113} \mathrm{Cd}$ complex, ${ }^{1} \mathrm{H}\left(\mathrm{CO}_{2}\right)_{2} \mathrm{Cd}$. ${ }^{2} \mathrm{H}_{2} \mathrm{O}$. Although the $90^{\circ}$ spectra were collected in 'SAS' mode, where $\mathrm{CP}$ was performed off the magic angle while the signal was collected at $\beta_{m}$, this is still essentially a VAS experiment because the spectra obtained are onedimensional; detection at the magic angle simply ensures that the shims and sample position are uniform for all data sets. Both samples are characterized by very weak ${ }^{1} \mathrm{H}^{-1} \mathrm{H}$ homonuclear dipolar couplings, due to molecular motion in the case of adamantane and isotopic dilution in the case of the cadmium salt, making flip-flop transitions between these nuclei negligible. The effects therefore observed are primarily due to modulation of the ${ }^{1} \mathrm{H}_{-}{ }^{13} \mathrm{C}$ or ${ }^{1} \mathrm{H}-{ }^{113} \mathrm{Cd}$ heteronuclear dipolar interaction. In these samples, the static case produces the expected signal maximum at the Hartmann-Hahn condition, and the familiar sideband pattern at values of $n \pm \nu_{r}$ at the magic angle. At $90^{\circ}$, however, the $n= \pm 1$ conditions disappear and the modulation at $2 \nu_{r}$ dominates. The sidebands at $\pm 2 \nu_{r}$ and the centerband dominate the plot of 
signal intensity vs. ${ }^{13} \mathrm{C}$ or ${ }^{113} \mathrm{Cd} \mathrm{CP}$ field strength, and it is also noteworthy that the 0 and $n= \pm 2$ match conditions observed at $90^{\circ}$ are much broader than those observed for spinning at the magic angle $(n=0, n= \pm 1$ and $n= \pm 2$ ), because the ${ }^{1} \mathrm{H}^{1}{ }^{1} \mathrm{H}$ flip-flops are much more efficient when spinning at $90^{\circ}$. In the case where the ${ }^{1} \mathrm{H}-{ }^{1} \mathrm{H}$ homonuclear couplings are strong, as for polyethylene and ${ }^{1} \mathrm{H}\left(\mathrm{CO}_{2}\right)_{2} \mathrm{Cd} \cdot{ }^{1} \mathrm{H}_{2} \mathrm{O}$, their modulations of the heteronuclear interactions dominate, and the plot of signal intensity ${ }^{13} \mathrm{C}$ or ${ }^{113} \mathrm{Cd} \mathrm{CP}$ field strength reverts to resembling the static case.

More recently, $\mathrm{CP}$ match arrays have been performed as a function of spinning angle over the full range of angles between parallel to and perpendicular to $\mathrm{B}_{0}$, without modifying the probe or removing it from the magnet (Figure 5). Although the physical principles involved are well-known and described in the early MAS literature, this experimental verification is essential for developing next-generation VAS and SAS sequences because there is a trade-off between obtaining large enough couplings to provide effective distance constraints and avoiding power overload due to having to collect over a wide spectral width in the indirect dimension.

Adiabatic transfers have been shown to be effective for oriented samples, even at the magic angle [92], but for samples that are sensitive to rf heating or where $\mathrm{T}_{1 \rho}$ is short, a short $\mathrm{CP}$ period optimized to the spinning angle is more effective. The CP buildup curves were also measured as a function of spinning angle; the time dependence of the signal buildup was found to be different from the magic angle case described by $\mathrm{Wu}$ and Zilm [89]. At the magic angle the buildup of magnetization on ${ }^{13} \mathrm{C}$ occurs fastest at the $\mathrm{n}=$ 1 sideband condition, slightly more slowly at $\mathrm{n}=2$ and significantly more slowly at $n=0$. Similarly, on either side of the magic angle at $36^{\circ}$ and $66^{\circ}$ the magnetization is most intense and has the fastest buildup at the $\mathrm{n}=1$ condition, although the buildup is slower than at the magic angle in both cases. Comparing the behavior of the $n=0$ and $n=2$ conditions away from the magic angle reveals faster CP buildup at the $\mathrm{n}=0$ condition higher than the magic angle $\left(36^{\circ}\right)$ and at the $\mathrm{n}=2$ condition for lower than the magic angle $\left(67^{\circ}\right)$. At $36^{\circ}$, the difference between $n=2$ and $n=0$ is reduced. At $90^{\circ}$, the fastest buildup is obtained at the $\mathrm{n}=2$ condition. The match at this condition is easier to achieve experimentally for a mobile sample than those at $n= \pm 1$, because it is broader and therefore less sensitive to slight mis-setting of the rf field. 
$0^{\circ}$

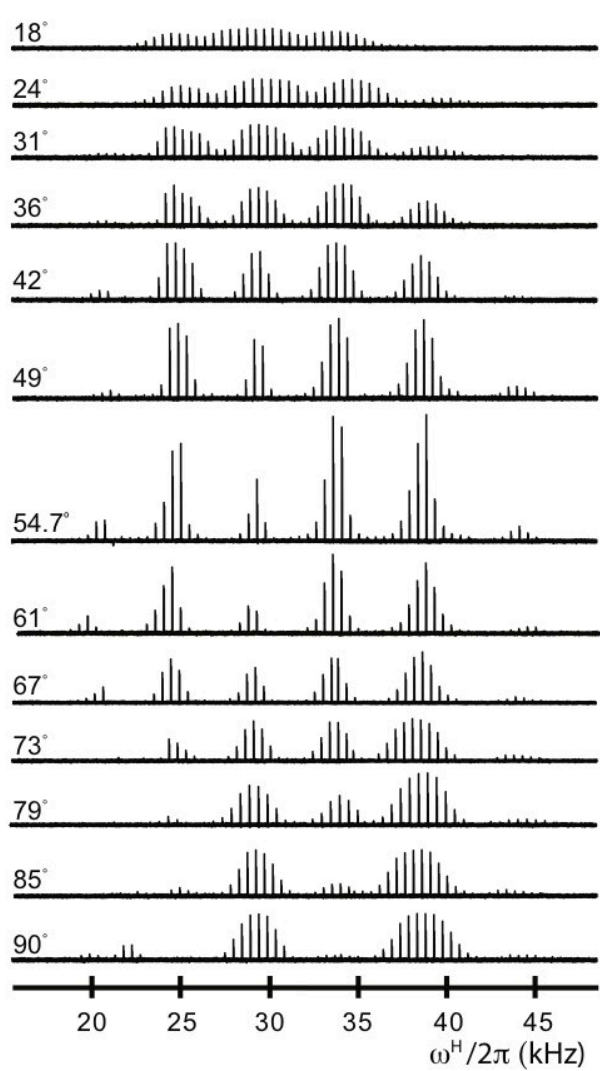

Figure 5: $\quad$ Experimental ${ }^{1} \mathrm{H}_{-}{ }^{13} \mathrm{C}$ crosspolarization arrays at different spinning angles. The ${ }^{13} \mathrm{C}$ rf field strength was held constant at $28 \mathrm{kHz}$ and the ${ }^{1} \mathrm{H}$ nutation frequency was arrayed from $15-55 \mathrm{kHz}$. The rotor frequency was $5 \mathrm{kHz}$ at all angles. The ${ }^{1} \mathrm{H} 90^{\circ}$ pulse length was $5.5 \mu s$, corresponding to a ${ }^{1} \mathrm{H}$ power $45.45 \mathrm{kHz}$. As expected, at the magic angle the $n=+2$ and $n= \pm 1$ sidebands give the strongest signals. The $n=-2$ sideband is weak because at this rotor frequency the efficiency of $\mathrm{CP}$ declines at $\mathrm{rf}$ amplitudes below $20 \mathrm{kHz}$. Above the magic angle the center band is more pronounced, and begins to dominate at small spinning angles, resembling the static case at $0^{\circ}$. The situation at $90^{\circ}$ is quite different - the $n= \pm 1$ conditions disappear and the match array is dominated by the $n=0$ and $n= \pm 2$ bands.

The efficiency of first-order recoupling sequences such as cross-polarization is in principle independent of the MAS rate [93], however, for some samples the sideband conditions become very narrow under fast MAS, making the technique difficult to implement in practice. This effect is particularly important in sparsely protonated spin systems and for molecules undergoing partial motional averaging of the dipolar interactions. In these situations, another strategy for generating isotropic-anisotropic correlations involves modifying the spinning during the evolution period. In the stop-and-go (STAG) experiment, the MAS is turned off during the $t_{1}$ evolution period by shutting off the drive air, which is controlled by a solenoid valve [94]. This method has many of the same limitations as SAS and MAH experiments because the magnetization must be stored along the $-z$-axis during a relatively slow mechanical process, in this case restarting the spinning. In all such techniques, the $T_{1}$ and 
relevant spin-diffusion processes for the detected nucleus must be slow relative to the mechanical step. Historically, such conditions have been relevant in the solid state only in plastic crystals or samples chosen for their sparsity of protons in order to observe these effects rather than due to any inherent interest in the sample. However, recent advances in solid-state NMR include extensive deuteration of biological macromolecules and very fast MAS, such that these conditions are now accessible for rigid solids as well. In the fast spinning regime, the zero-quantum and double quantum match conditions are better separated, and either can be used with low-power irradiation to provide frequency-selective CP [95]. Low-power cross-polarization can also be achieved at the $\mathrm{n}=0$ Hartmann-Hahn condition through second-order dipolar terms and J-coupling [96]. Improvements in pulse-sequence development and sample preparation methods that enable fast MAS are well worth exploring with modern VAS/SAS instrumentation as part of a diverse portfolio of experimental approaches. As in MAS, low-power decoupling can be used to mitigate potential sample heating issues and to work within the power limitations of low-inductance, low-E field proton coils. Many low-power ${ }^{1} \mathrm{H}-$ decoupling sequences have been developed for high-resolution multidimensional chemical-shift correlation spectra under fast MAS, e.g. [97, 98, 99].

\subsection{2. $C S A$}

The chemical shift anisotropy (CSA) contains useful information about chemical bonding, torsion angles, and many other aspects of molecular structure [36]. In relatively simple systems, the CSA lineshape can be resolved in a static spectrum and fitted to obtain chemical information. The HerzfeldBerger method is a refinement of this technique in which slow MAS is used, and the CSA pattern is recovered from the spinning sideband intensities [37]. Spinning at high frequencies, but off the magic angle also produces a scaled CSA pattern which is especially useful when $90^{\circ}>\beta>\beta_{m}$. This approach makes it possible to resolve break points in otherwise overlapping CSA signals [58]. This angular range in particular is useful because of the fact that the scaling factor applied to the CSA pattern ranges from 1 at $0^{\circ}$, parallel to $\mathrm{B}_{0}$, to 0 at the magic angle, to $-1 / 2$ at $90^{\circ}$. Thus, strongly overlapping signals can not only be scaled, but also flipped in sign by spinning closer to $90^{\circ}$ with respect to the static field, resulting in overlapping break points being moved to a less crowded region of the spectrum where they can be more easily resolved. This method of finding the principal components of 
the CSA tensor was demonstrated for polycrystalline samples of the model organic compounds 1,3,5 trimethoxybenzene and p-dimethoxybenzene, both of which were unresolvable by MAS alone at moderate MAS frequencies [57], and for cadmium complexes [100]. The methodology established in this proofof-principle study was then used to determine the principal components of the CSA tensors in substituted polycylic aromatic hydrocarbons (PAHs), which are difficult to study via NMR because of the long ${ }^{13} \mathrm{C}$ longitudinal relaxation times for unprotonated carbons. VAS was used to differentiate among carbons with similar isotropic values but different tensor components, making them difficult to resolve using MAS [101]. VAS enabled the highly overlapping CSA patterns of several aromatic sites to be resolved from each other and from the aliphatic signals, leading to some general heuristics for how the components of the CSA tensor are oriented relative to the aromatic ring and the $\sigma$ - and $\pi$-bonds. At the time, this class of compounds was interesting primarily as a model system for determining chemical shift tensor-structure relationships for studies of coal; they have more recently come under scrutiny as byproducts of cooking food, environmental pollutants arising from forest fires and anthropogenic combustion of biomass, and as model systems for the study of biopolymers such as melanin, giving new currency to methods for analyzing these challenging systems.

A combination of SAS and 2D exchange spectroscopy was used to correlate the ${ }^{13} \mathrm{C}$ chemical shift tensors to isotropic chemical shift values in the two magnetically inequivalent molecules found in the asymmetric unit of tropolone crystals [102]. In 2D exchange experiments, cross peaks represent spin exchange processes, i.e. a spin initially resonating at $\omega_{1}$ in the indirect dimension produces a resonance at $\omega_{2}$ in the direct dimension after a mixing time $\tau_{m}$ if there exists a mechanism for spin exchange. 3D-VACSY experiments based on a straightforward extension of 2D VACSY techniques were previously demonstrated for measuring chemical exchange processes. The extra evolution period is needed to correlate the magnetic shielding tensors under exchange [83]. However, this methodology is applicable only in the case where the spin exchange does not modulate the isotropic chemical shifts, i.e. where jumps between crystallographic sites or other self-diffusion processes are not accompanied by tautomeric rearrangements or proton shifts, and where spin diffusion processes are primarily between the same types of nuclei. These experiments do not correlate exchange processes between nuclei with different isotropic shifts. In the case of tropolone, proton shifts and double 
bond rearrangements are expected, complicating the problem of correlating the exchanging chemical shift tensors. The sample used for these experiments was labeled with $25 \%{ }^{13} \mathrm{C}$ at the carbonyl and hydroxyl carbons on different molecules. Earlier studies had found that at natural abundance the dominant exchange mechanism is chemical exchange, while at this level of enrichment it is spin diffusion. The types of spin exchange processes expected for tropolone include both molecular self-diffusion (jumps to crystallographically equivalent or inequivalent sites, possibly accompanied by proton transfer from the hydroxyl to the carbonyl carbon) and spin-diffusion (intra- or intermolecular, between equivalent or inequivalent sites). Because of the modulation of the isotropic frequencies $\omega_{i}$, yet another evolution period must be added to the VACSY exchange experiment, yielding a 4D experiment overall. Although this experiment is straightforward in principle, its implementation can be inconvenient due to the long experimental times needed. Recent advances in sparse sampling methodology $[103,104]$ could potentially make this experiment much more practical.

Alternatively, the full 4D correlation can be replaced by a selected series of 2D SAS experiments. This type of selective SAS exchange experiment was performed as a modification to the standard 2D exchange experiment, in which two angle-switching periods are inserted before the evolution and detection periods, during which $\beta$ is set to either the magic angle or $79.4^{\circ}$. In the specific case of tropolone as labeled for these experiments, only two types of observable spins are present, simplifying the data collection. Selective pulses were used to prepare magnetization on one type of spin, and then the appropriate combinations of frequencies and phase cycling were used to obtain the $2 \mathrm{D}$ correlation spectra. The pulse sequence starts with $\mathrm{CP}$ performed at the magic angle, followed by selection of the coherence on spin 1 or 2 , and an evolution period at $\beta=79.4^{\circ}$, where the value of $P_{2}(\cos \beta) \approx-0.5$. A 2D exchange experiment is then performed with detection at the magic angle. Before the detection period, the desired magnetization (site 1 or site 2 ) is selected. In this manner, the four correlation spectra required to investigate the correlations among spins 1 and 2 were collected. Standard SAS experiments were used to obtain the relevant chemical shift tensors. At long mixing times, the exchange spectra yield correlations between the chemical shift tensors of the exchanging sites, which provide information about the orientation of the chemical shift tensors in the molecular frame, given that the crystal structure is known. Comparisons among the $\omega_{1}-\omega_{1}, \omega_{2}-\omega_{2}, \omega_{1}-\omega_{2}$, and 
$\omega_{2}-\omega_{1}$ spectra revealed off-diagonal intensity in the 1-1 and 2-2 spectra only for long mixing times, indicating spin diffusion between the $1-1^{\prime}$ and $2-2^{\prime}$ sites, which are found in inequivalent molecules. The 1-2 and 2-1 spectra, which are related to each other by symmetry, likewise only show off-diagonal signal intensity at long mixing times. Two mechanisms contribute to the signals in this case; spin exchange between carbons 1 and $2\left(1^{\prime}\right.$ and $\left.2^{\prime}\right)$ and between 1 and $2^{\prime}\left(1^{\prime}\right.$ and 2$)$. Although the main exchange mechanism was spin diffusion in this case, this methodology is also applicable in the analogous situation where the exchange is dominated by jumps to crystallographically inequivalent sites, possibly accompanied by isomerization. In summary, this SAS version of the $2 \mathrm{D}$ exchange experiment provides a means of correlating two chemical shift tensors in the case where the isotropic shifts are modulated by the exchange.

VAS has been used to investigate the lineshapes of ${ }^{13} \mathrm{C}$ bound to ${ }^{14} \mathrm{~N}$, which are influenced by the ${ }^{13} \mathrm{C}-{ }^{14} \mathrm{~N}$ dipolar coupling, the chemical shift anisotropy, and the electric field gradient tensor of the ${ }^{14} \mathrm{~N}$. It has often been observed that the lineshape of a ${ }^{13} \mathrm{C}$ nucleus directly bonded to a ${ }^{14} \mathrm{~N}$ nucleus is broadened or split into an asymmetric doublet, even under conditions where the rest of the lines in the ${ }^{13} \mathrm{C}$ spectrum are narrow and symmetric. This effect results from incomplete alignment of the quantization axis of the ${ }^{14} \mathrm{~N}$ eigenstates with the external magnetic field. At low $\mathrm{B}_{0}$ values, the magnitude of quadrupolar interaction of the ${ }^{14} \mathrm{~N}$ is not large compared to that of its Zeeman interaction, leading to a condition where the quantization direction of the ${ }^{14} \mathrm{~N}$ eigenstates is not exactly directed along $\mathrm{B}_{0}$. In terms of the "dipolar alphabet", in addition to the usual A and B terms (the secular dipolar interaction) [105], it is necessary to also include the C and D terms (those depending on $\hat{I}_{z} \hat{S}_{x}$ and $\hat{I}_{z} \hat{S}_{y}$ ). The effects on the ${ }^{13} \mathrm{C}$ spectrum induced by the $\mathrm{C}$ and $\mathrm{D}$ terms do not average out under MAS. Depending on the desired experimental results, this effect is either a curiosity or a nuisance, since it is difficult to measure the CSA of such a ${ }^{13} \mathrm{C}$ without interference from the quadrupolar effect. Gan and Grant first made use of VAS to separate the CSA and dipolar couplings from the second-order quadrupolar effects in a study of several model compounds [73]. Using a magnetic field strength corresponding to a ${ }^{1} \mathrm{H}$ Larmor frequency of $100 \mathrm{MHz}\left(\mathrm{a}^{14} \mathrm{~N}\right.$ Larmor frequency of $7.3 \mathrm{MHz}$ ), and a rotor speed of $4 \mathrm{kHz}$, they compared spectra collected at the magic angle $\left(54.7^{\circ}\right)$ and the angle where $P_{4}(\cos \beta)=0(70.12$ ${ }^{\circ}$ or $\left.30.56^{\circ}\right)$. Comparison of these lineshapes and simulations thereof, using 
Hamiltonian treatment equivalent to first-order perturbation theory, enabled separation of the second order quadrupole effect from other spectral parameters, at least in the case where it is possible to determine the orientation of the EFG tensor from the MAS spectra. A follow-up study by Suits et al. used simulations of VAS experiments performed at much slower frequencies $(500-1100 \mathrm{~Hz})$ to determine the CSA tensor, the ${ }^{13} \mathrm{C}-{ }^{14} \mathrm{~N}$ dipolar couplings (and therefore the bond length), and the electric field gradient of the adjacent ${ }^{14} \mathrm{~N}$, all from 1D carbon spectra [106]. The sample used in this experiment was acetonitrile, which has a large CSA and a large C-N dipolar coupling due to its strong, short $\mathrm{C}-\mathrm{N}$ triple bond; under these conditions, a deviation on the order of $1-2^{\circ}$ from the magic angle produced sufficient differences in the spectra. Comparisons of ${ }^{13} \mathrm{C}-{ }^{15} \mathrm{~N}$ acetonitrile with ${ }^{13} \mathrm{C}$-enriched, natural abundance ${ }^{14} \mathrm{~N}$ acetonitrile revealed that this 1D VAS method provided accurate values for the bond length and the EFG, compared to measurements on static samples. However, the deviation of the CSA parameters from the measured static value exceeds the estimated experimental error, and the Euler angles describing the relative orientations of the CSA tensor and the ${ }^{14} \mathrm{~N}$ EFG were only measured to within about $10^{\circ}$.

\section{2. $S A S$}

\subsubsection{Magnetic susceptibility}

A different form of isotropic-ansisotropic correlation can be obtained using a variant of SAS called projected Magic Angle Spinning (p-MAS). In p-MAS, the full isotropic and anisotropic spectra are recorded using two different rotor angles, neither of which is the magic angle [107]. The objective of this experiment is to remove the constraint of having to choose the spinning angles based on the spin physics, opening up the possibility of using arbitrary angles determined by hardware constraints or those of sample geometry, e.g. in the case of spinning the magnetic field around a static sample. The two-dimensional version of this experiment yields scaled anisotropic spectra in the direct and indirect dimensions, with the scaled isotropic spectrum appearing along a projection, which is determined by the choice of angles. This experiment was demonstrated using glass beads and porous rock saturated with an oil-water mixture; in such samples anisotropic broadening due to magnetic susceptibility differences render the $1 \mathrm{D}$ spectra uninterpretable. The technique was also extended to the study of metabolites in liver and muscle tissue, where the linewidth is also dominated by magnetic susceptibility broadening [108]. A similar approach, based on VAS, was employed 
to separate the contributions of chemical shift and magnetic susceptibility differences to the separate signals observed for intracellular and extracellular water protons in suspensions of erythrocytes [109], with the conclusion that the chemical shift variations often observed in medical MRI are primarily due to magnetic susceptibility effects [110].

\subsubsection{Dipolar couplings}

In dipolar SAS, the indirect dimension is obtained off the magic angle, often parallel or perpendicular to $\mathrm{B}_{0}$, and the direct dimension is detected on the magic angle. This method can be used to measure internuclear distances in solids characterized by strong dipolar couplings $[111,112]$. The simplification of these complex spectra enables measurement of even very small dipolar couplings in strongly coupled systems, allowing observation of the fine structure in the powder pattern due to molecular motions and interactions with distant spins.

During $t_{1}$ the coherence (usually generated by an initial cross-polarization step, although other methods such as direct polarization or INEPT may also be used) is allowed to evolve under the influence of homonuclear dipolar couplings, often with a $\pi$-pulse introduced halfway through $t_{1}$ to refocus chemical shift. The $\pi$-pulse is usually not rotor-synchronized, so it introduces sidebands in the $\omega_{1}$ dimension. After the evolution period, a $\pi / 2$ pulse is applied to store the magnetization along the $z$-axis while the rotor axis is switched to the magic angle. Another $\pi / 2$ pulse then reads out the signal, and detection during $t_{2}$ occurs while spinning at the magic angle. Proton decoupling is employed throughout, except during the switching time. This experiment results in an isotropic-anisotropic correlation with an isotropic spectrum in $\omega_{1}$ and a Pake pattern in $\omega_{2}$. This method was used to determine ${ }^{13} \mathrm{C}-{ }^{13} \mathrm{C}$ distances in ${ }^{13} \mathrm{C}$-labeled zinc acetate and in a 14-residue peptide enriched with ${ }^{13} \mathrm{C}$ at two sites [111]. Distances between $2.4-4.2 \AA$ were observed. Although the dipolar Hamiltonians in these systems are truncated at first order (i.e. the relevant dipolar couplings are smaller than the chemical shift differences), complications arise in the peptide spectra because the dipolar couplings are comparable to the homogenous line width governed by the carbon $\mathrm{T}_{2}$ values. In this case, the dipolar couplings cannot be measured from singularities in the $\omega_{1}$ spectrum and must be obtained by fitting the Pake patterns and comparing them with the simulated spectra expected from 

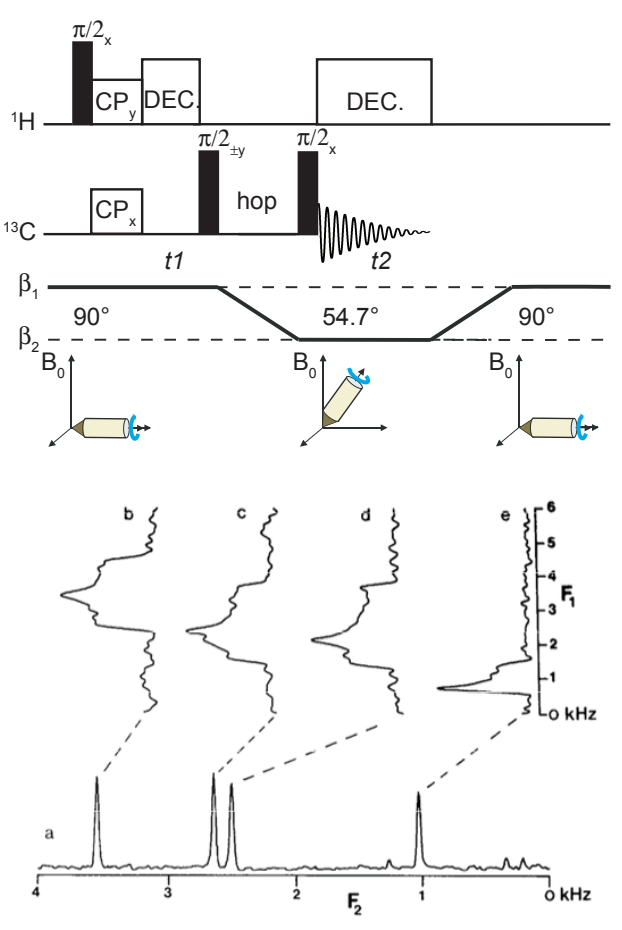

Figure 6: Top: SAS pulse sequence used for correlating the CSA powder pattern to the isotropic spectrum. Adapted from [113]. After a CP preparation period, magnetization evolves under the CSA at a spinning angle of $90^{\circ}$. The magnetization is then stored along the $z$-axis while the rotor hops to the magic angle for detection. Bottom: SAS NMR data showing the CSA patterns associated with particular sites in a ${ }^{13} \mathrm{C}$ SAS spectrum of polycrystalline p-methoxybenzene. (a) The isotropic spectrum, collected in F2 at the magic angle. (b)-(e) Slices from the $\mathrm{F} 1$ dimension for each isotropic carbon frequency. These powder patterns are half as wide relative to the static spectrum of this molecule because the rotor is spinning at $90^{\circ}$, where the value of $P_{2}(\cos \beta)$ is $-\frac{1}{2}$. Reprinted from [113].

different internuclear distances.

\subsection{3. $C S A$}

SAS approaches can also be used to correlate the isotropic spectrum to the CSA powder patterns, as first described by Bax et al. [113]. In this experiment, cross-polarization was performed perpendicular to $\mathrm{B}_{0}$, followed by the evolution period $t_{1}$. At the end of $t_{1}$, the spinning axis is switched to the magic angle, with the ${ }^{13} \mathrm{C}$ magnetization stored along $z$ during the switching time. Since the evolution period is performed at $90^{\circ}$, the CSA patterns are scaled down by a factor of 2 relative to the static spectrum. This can be an advantage because increasing the width of the $f_{1}$ dimension $(\approx 6.5 \mathrm{kHz}$ in this example) would require a longer evolution period, which reduces sensitivity and can be problematic for the probe and/or sample if high-power decoupling is needed. Representative data illustrating the correlation between CSA powder patterns and the easily-assigned isotropic spectrum are shown in Figure 6.

There is also a one-dimensional analogue of this experiment, in which ${ }^{1} \mathrm{H}$ - 
${ }^{13} \mathrm{C} \mathrm{CP}$ is followed by a rotor-synchronized DANTE sequence (or in principle, a selective pulse) at the magic angle, followed by switching and detection at $90^{\circ}$ [114]. The DANTE sequence places the magnetization corresponding to the selected isotropic ${ }^{13} \mathrm{C}$ chemical shift along the $z$-axis, and it is detected at $90^{\circ}$ after a dephasing period to remove residual transverse magnetization. CSA powder patterns are thus read out for every isotropic peak in individual 1D experiments rather than in two correlated Fourier dimensions. Because detection takes place at $90^{\circ}$, the CSA pattern is scaled by a factor of $-\frac{1}{2}$, although any angle could be used as long as the correct scaling factor, given by the value of $P_{2}(\cos \beta)$, is applied. As demonstrated by Maciel et al., finely ground powder samples must be used in order to obtain reliable values for the CSA tensors. Collections of larger crystallites can partially align themselves in the rotor, with some orientations favored and others disfavored, resulting in complex powder patterns [115]. This effect can produce experimental artifacts, but it also illustrates a useful property of oriented systems. This experiment can perhaps be considered as a precursor to the oriented sample SAS and VAS methodology later used to study liquid crystals.

\section{3. $M A F$}

Magic angle flipping experiments, where the rotor axis is hopped between $90^{\circ}$ and the magic angle, are also used to correlate isotropic and anisotropic spectra in inorganic materials. Even in cases where the ${ }^{29} \mathrm{Si}$ spectra are completely unresolvable in a 1D spectrum, they can be spectrally separated in a 2D MAF experiment based on the anisotropic lineshapes associated with chemical sites of different symmetry and coordination state. This approach has been used to identify and quantify the silicon species in alkali [116] and alkaline earth silicate glasses [117]. More recently, this method has provided information about the local environments of ${ }^{29} \mathrm{Si}$ sites in potassium disilicate [118] and magnesium silicate glasses [119]. Using the probe illustrated in Figure 7, the characteristic anisotropic line shapes for each type of environment were used to obtain quantitative data about the species populations. The principle components of the ${ }^{29} \mathrm{Si}$ nuclear shielding tensor anisotropy were also observed to be consistent with established trends. 


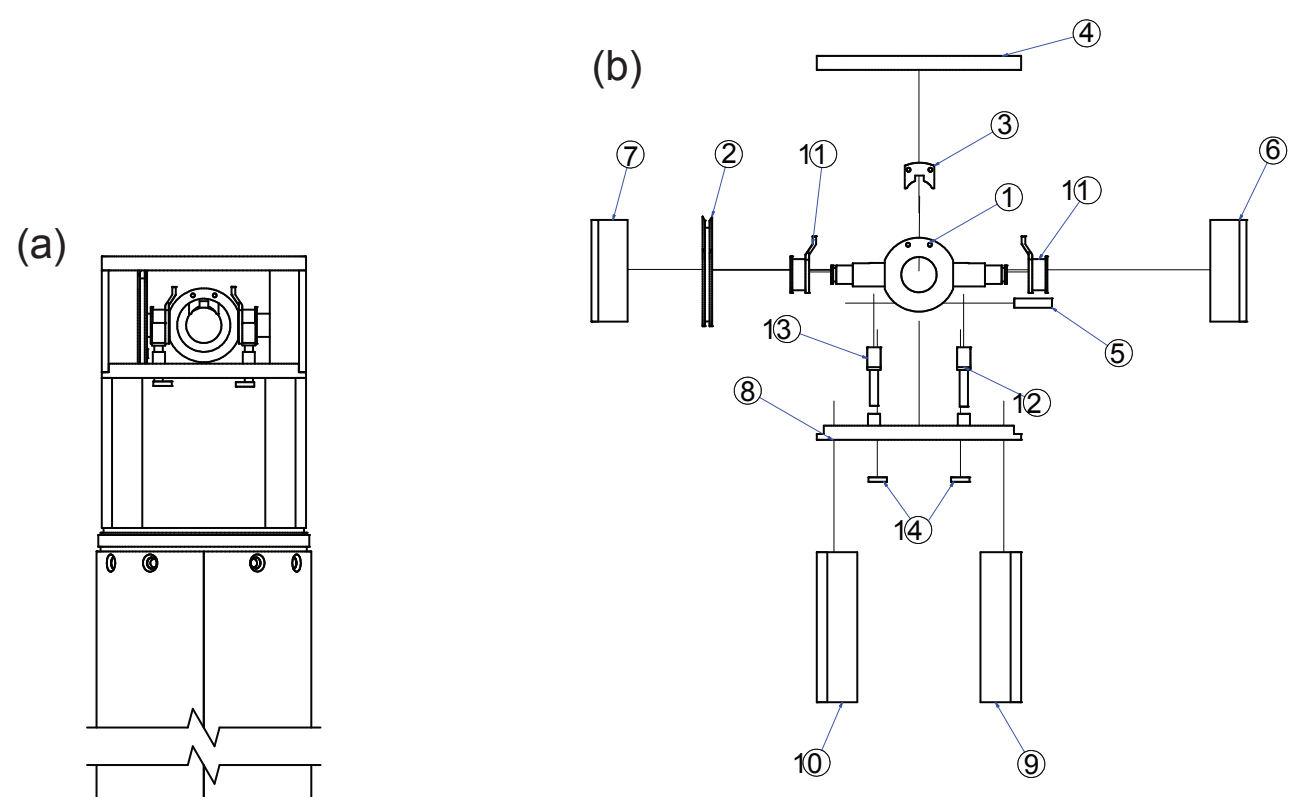

(c)

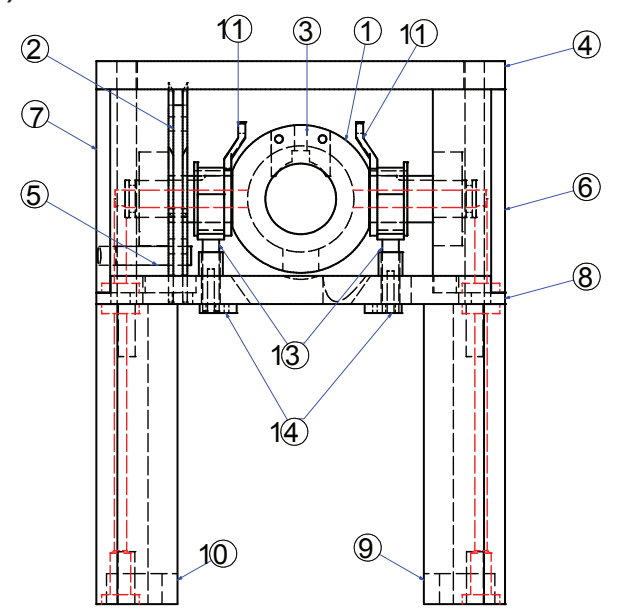

Figure 7: Magic-Angle Flipping probe: probe assembly (a) and the probe head in an exploded view to show all of the parts (b) and assembled (c) [bearing and drive air shafts highlighted in red]. Parts: 1) $4 \mathrm{~mm}$ stator housing, 2) probe head pulley, 3) $4 \mathrm{~mm}$ coil mount, 4) top ring, 5) pulley-stop, 6) stator housing post, 7) pulley-side stator housing post, 8) Head mount, 9 \& 10) stator housing post, 11) male slip ring, 12 \& 13) female slip rings, 14) thin film attachments. Drawings courtesy of Philip Grandinetti. 


\section{Instrumentation Development}

Spatial reorientation experiments have the reputation of requiring highly specialized instrumentation that is difficult to build or obtain. In reality, the instrumentation requirements of these experiments vary considerably, with some being very accessible to non-expert users and others in fact requiring purpose-built probes. VAS is the least instrumentally demanding of the spatial reorientation experiments because it is generally performed as a series of $1 \mathrm{D}$ experiments. Therefore, it does not require changing the spinning axis during a single experiment, obviating the requirement for fast switching and the signal loss associated with storing the magnetization along the $z$-axis. VAS can be performed using manual angle setting in an ordinary MAS probe, given a means of accurately measuring the spinning angle. On the other hand, SAS and DAS both require dedicated probes, which should ideally be optimized for the particular chemical systems under consideration.

Although they all share some common features, SAS/DAS probes are otherwise subject to the same design considerations and trade-offs as other solid-state and solution NMR probes; the desiderata for solids and oriented liquids are quite different and result in divergent design decisions. In the case of rigid solids having large quadrupolar interactions or strongly coupled dipolar networks, the most important factor is maximizing the rf field strength delivered for a given input power, either to excite the full bandwidth of quadrupolar nuclei that can have very broad spectra, or in the dipolar case, to provide efficient decoupling of strongly coupled heteronuclei. This is particularly important off the magic angle where dipolar interactions are less attenuated by the sample spinning. In this experimental regime, the samples are usually robust and typical experiment times are relatively short. Minimizing the switching speed is a critical factor because of $\mathrm{T}_{1}$ relaxation, spin diffusion, and relaxation of magnetization from the centerband to other energy levels in quadrupolar nuclei. For mobile solids or liquid crystals, decoupling is mostly accomplished by random motions of the molecules, and therefore considerations like rf homogeneity and shimming become more relevant.

Regardless of other considerations, the most important design feature of an SAS or DAS probe is the connection between the moving coil, which reorients on a millisecond timescale, to the stationary part of the circuit that 
interfaces with the NMR spectrometer. Because SAS and DAS experiments have at least two, and often three, Fourier dimensions, this connection must be able to withstand hundreds of thousands of angle flips without failing or compromising the rf stability. In addition to mechanical robustness of the angle switching mechanism, accuracy and reproducibility of the angle setting is needed in all cases in order to completely cancel the anisotropic interactions. A variety of SAS/DAS designs, both historical and currently used, represent different solutions to the problems presented by these experimental criteria.

Most SAS/VAS/DAS probes are based on established MAS designs, many of which use solenoid coils. Solenoids have many advantages, including their ease of construction, strong rf fields, and reasonably high rf homogeneity (with careful attention to spacing of the turns). They are also generally more efficient than the saddle coils more commonly found in liquids probes in terms of the $\mathrm{B}_{1}$ strength produced at a given power level, yielding higher sensitivity even though the effective rf field produced at the magic angle is scaled by a factor of $\sin 54.7^{\circ}=0.816$ [120]. However, solenoid coils have an important disadvantage in variable angle experiments; because the $B_{1}$ field oscillates in the direction along the coil axis, only the field component perpendicular to $B_{0}$ interacts with the spins. The $90^{\circ}$ pulse length at angle $\beta\left(p_{\beta}\right)$ is therefore related to the measured pulse width at $90^{\circ}, p_{90}$, by the expression $p_{\beta}=\frac{p_{90}}{\sin (\beta)}$. This is not a problem in an MAS probe, where the transverse component is still relatively strong, but in VAS or SAS, the $90^{\circ}$ pulse width becomes angle-dependent, such that the $90^{\circ}$ pulse with must be calibrated at each angle. Performance is therefore sharply degraded as the coil approaches the $B_{0}$ direction (illustrated in Figure 8). This situation is frustrating when it is desirable to measure the unscaled values of an anisoptric parameter such as the dipolar couplings or CSA. In such cases, transverse coil designs that provide a constant rf field perpendicular to $B_{0}$ regardless of the coil orientation are a better choice, ususally with some compromise in rf field strength or homogeneity. The split solenoid, which is illustrated in Figure 8, consists of a solenoid with a gap in the middle that is wide enough to admit the rotor, which is oriented perpendicular to the coil axis. This coil is easy to fabricate and produces a strong, orientation-independent rf field; however it suffers from reduced rf homogeneity and is therefore not ideal for experiments using many pulses.

Given these design constraints, two broad categories of coils have been 


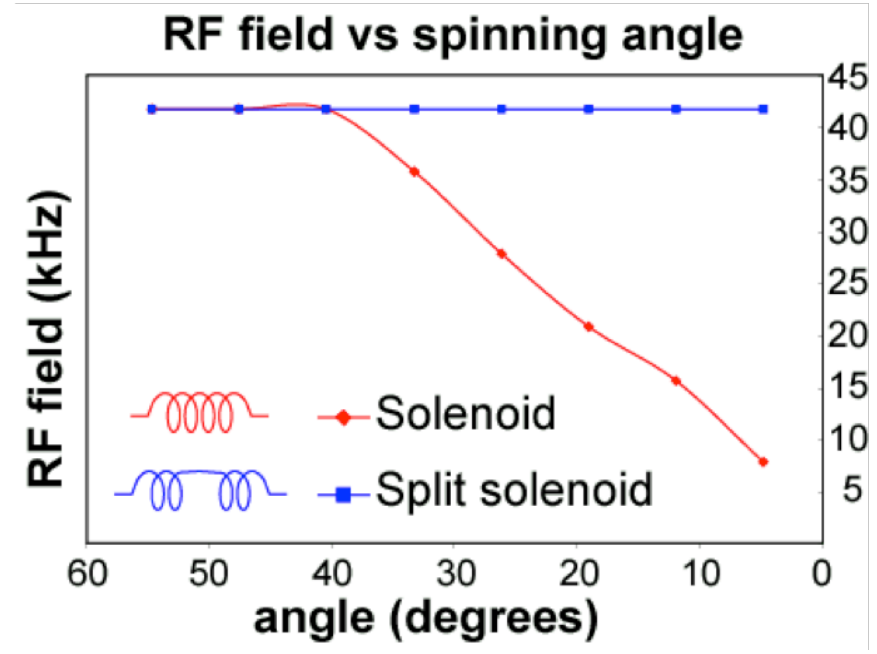

Figure 8: Measured $B_{1}$ field strengths for a solenoid and a split solenoid (an easily constructed transverse coil) as a function of the angle between the coil axis and $B_{0}$ (from [121]). Data were collected on adamantane using a $4 \mathrm{~mm}$ rotor in a sliding contact SAS probe similar to the one used in [84].

used for VAS/SAS/DAS probes: solenoid-like coils and transverse coils. Schematics of many of these coils are shown in Figure 9. Solenoid-like coils have the rf field along the long axis of the coil; however, many variations with different advantages have arisen. A variable pitch solenoid has a much better field homogeneity than that of an evenly-spaced solenoid of the same length and number of turns [122]. The solenoid can also be tilted such that the plane of each loop is not perpendicular to the coil axis [123], which also tilts the rf field it generates. Alonso, et al. have incorporated the Sun and Maciel design into their Double Helix Dipole coil [124], which consists of two coaxial tilted coils that together create an rf field orthogonal to $\mathrm{B}_{0}$. Other designs seek to reduce the electric field generated by an unbalanced solenoid, especially when the sample behaves as a lossy dielectric, as is the case for most hydrated crystals or precipitates of biological macromolecules. The scroll coil of Stringer et al. is much more homogeneous than a standard solenoid and also has a lower E-field, causing less rf heating in conductive samples [125]. Another low-E design is the Z-coil of Dillman et al., which is more homogeneous than either an evenly-spaced or a variable pitch solenoid [126]. On the other end of the spectrum are designs where the coil itself is solenoid-like, but the sample axis is perpendicular to that of the turns of the 
coil, providing a strong rf field at all angles, at the expense of homogeneity and/or sensitivity. A Helmholtz coil is a pair of coils placed at a distance equal to their radii. It is very homogeneous over a limited volume, though it suffers from a low filling factor and thus has limited efficiency. A split solenoid allows the rf field to be transverse to the sample axis, but has low homogeneity due to the shape of the field near the center of the sample.

The other category of coils, more commonly associated with solution-state NMR but finding greater applicability in solid-state probes as well, consists of those with an rf field transverse to the axis of the sample. A single-turn saddle coil has a good filling factor and reasonable homogeneity. A double or triple saddle coil has even better homogeneity than a single turn saddle coil, and also performs better at lower frequencies due to its higher inductance. Kentgens et al. have made use of this approach with a homebuilt DAS probe [127]. The rf circuit in their version uses a three-turn saddle coil with an inner diameter of $4.1 \mathrm{~mm}$ and sliding contacts with the leads colinear with the stator rotation axis. The rotor orientation is performed by a pulley system attached to a computer-controlled brushless servo motor.

A double saddle coil has been used in a capacitively coupled double-tuned VAS/ SAS probe for use with mobile solids and liquid crystals [128], with relatively good homogeneity. Some transverse coils are also designed to have a low electric field, such as the early exemplar of the genre, the AldermanGrant resonator [129]. Its low inductance design greatly reduces rf heating in conductive samples. A similar slotted tube resonator was used in a doubletuned capacitively coupled SAS probe for liquid crystalline samples. The low inductance of this type of coil, however, makes it only effective for detection of high $\gamma$ nuclei. The Modified Alderman-Grant Coil [130], with its capacitive bridge, has a low inductance. This makes it better for higher $\gamma$ nuclei.

Solenoid-like coils and transverse coils are frequently combined in static and MAS probes to create a coaxial pair of crossed coils. This type of design has often been used in static probes for oriented samples; a very successful recent version that can be used in both static and MAS probes is Gor'kov's $E$-free design [131] for a multi-channel probe. This geometry enables the use of a low electric field resonator for the high frequency channel and a solenoid capable of higher rf power for the other channels. Disadvantages include space constraints for the coaxial coils and reduced filling factor of the outer 
(a)

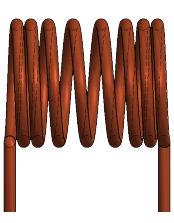

(e)

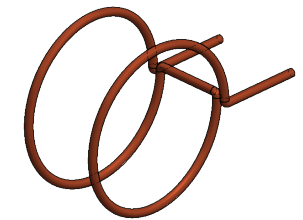

(i)

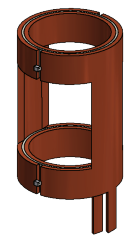

(b)

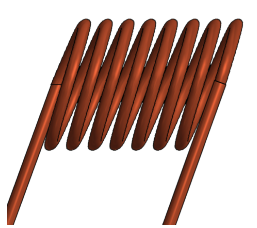

(f)

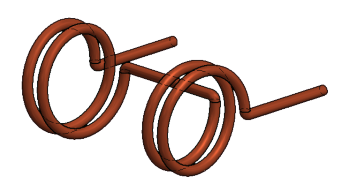

(j)

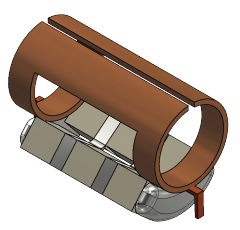

(c)

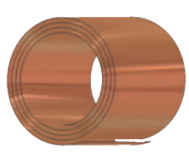

(g)

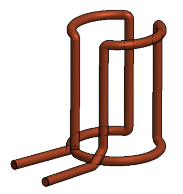

(k)

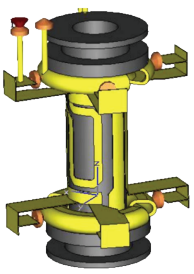

(d)

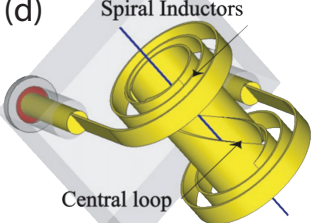

(h)

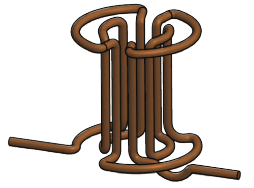

(I)

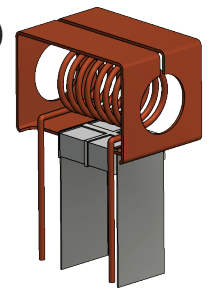

Figure 9: An NMR probe coil zoo showing both solenoid-like and transverse coils from the literature, all of which have the potential to be used in SAS/DAS probes depending on the application: (a) Variable pitch solenoid [122] (b) Tilted solenoid [123] (c) Scroll coil [125] (d) Z-coil, figure from [126] (e) Helmholtz coils (f) Split solenoid coil (g) Saddle coil (h) Double saddle coil [127, 128] (i) Alderman-Grant resonator [129] (j) Modified AldermanGrant resonator [130] (k) Doty's cross-coil design on a ceramic stator, figure from [132] (l) Gor'kov's crossed coil assembly [131]

coil. One method for dealing with this, while at the same time increasing the filling factor of the inner coil, is to make the inner coil out of copper foil and then wrap the coil around the stator with capacitors connecting the ends of the foil pattern [132]. This type of design has the potential to be used in SAS probes for deuterated solids and oriented liquids, for the same reasons it has gained popularity in MAS and static solids probes. 
(a)

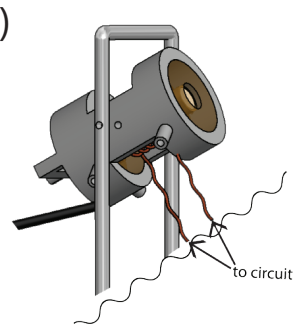

(b)

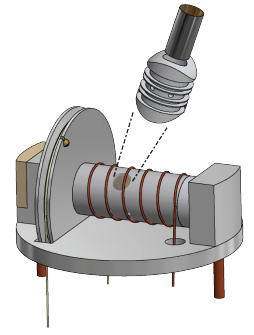

(c)

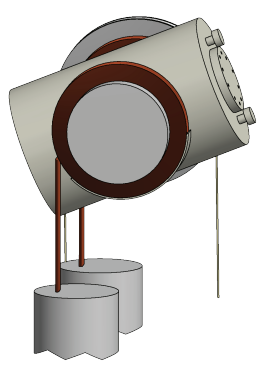

(d)

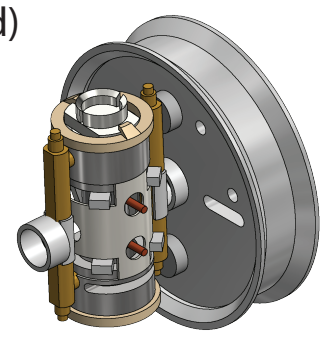

(e)

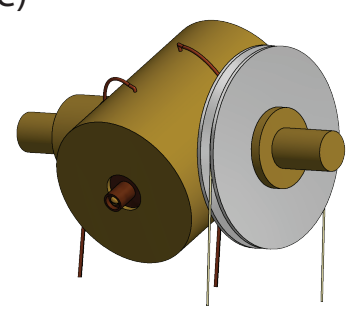

Figure 10: SAS/DAS probes for rigid solids: (a) Gerstein's DAS probe enhanced the spinning stability with the addition of another bearing air inlet at the bottom of the standard rotor design [133]. (b) Mueller's DAS probe delivers a consistent $\mathrm{B}_{1}$ field independently of the spinning angle by using a stationary rf coil wrapped around the entire stator assembly [134]. (c) Medek's DAS probe makes use of robust sliding contacts allowing for use over millions of rotor hops [84]. (d) Doty's HR-VAS probe utilized a cross-coil design to increase the sensitivity of the probe. It is also remarkable for its maximization of homogeneity and elimination of shimming artifacts from the stator assembly [135]. (e) Grandinetti's magic angle flipping (MAF) probe (a descendent of the probe described in [136]) has very precise control of the acceleration during angle switching [118].

Unlike in VAS, where the probe may be retuned at every angle, for all SAS/DAS probes, stability of the circuit tuning upon changing the angle is an important design consideration. This is illustrated in Figure 11 for a tuning-tube probe with sliding connections and a stepper motor, based on the design of Medek et al. [84]. A schematic of the angle-switching mechanism for this probe, which uses pulleys connected by stretch-resistant Spectra cord and a stepper motor mounted on a long actuator to minimize its exposure to the strong $B_{0}$ field, is shown in Figure 11a. The frequency response of the proton channel (500 $\mathrm{MHz}$ Larmor frequency) was measured using a Hameg frequency analyzer and is plotted in Figure 11b. The probe was initially tuned with the stator positioned at the magic angle, and the angle was then adjusted to $0^{\circ}$ and $90^{\circ}$ using the attached stepper motor, 
(a)

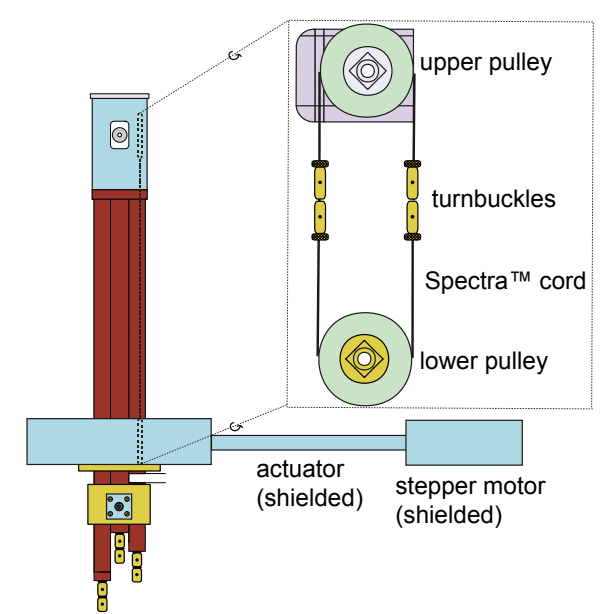

(b)

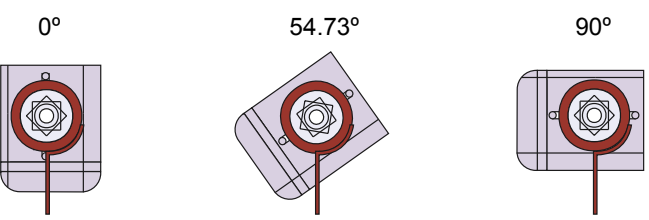

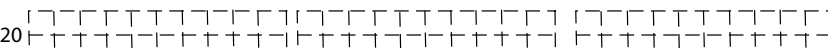

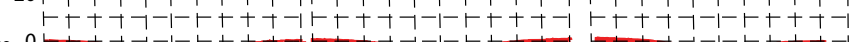

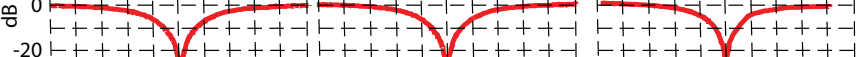

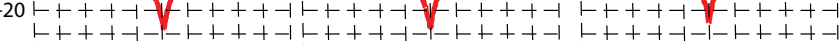

$-40\llcorner+++\dashv-1-++++-1 \leftarrow+++-1-1+++-1 \quad+++-1-1+++-1$

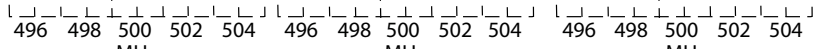

$\mathrm{MHz}$
Figure 11: (a) A schematic of one strategy for accomplishing angle switching in a DAS/SAS probe. The position of the stator is controlled using pulleys at the top and bottom of the probe assembly, connected to a stepper motor by way of a long brass actuator that keeps the stepper motor from coming too close to the magnet. (b) Tuning stability at a Larmor frequency of $500 \mathrm{MHz}$ for the probe depicted in part (a), measured using a Hameg frequency analyzer after tuning at the magic angle.

without retuning. It is possible to achieve sufficient tuning stability using sliding contacts, flexible leads, or capacitive coupling; however the moving connection is made, this is an important benchmark for a DAS/SAS probe.

\subsection{Rigid solids}

Many of the various coils used for other solid-state NMR experiments have also been used in VAS/SAS/DAS probes, with different strategies for managing the mobile connection points. A selection of DAS/SAS probes designed for rigid solids is shown in Figure 10. The first DAS probe described used a single-tuned solenoid coil wrapped around the spinning assembly, with a sliding contact between the leads emerging from the moving rf coil and the stationary leads attached to the matching network [137]. A refinement of this design used a double-tuned solenoid and beryllium-copper leads that maintain contact with the hopping coil by undergoing a "watch spring" motion [137]. In both of these designs, the $90^{\circ}$ pulse width depends on the 
coil orientation. As is clear from Figure 8 the rf field drops off rapidly as $\beta$ approaches $0^{\circ}$. In the particular case of using a solenoid coil with a relatively high filling factor at the angles required for DAS, this dependence does not present a major problem. For the configuration used in this paper, $p_{90}$ was about $3 \mu \mathrm{s}$, and $p_{\beta}$ was about $5.5 \mu \mathrm{s}$ at $\beta_{1}=37.38^{\circ}$ and $4 \mu \mathrm{s}$ at $\beta_{2}=79.19^{\circ}$. These correspond to rf field strengths of $83.3 \mathrm{kHz}$ and $45.5 \mathrm{kHz}$, respectively, which is sufficient for performing DAS experiments on quadrupolar nuclei with small to moderate quadrupolar linewidths. The leads described in this approach were reported to withstand about three million hops before requiring replacement, corresponding to several DAS experiments.

Further innovations building on this strategy have included more robust flexible leads, e.g. [138] and sliding contacts, e.g. [136].Two robust sliding contact DAS probes were developed in the 1990s, one based on the "watch spring" connection [136] and used for many pioneering DAS and SAS experiments and the other used by Medek et al. for DACSY [84]. The Medek probe used a multiply tuned circuit based on a Doty stator and the connection between the rotor and the stationary part of the circuit is made via rings soldered to either side of the coil sliding on beryllium-copper leads. This design was found to be robust over millions of rotor hops, which makes it well suited to perform long multidimensional experiments such as DACSY. In DACSY, several independent 2D DAS experiments, each with a few hundred scans for signal accumulation, are performed over the angle range of $18^{\circ}$ $\leq \beta_{1} \leq 39^{\circ}$ and $65^{\circ} \leq \beta_{2} \leq 90^{\circ}$. The probes first used for these experiments were equipped with 5 or $7 \mathrm{~mm}$ rotors, spinning at 5 or $10 \mathrm{kHz}$, respectively. The $90^{\circ}$ pulse widths were $1.7-4 \mu \mathrm{s}$, depending on the angle between the solenoid coil and $B_{0}$. The hopping time required was $45 \mathrm{~ms}$.

The Eastman et al. probe, which was a double-tuned single coil design, was similarly robust. This probe was used for a variety of applications, including DAS of quadrupolar materials and SAS for measuring internuclear distances [111] as well as investigating chemical exchange [102]. This design used a single solenoid made of copper ribbon wound on the outside of the stator, and connected to the stationary part of the circuit by beryllium copper strips that both bend and slide along the flattened wire of the coil. Because of its solenoid design, the $90^{\circ}$ pulse widths of this probe varied from 4-10 $\mu \mathrm{s}$, depending on the angle used. This probe used a Doty $7 \mathrm{~mm}$ MAS rotor system [139], and its mimimum switching time was 30-50 ms, depending on 
the angles chosen. More recent versions incorporating many of the positive features of this probe have been used for magic angle flipping experiments on solid materials, with smaller rotors, faster hopping times, and better $\mathrm{rf}$ efficiency $[118,119]$. Commercial implementations are available from both Doty Scientific and Revolution NMR.

Another type of single-coil, flexible lead design [133] makes use of highpower transmission lines to provide isolation in multiply resonant circuits [140]. Here the term "transmission line probe" refers to a circuit where the properties of transmission lines are used to provide rf isolation among the different channels [140], as opposed to a "tuning-tube probe," a different strategy in which transmssion line segments are used in lieu of traditional variable capacitors and inductors in order to increase power-handling capability and save space inside the magnet bore [141]. In the McKay transmission line type of design, the sample coil is located at the end of a large diameter transmission line having a length that is a multiple of the high-frequency $\lambda / 2$. This enables the tuning and matching elements for this channel (often traditional variable capacitors) to be placed outside the magnet bore. The rf for each lower-frequency channel is fed in through its own transmission line, each of which is connected to the high frequency line at a position corresponding to a node in the high-frequency standing wave. This kind of probe circuit has inherent isolation due to the positioning of this tap on the main transmission line. Multichannel VAS/SAS probes have been constructed using this strategy, taking advantage of its inherently high power handling and good isolation [86, 142].

Another solution to the problem of delivering a consistent $\mathrm{B}_{1}$ field independent of the spinning angle is to use a stationary rf coil surrounding the stator assembly, at the expense of filling factor and thus efficiency of the sample coil. A probe of this design was described and its performance demonstrated using ${ }^{2} \mathrm{H}$ VAS and ${ }^{17} \mathrm{O}$ and ${ }^{27} \mathrm{Al}$ DAS [143]. In this design, the stationary coil was a large solenoid oriented perpendicular to $\mathrm{B}_{0}$ and entirely outside the moving stator assembly. This approach provides a straightforward solution to the problem of connecting the electronics, given that it contains no moving connections, but it is likely impractical for most modern applications, where samples are precious and in short supply, requiring the filling factor to be maximized. 
SAS experiments can suffer from instabilities in the rotor spinning speed, particularly in the case of oriented sample NMR, where slow but very stable spinning speeds are desired. Many techniques for stabilizing MAS have been developed and can be applied to SAS as well. For example, buffering the air supply to the spinning controller by using multiple smaller ballast tanks in series rather than large ones can damp out pressure waves generated by the air compressor being turned on and off [144]. Typical spinning speed controllers consist of a feedback circuit that adjusts the flow of drive gas using a mechanical valve, in response to an optical signal [145]. In systems with optical spinning speed detection, painting multiple marks on the rotor such that the apparent spinning speed measured is a multiple of the actual spinning speed enables errors to be found and corrected by the automated spinning controller when they are smaller relative to the actual rotor frequency [146]. Very precise control of the spinning speed can also be achieved by varying the temperature of the drive gas [147], but this approach may be problematic in the case of liquid crystalline samples where small changes in temperature affect the phase behavior and alignment of the sample as well.

The paper by Mueller et al. [143] is notable for the careful attention given to minimizing the switching time and stabilizing the spinning. The switching is controlled by a stepper motor, which had precedent in earlier designs, e.g. [113], where a rotor spinning at $2.3 \mathrm{kHz}$ was switched and stabilized within less than one second. Mueller et al. very carefully analyzed the factors limiting the switching speed and the time required to stabilize the rotor. The authors determined that the minimum reorientation time for a change in angle on the order of $\pi / 4$, given optimal control of the switching system, is in the range of $1-4 \mathrm{~ms}$. In practice the limiting factor appears to be achieving precise enough control of the acceleration and deceleration of the stator during the switch. In the Mueller et al. design, switching was accomplished using a pulley system driven by a stepper motor, and switching (including rotor stabilization) required $28 \mathrm{~ms}$ to hop a $6.3 \mathrm{~mm}$ diameter rotor by $41.74^{\circ}$, the change in spinning angle required by a DAS experiment. Based on analysis of the torque generated by reorienting the rotor, rotor stability was found to be limited by turbulence in the bearing air flow, rather than by failure of the bearing air to support the extra load produced by the rotor during the hop.

The Gerstein SAS probe [133] was also notable for its fast switching with 
stable spinning: the angle can be changed by $45^{\circ}$ in $9 \mathrm{~ms}$ with spinning up to $10 \mathrm{kHz}$, with less than $1 \%$ variation in the spinning speed, although the stator assembly "rings" for $25 \mathrm{~ms}$. This probe used a modification of the spinning system designed by Wind et al. [148, 149], where an additional bearing air inlet was added at the bottom of the standard cylindrical rotor design. In the case where slow $(\leq 1 \mathrm{kHz})$, stable spinning is desired, spinning stability can also be enhanced by using drive tips with fewer fins and/or plugging some of the drive air inlet holes.

\subsection{Liquid crystals and semisolids}

VAS experiments on liquid crystals and membrane samples have a different history and purpose from those on rigid solids. Unlike in traditional MAS, the function of the spinning here is to align the director with the rotor axis. Averaging of anisotropic interactions is mostly performed by rotational diffusion, with an assist from rf decoupling. The spinning required for this application is quite slow compared to typical MAS rates, on the order of tens to hundreds of $\mathrm{Hz}$. Previous methods for aligning liquid crystals, including the use of electric fields and spinning the sample perpendicular to $B_{0}$ and slower than the critical speed, did not allow orienting the director close to the magic angle. The first VAS experiments on liquid crystals were performed using an electromagnet with a ${ }^{1} H$ Larmor frequency of $80 \mathrm{~Hz}[150,151]$. A stator assembly was constructed that was capable of spinning $10 \mathrm{~mm}$ samples tubes about any axis relative to $B_{0}$, allowing the director dynamics to be investigated over the full range of angles. Strong dipolar couplings, and therefore second order spectra, were observed when the sample was spun far from the magic angle. A rotor axis a few degrees off from the magic angle scaled the dipolar couplings, recovering the first-order spectra and enabling the determination of the signs of $J$ and $D$. This approach takes advantage of the mobility of the sample, making it an asset instead of a liability. Long-term, this method has the potential to enable RDC measurements and structure determination of membrane proteins, which do not assume their biologically relevant conformations in isotropic solution.

In contrast to the DAS/SAS probes built for experiments on solid materials, SAS probes optimized for studies of liquid crystals or semi-solid samples are subject to very different desiderata, other than accuracy and reproducibility of the angle setting, which are equally important in this context. The 
major considerations are rf homogeneity, spinning stability, temperature control, and robustness of the probe. Because the samples are partially mobile, rotational averaging narrows the line widths considerably, eliminating the need for high-power decoupling. Furthermore, the nuclei involved are usually not quadrupolar, aside from ${ }^{2} \mathrm{H}$, which has a relatively small quadrupolar interaction. For experiments on oriented membrane samples and other liquid crystals, the filling factor must be maximized to use the small samples available and the possibility of breakage or instability in the leads must be minimized during long experiments with many points in the indirect dimension. The increased stringency of these requirements is somewhat compensated for by the decreased necessity for high excitation or decoupling fields. Some examples of probes built for this purpose are shown in Figure 12.

In Variable Angle Spinning (VAS) of liquid crystals, the alignment is dominated by the shear forces produced by spinning; the molecules align parallel or perpendicular to the spinning axis depending on the sign of the magnetic susceptibility anisotropy. Changing the spinning axis scales the dipolar couplings $[155,156]$. An early VAS probe designed for studies of liquid crystals was based on a $10 \mathrm{~mm}$ liquids probe, with pneumatic spinning at speeds up to $900 \mathrm{~Hz}$ (Figure 12a). This probe achieved $3 \mathrm{~Hz}$ resolution in the ${ }^{1} \mathrm{H}$ spectrum at an operating frequency of $100 \mathrm{MHz}$, which represented a significant advance in resolution over previous designs, and enabled very precise measurements of ${ }^{1} J_{C H}$ and the $\mathrm{HCH}$ bond angle in methyl iodide. The angle setting was performed using a pinion angle adjustment mechanism controlled by a screw of precisely measured pitch, such that one turn of the screw corresponded to approximately $2^{\circ}$. The angle setting was measured optically, and was found to have excellent linearity over a broad range of angles [157]. Although this method of setting the angle is highly accurate and reasonably convenient, it has not come into widespread use. An updated version of a similar optical system for monitoring the spinning angle, with more details about its implementation and calibration, has been described by Mihaliuk and Gullion [158]. The first VAS and SAS experiments on bicelles established that their behavior is consistent with that of other lyotropic liquid crystals [159]. This work was performed using a homebuilt SAS probe with $4 \mathrm{~mm}$ rotors. This probe used a Helmholtz coil, making the $90^{\circ}$ pulse length independent of spinning angle. The angle switching was performed using a servo motor controlled by the pulse programmer, which produced a total switching time of $50 \mathrm{~ms}$, including $40 \mathrm{~ms}$ for the switching itself and $10 \mathrm{~ms}$ for the rotor 
(a)

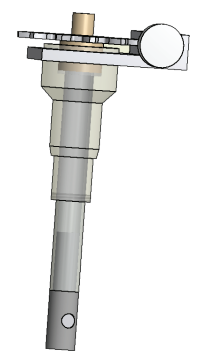

(c)

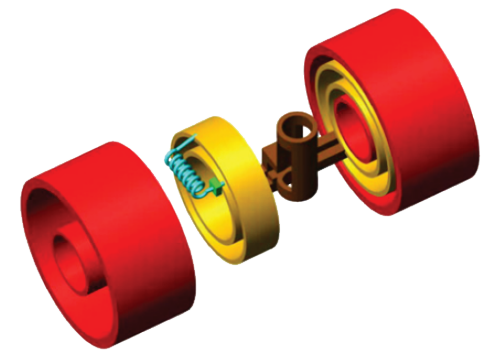

(b)

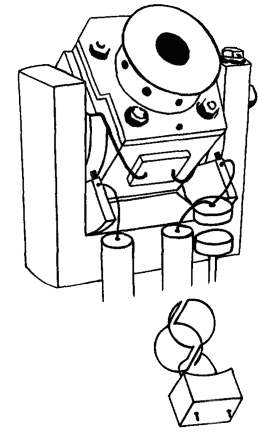

(d)

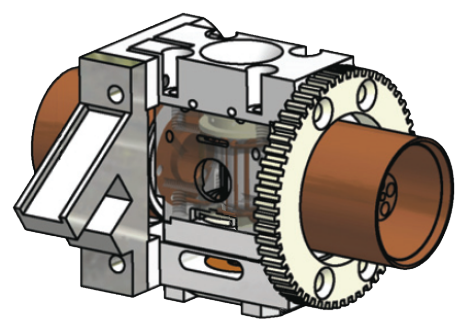

Figure 12: VAS/SAS probes for liquid crystals and semisolids: (a) The Väänänen et al. VAS probe provided high resolution and fine control of the spinning angle allowing for very precise measurements of the CH J-couplings [152]. (b) The Tomaselli et al. DAS probe uses sliding copper contacts and a pair of gold pantographs to transfer the rf pulses from the circuit to the transverse coil [153]. (c) The contactless resonator VAS/SAS probe utilizes capacitive coupling, eliminating the need for sliding contacts or flexible leads [154]. (d) Litvak's pneumatic SAS probe also makes use of capacitive coupling, but uses an airdriven switching mechanism instead of a stepper motor, making it possible to place the switching mechanism closer to the spinner module and reducing the hysteresis associated with the actuator [128]. Doty's HR-VAS probe (shown in Figure 10d in the solids probe section) can also be used for semisolids with only minor modifications [135]. 
spinning to stabilize.

Another approach to the problem of high-resolution VAS and SAS is the cross-coil HR-VAS probe of Doty and coworkers [135], (Fig. 10d) which uses flexible leads and a pulley-based switching mechanism. Cross-coil designs have often been used in NMR probes due to the absence of mutual coupling between orthogonal resonators and the ability to optimize the two coils for different frequency ranges, increasing the sensitivity of the probe [160]. The classic design utilizes a simple solenoid coil for the low-frequency channel(s) and a low-inductance resonator, such as that designed by Alderman and Grant, for proton decoupling [129]. Many variations have been described, with variations in the pitch and cross-section of the solenoid, and different types of slotted-tube resonators or saddle coils for the decoupling coil. Several modern versions have been built due to increasing interest in biological solidstate NMR, where samples act as lossy dielectrics and reduced electric fields are necessary to limit sample heating. These probes use a low-inductance coil for the proton channel, minimizing the voltage induced across the sample during high-power decoupling [132, 161, 162, 130, 131]. The HR-VAS probe is an early example of this type of probe, notable for its concinnity of design with respect to maximizing static homogeneity and eliminating sources of shimming artifacts due to the stator assembly and circuit elements near the moving coil. Rotor housing components are made cylindrically symmetric to the greatest extent possible, chip capacitors and exhaust holes are oriented at the magic angle with respect to the coil axis, and the effects on the magnetic field from the diamagnetic manifold rings defining the bearing and drive plenums are compensated by insertion of loops of paramagnetic wire. This design represents a major advance in HR-VAS experiments in terms of both rf homogeneity and flexibility of use for both solid and liquid crystalline samples.

Capacitive coupling is another approach to connecting the mobile and stationary parts of an SAS probe. Contactless resonator SAS probes have a mobile assembly built as a pre-tuned double resonant circuit, with the $\mathrm{rf}$ feeds for different channels (e.g. proton and carbon) coming in from opposite sides of the coil as shown in Figure 12b. The contactless resonator has no mechanical contacts between the coil and the rest of the circuit. The sliding surface is Teflon, which eliminates stability problems due to mechanical aging of sliding contacts and also minimizes stray reactances during reorien- 
tation [154]. This design also has the advantage of being balanced on both channels, obviating some of the problems that can occur with unbalanced circuits at higher field strengths, such as mismatch of the rf profiles of different channels [163], rf-induced thermal gradients, and arcing of the coil at the high voltage points at either end [164]. The sample coil itself is a modified version of the slotted tube resonator originally developed by Alderman and Grant to reduce heating due to rf irradiation of conductive samples. It has the added advantage of providing a homogeneous, orientation-independent field. Because it is a single-loop coil with inherently low inductance, this type of resonator performs best at higher frequencies. This coil showed good ${ }^{1} \mathrm{H}$-channel performance and excellent RF homogeneity on both channels, but poor ${ }^{13} \mathrm{C}$ sensitivity, indicating that a higher inductance is necessary for carbon-detected experiments [121].

The second-generation contactless resonator probe addressed two outstanding issues: the need for better performance on the carbon channel and a faster switching mechanism [128] (Figure 12d). The required higher inductance is provided by a double saddle coil, which is pictured in Figure 9(h). This coil was chosen as a compromise between low frequency and high frequency performance. This probe was designed to deliver a constant rf field strength independent of spinning angle to allow for maximum flexibility in testing sample preparations and experiments for SAS in oriented systems. Because a self-supporting wire gives poor field quality and is prone to misalignment that impairs spinning, the top and bottom of the coil are supported by machined Kel-F tablets. The inner diameter of the ceramic piece must provide enough room for free rotor spinning. In MAS probes, the Hall sensor is usually mounted directly on the stator [165] and calibrated by recording the voltage when the stator is at the magic angle, measured by maximizing the sidebands of $\mathrm{KBr}$ [166]. In an SAS probe, the sensor cannot be mounted on the spinner module during an experiment. Thus, the sensor is attached to the stator by a removable bracket and calibrated, with the Hall voltage recorded for a range of angles. Angle switching in most SAS probes has been performed using a stepper motor or servo motor. This provides great flexibility of control over the motion of the spinner; however, the necessity of locating the magnetic motor far enough from the superconducting magnet leads to slower switching and possible failure of the motor. Using pneumatics [167] it is possible to place the switching mechanism close to the spinner module. Solenoid valves replace the motor, and the motion is actuated using 
pressurized air.

In the Litvak SAS probe, the angle switching is performed by a steam engine-type pneumatic mechanism with a geared connection between the piston and the spinner module. The cylinder is mounted between the base plate and the middle plate of the probe body. The vertical position of the cylinder is adjusted through a worm drive. The piston travel is set between the bottom of the cylinder and a separate brake disk, which moves inside the cylinder. It is connected to a threaded rod outside the cylinder through a clip. To set $\beta_{1}$ (smaller) and $\beta_{2}$ (larger), one adjusts the vertical position of the pneumatic cylinder and of the brake disk, respectively. Pneumatic switching is achieved by alternately applying pressure and vacuum. Linear motion of the piston is converted into the angle switching of the spinner through a rack-and-pinion gear. The angle switch time depends on the pressure applied, with higher pressure resulting in faster switching. Figure 13 shows the time-domain signal of $\mathrm{KBr}$ for different values of the switching delay. With $t=0$ set when the stator begins to move $(12 \mathrm{~ms}$ from the closing of the valve), it takes $14-16 \mathrm{~ms}$ for the stator to reach the magic angle and an additional $1 \mathrm{~ms}$ for the spinning to fully stabilize. The minimum time required for the angle switching with 75 psi input pressure is thus $17 \mathrm{~ms}$, including the time for the spinning to stabilize. Given that the ${ }^{1} \mathrm{H}$ longitudinal relaxation time $T_{1}$ is on the order of a second for a typical hydrated solid protein sample, this is sufficient for performing the desired experiments. Furthermore, this is the time required for switching from $0^{\circ}$ to the magic angle; in order to obtain manageable coupling values, real experiments are likely to require a smaller change in angle. Regardless of how the angle setting and swtiching are accomplished, the hopping time, including the time it takes for the spinning speed to stabilize, is a key DAS/SAS probe benchmark that should be optimized.

\section{Applications: Inorganic Materials}

The study of inorganic materials was the impetus for the development of DAS and Double Rotation (DOR), the earliest and best-known of the spatial reorientation experiments; together they represent the first methods available for obtaining high-resolution spectra of nuclei subject to broad- 


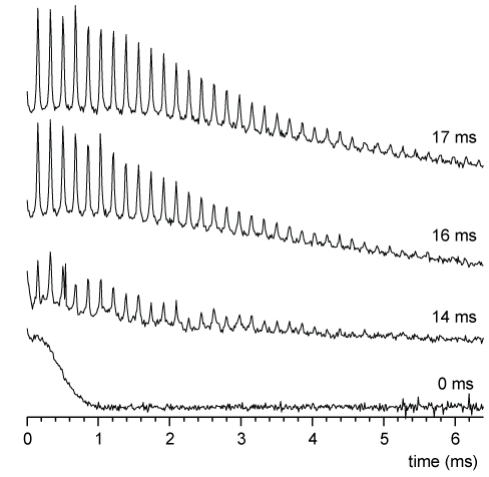

Figure 13: Time-domain ${ }^{79} \mathrm{Br}$ signals of $\mathrm{KBr}$ as a function of switching time. Here $t=0$ is counted as the time when the stator begins to move, $12 \mathrm{~ms}$ after the TTL pulse signaling the closure of the valve. No rotational echoes can be seen at $t=0$, as the sample is spinning at $0^{\circ} .14$ ms later, the sample is moving toward the magic angle. At $16 \mathrm{~ms}$, the sample has reached the magic angle, but takes an additional ms to fully stabilize, as can be seen by comparing the small rotational echoes near the end of the acquisition time. 16 scans were taken to confirm that the measurement is reproducible.

ening from the second-order quadrupolar interaction [168]. In DAS, first introduced by Pines and coworkers, the sample is spun about two different angles sequentially, averaging the second-order quadrupolar tensor, but not homonuclear dipolar couplings [137, 169]. With appropriate timing of the evolution periods, DAS can be performed at various sets of complementary angles, resulting in different sideband patterns [170]. DOR, on the other hand, can also effectively average homonuclear dipolar couplings [171]. In DOR, first proposed by Llor and Virlet [172] and implemented by Samoson, Pines and co-workers $[173,174]$, the sample is housed inside an inner rotor spinning at either $30.6^{\circ}$ or $70.1^{\circ}$, which is itself inside an outer rotor spinning at the magic angle; thus both the first- and second-order quadrupolar interactions are averaged out. The very slow spinning frequencies accessible to the outer rotor were a major limitation of the early DOR probes. Samoson et al. made this methodology more practical by improving the spinning stability and enabling the outer rotor to spin at faster rates $[175,176]$. In addition to their utility in studies of half-integer quadrupolar nuclei, DAS and DOR can also be used to remove second-order broadening from overtone spectra of spin-1 nuclei [177].

The development of MQMAS by Frydman and Harwood somewhat diminished the enthusiasm for DAS and DOR, because this pulse sequence enabled high-resolution spectra of quadrupolar nuclei to be obtained under magic angle spinning [178]. This method, with the related STMAS experiment $[179,180]$, has been preferentially adopted by most groups working on highresolution spectroscopy of half-integer quadrupolar nuclei because it can be performed with standard instrumentation. Off the magic angle, COASTER explicitly separates the CSA and quadrupolar anisotropies by performing 
a single to triple quantum correlation while spinning the sample at $70.1^{\circ}$ [181]. More recent DOR implementations generally focus on experimental parameters that cannot be obtained using MQMAS or STMAS. For example, Bryce and co-workers have demonstrated its utility for extracting dipolar couplings and J-couplings from materials in which quadrupolar broadening would obscure such interactions in the absence of DOR [182, 183, 184, 185]. Incompletely averaged homonuclear dipolar couplings change the lineshapes in MQMAS and thus provide additional structural information [186]. Modern implementations of both DAS [187] and DOR have benefitted from techniques such as RAPT $[188,189]$ for enhancing sensitivity for quadrupolar nuclei. In another approach, off-magic angle spinning is used in the context of exchange experiments to reintroduce dipolar interactions or CSA, allowing the relative positions of structural units in a material $[190,191]$. Kentgens and co-workers have improved the resolution of such experiments by combining some aspects of the DAS and MQMAS experiments to produce the exchange experiments $\mathrm{MQP}_{4} \mathrm{MAS}$, in which the sample is spun at $30.6^{\circ}$ or $70.1^{\circ}$ during an MQMAS experiment, directly reintroducing the homonuclear dipolar coupling, which is averaged out in traditional MQMAS. Similarly, the sample is spun at the magic angle at all times except during the added mixing period, which reintroduces homonuclear dipolar interactions in a 2D MQMAS experiment [127]. MQOMAS provides higher resolution, since detection occurs at the magic angle, but $\mathrm{MQP}_{4} \mathrm{MAS}$ is easier to implement and works better in the case of large quadrupolar interactions.

One advantage of solid-state NMR generally and VAS in particular is its ability to separate the effects of multiple spin interactions in complex spectra. This has been productively used in the study of phosphine complexes, which play an important role in organometallic chemistry due to their synthetic versatility and their ability to form complexes with a wide variety of metals in different oxidation states. ${ }^{31} \mathrm{P}$ NMR is frequently used to eludicate their structures. In solution, where chemical shifts are averaged to the isotropic value, nuclei with the same chemical shift do not have observable $J$-couplings. Crystals of metal phosphine complexes often have complex solid-state NMR spectra, because they commonly contain dipole-dipole coupled pairs of ${ }^{31} \mathrm{P}$ nuclei in magnetically inequivalent sites. In the solid state, atomic sites can be crystallographically but not magnetically equivalent. Two sites are crystallographically equivalent when they are related any symmetry operation, but magnetically equivalent only when related by translation or 
inversion operations: all magnetically equivalent spin pairs are also crystallographically equivalent, but the converse is not necessarily true $[192,193]$. In practice this manifests itself as a pair of nuclei that have the same chemical shift tensor, but are oriented differently, leading to spectral splittings arising due to interference between the homonuclear dipolar coupling and CSA [194].

Understanding the detailed molecular structure and bonding patterns in these complexes is important for investigating reaction mechanisms. In such systems, complete interpretation of the solid-state NMR spectra requires measurement of the relevant $J$-couplings and dipolar couplings, the principal components of each CSA tensor, and the Euler angles relating the CSA tensors to the dipolar reference frame. Different approaches have been used to separate the effects of these interactions, including varying the MAS frequency and the applied magnetic field, e.g. in [195]. Symmetry-based sequences can be used to determine the relative orientations of two CSA tensors under slow MAS, as demonstrated for polycrystalline solids [196]. In the fast spinning limit, the higher order terms involving interactions among the CSA and dipolar couplings are averaged out and the separation between the peaks approaches the isotropic chemical shift difference. At lower spinning speeds, where $\nu_{r}<\nu_{C S A}$, the effects of these cross terms are observed and the magnitude of the shifts involved depends on the product of the CSA and the dipolar couplings. The shifts are therefore dependent on both the spinning speed and the applied magnetic field, up to the point where the isotropic chemical shift difference is large enough to suppress this effect.

The approach described above has also been combined with a $2 \mathrm{D} J$ resolved experiment, which was used to resolve indirect ${ }^{31} \mathrm{P} \_{ }^{31} \mathrm{P} J$-couplings in $\mathrm{Cu}\left(\mathrm{PPh}_{3}\right)_{2} \mathrm{NO}_{3}$ and $\mathrm{Cu}\left(\mathrm{PPh}_{3}\right)_{2} \mathrm{BH}_{4}$ [197]. In each of these systems, the two ${ }^{31} \mathrm{P}$ nuclei have the same CSA tensor principal components, but their orientations are different. Furthermore, broadening is induced by the interactions of the ${ }^{31} \mathrm{P}$ nuclei with neighboring ${ }^{63} \mathrm{Cu}$ and ${ }^{65} \mathrm{Cu}$ nuclei; the quadrupolar interactions of the latter are large enough that their quantization axes are incompletely aligned with $\mathrm{B}_{0}$. These factors often make it impossible to resolve the ${ }^{31} \mathrm{P}-{ }^{31} \mathrm{P} J$-couplings from a $1 \mathrm{D}$ experiment, whereas the $2 \mathrm{D}$ $J$-resolved experiment provides a clean measurement of $J$, correlated with the ${ }^{31} \mathrm{P}$ chemical shift.

A comprehensive study of methods that can be used to separate the ef- 
fects of the many interacting Hamiltonians governing the behavior of these systems concluded that orientation-dependent solid-state NMR of a single crystal provides the most complete information from a single data set [198]. However, this experiment is not always practical because it requires a specialized probe with a goniometer head [199], not to mention a large single crystal of the sample. Alternatively, a variety of solid-state NMR techniques using powder samples can be used. Eichele et al. found that a combination of rotational resonance experiments under fast MAS (to measure the dipolar coupling), 2D spin echo experiments (to correlate the $J$-coupling with the chemical shift), and VAS (to observe the scaling of anisotropic interactions) could be used to determine all of the relevant parameters. The use of off-magic-angle spinning enables measurement of the CSA parameters with spinning speeds larger than the magnitude of the CSA. The very thorough study by Eichele et al. provides a comprehensive account of the advantages and disadvantages of each of these techniques, and how they can effectively be used in combination [198].

The study of such systems is difficult; however according to the treatment of Grandinetti, traditional phase cycling can still be used to select coherence transfer pathways when the eigenstates are not aligned with $B_{0}$, provided that the coherences are defined in the diagonal frame of the unperturbed, time-averaged interaction [200]. Under sample spinning, the relative orientations of this tilted rotating frame with the laboratory frame are timedependent [201]. A very thorough description of how to describe the relevant PAS frames, construct the tensor representations and generate the symmetry pathways for many of the experiments described in this review was given by Grandinetti et al. [202].

The idea of using analysis of VAS lineshapes to extract the $J$-couplings, dipolar couplings, and CSA information from a pair of coupled, crystallographically equivalent but magnetically nonequivalent spins was explored further by Wu and Wasylishen [203, 204]. A VAS version of the 2D $J$-resolved experiment was used to determine the $J$-coupling between two ${ }^{31} \mathrm{P}$ nuclei bonded to an $\mathrm{Hg}$ atom and related by a $C_{2}$ symmetry axis. An important advantage of this experiment is that it determines the $J$-coupling independently from the dipolar coupling, simplifying the analysis. Although the full VAS spectrum of a powder samples represents a complicated sum of many $A B, A X$, and $A_{2}$ spectra, the projection in the indirect dimension of the $2 \mathrm{D}$ 
$J$-resolved experiment always gives the pure $J$-coupling.

Although single-crystal NMR is rarely used because of its demanding instrumentation and sample preparation requirements, it is extremely rich in information and offers significant advantages over the use of sideband analysis under slow MAS conditions. Because the dipolar couplings are continuously scalable with the spinning angle, variable angle experiments are applicable to a wide range of samples with different dipolar coupling values. This can be implemented by simple reorientation of a static sample in the case of a single crystal, or with VAS for a polycrystalline or powder sample. Furthermore, if second-order effects are considered, VAS also enables measurement of the relative orientations of the CSA tensors between two coupled spins [204].

\section{Applications: Liquid Crystals}

In solution-state NMR, residual dipolar couplings [205, 206] and CSA $[207,208]$ can be measured by inducing alignment in the molecule of interest via steric interactions with anisotropic media (e.g. filamentous phage or bicelles) that align in a strong magnetic field due to their magnetic susceptibility anisotropy $\Delta \chi$. The solution-state RDC experiment has proven extremely useful for obtaining long-range distance constraints in solution [16], but it is limited to the case of very weak alignment. This is due to both the low power handling capability of solution-state probes and the fixed delays in the pulse sequences, which do not work if the splittings deviate too far from $J$. VAS and SAS enable the investigation of many interesting samples at the interface between solid-state and solution NMR. Here the strength of the dipolar couplings is modified by changing the spinning axis of a strongly aligned sample rather than comparing experiments performed in weakly aligned media and isotropic liquids. This allows exquisite control over the dipolar interactions, because they can be scaled anywhere between their full value at $0{ }^{\circ}$ and zero at the magic angle. The scaling of the anisotropic interactions induced by VAS or SAS depends only on the spinning angle [209], and avoids artifacts due to strong interaction with the ordering medium that can complicate interpretation of data where the orientation is scaled by dissolving a solute molecule in different thermotropic $[210,211,212]$, but not lyotropic $[213,214]$ liquid crystals. 
(a)

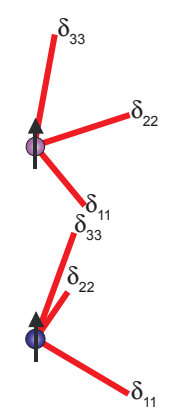

CSA PAF (b)

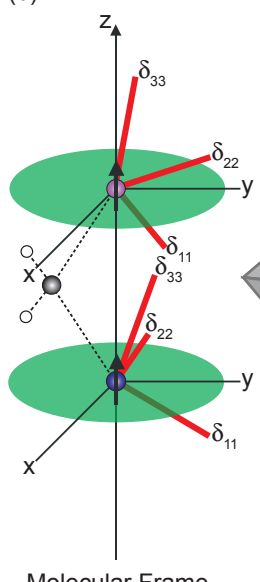

(c)

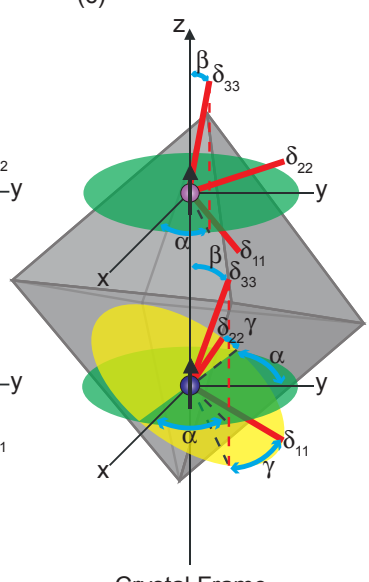

(d)

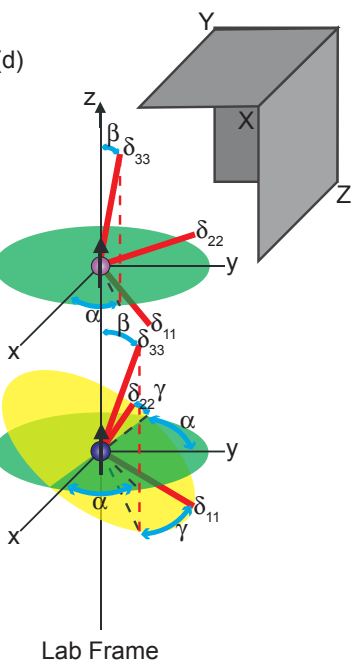

Figure 14: (a) Two $\mathrm{P}$ nucleii showing their respective CSA tensors (b) the two $\mathrm{P}$ nuclei along the internuclear vector (c) the molecules align in a crystal phase (d) the lab frame shares the $\mathrm{X}$-axis with the crystal frame and is inverted along the $\mathrm{Y}$ - and $\mathrm{Z}$-axes.

The seminal observation by Saupe that the liquid crystal director aligns in a magnetic field, and that anisotropic interactions can be scaled by reorienting the director [215], has led to a range of applications in chemistry, materials science, and molecular biology. Upon spinning a magnetically oriented liquid crystal sample at a speed greater than the critical speed $\omega_{c}$, the alignment is dominated by the torque produced by spinning. When the rotor frequency $\omega_{r}$ is greater than the critical speed $\omega_{c}$, the director rotates about the rotor axis with frequency $\omega=\sqrt{\left(\omega_{r}^{2}-\omega_{c}^{2}\right)}$. The critical speed for a thermotropic liquid crystal is given by

$$
\omega_{c}=\left|\frac{\Delta \chi B_{0}^{2}}{2 \mu_{0}^{3} \gamma_{1}}\right|
$$

where $\gamma_{1}$ is the twist viscosity coefficient, describing the coupling between the rotation of the director and the fluid, which depends on physical parameters such as the length of the polymer chain, the degree of coupling between the main chain and side chains, and both the absolute and reduced temperatures of the system. The reduced temperature $T / T_{c}$ of a nematic liquid crystal describes how close the system is to its nematic-to-isotropic transition temperature, and hence relates the order parameter of the system in a way 
that is comparable across different liquid crystals [216]. The critical speed for typical liquid crystals is much lower than the MAS rates routinely used in solid-state NMR with standard instrumentation. This framework is helpful for thinking about the connections between the temperature-dependent and spinning-dependent changes in liquid crystal orientation. In general, a liquid crystalline preparation that aligns over a wide range of temperatures is also robust with respect to sample spinning.

The molecules align parallel or perpendicular to the spinning axis depending on the sign of $\Delta \chi$ [217], and changing the spinning axis reorients the liquid crystal director, scaling the dipolar couplings [155, 156]. For samples with positive values of $\Delta \chi$, the director orientation is along the rotor axis for spinning angles $0^{\circ}<\beta_{R}<\beta_{m}$, while at angles larger than this with respect to $B_{0}$, the directors fan out in a two-dimensional plane perpendicular to the rotor axis, yielding a powder spectrum. For liquid crystals with negative $\Delta \chi$ values, the opposite dependence is observed, with uniform alignment at angles $90^{\circ}>\beta_{R}>\beta_{m}[151,155]$. The driving force for the alignment is that the average magnetic energy per unit volume $E_{\text {mag }}(\beta, \delta)$ over a rotor period is minimized.

$$
E_{\text {mag }}(\beta, \delta)=-\frac{\Delta \chi}{3 \mu_{0}} B_{0}^{2}\left(\frac{3 \cos ^{2} \delta-1}{2}\right)\left(\frac{3 \cos ^{2} \beta-1}{2}\right)
$$

where $\delta$ is the angle between the liquid crystal normal and the rotor axis, and $\beta$ is the angle between the rotor axis and $B_{0}$.

In practice, this alignment is imperfect, and spinning sidebands are usually observed corresponding to a cone of director distributions distributed around the rotor axis; its spread depends on properties of the liquid crystal, the temperature, and the spinning speed. At the magic angle, the directors are randomly oriented because there is no orienting force; therefore prolonged periods of magic angle spinning eliminate the sample orientation. Near $0^{\circ}$, the spectra of liquid crystalline samples are often second-order, meaning that the dipolar couplings are of comparable magnitude to the relevant chemical shift differences. Obtaining useful structural information from strongly oriented samples thus requires finding the balance between having strong enough alignment to observe the relatively weak couplings between distant spins and ensuring that the spectrum is simple enough for unambiguous spectral assignment. VAS and SAS experiments make this possible by examining 
the same sample with different orientational scaling of the anisotropic interactions, either in a series of $1 \mathrm{D}$ spectra or in a multidimensional isotropicanisotropic correlation.

The dependence of $\mathrm{CH}$ dipolar couplings (D) on the spinning angle is plotted for different degrees of alignment in Fig. 15. The net splitting in the figure accounts for the sign of $\Delta \chi$, the FSLG scaling factor, and the order parameter $S_{z z}$, and is given by $\Delta_{C H}=0.571\left(J-\Delta \chi \cdot S_{z z} \cdot P_{2}(\cos \beta) \cdot D\right)$. $\mathrm{D}$ values are negative for $\mathrm{CH}$ spin pairs, and $\Delta \chi$ is negative for unmodified DMPC/DHPC bicelles. Many thermotropic liquid crystals (e.g. I52) have $\Delta \chi>0$, and $\Delta \chi$ can be made positive for bicelles by doping them with lanthanides. For $\Delta \chi<0$ bicelles, spinning at $\beta>\beta_{m}$ can be advantageous. Here $P_{2}(\cos \beta)<0$, which cancels the negative sign of D and causes J and the scaled dipolar couplings to add instead of subtract, which is convenient for interpretation. Also, the couplings are scaled by a factor of two such that the range of net splitting values is scaled to fit within $\sim 2000 \mathrm{~Hz}$. For separated local field experiments like SAS HETCOR [121], where the ${ }^{1} \mathrm{H}$ chemical shift information is preserved along with the $\mathrm{CH}$ couplings, allowing the magnitude of the splittings to be too large will require opening up the spectral width in the indirect dimension. This may reduce resolution to the point where the smaller couplings, corresponding to interesting distances, are no longer measurable.

Non-spinning variable angle-NMR (VA-NMR) provides the same type of information for solutes incorporated in stretched polymer gels. Scaled CSAs and quadrupolar couplings have been measured using static samples in commercial VAS and MAS probes using manual setting of the angle [219]. This method shares some of the advantages of VAS with an appropriately chosen coil: the technique can explore the full range of angles between $0^{\circ}$ and $90^{\circ}$ (and therefore scaling factors on the residual, partially motionally averaged CSA ranging from 1 to $-1 / 2$ ), and all experiments are performed on a single sample without introducing a phase transition. Other methods exist for reintroducing anisotropic interactions to a sample dissolved in a polymer gel, such as stretching the gel in a silicone tube [220] or compressing it using the plunger of a Shigemi tube [221]. These methods effectively reintroduce RDCs, but may be less than ideal for RCSA measurements, as squeezing the gel, with its concomitant removal of solvent, can generate large, sometimes unpredictable effects on the chemical shift. Some practical aspects of VA- 


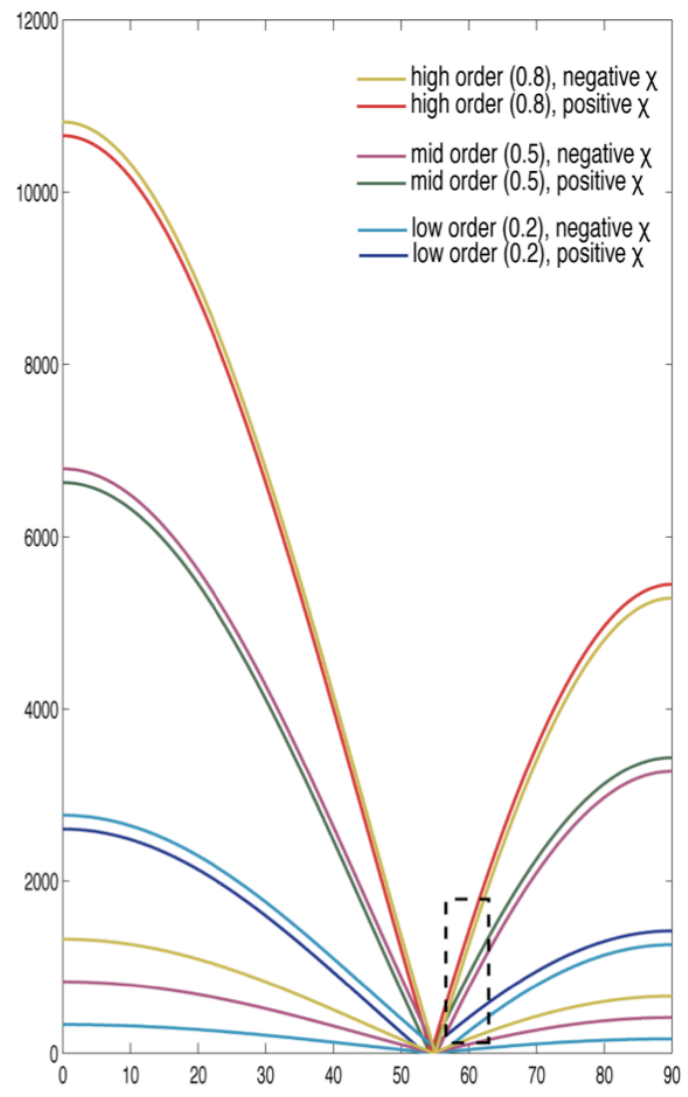

Figure 15: Net CH splitting expected in SAS HETCOR spectra for oriented samples as a function of rotor angle $\beta$. The top 6 curves (above $2000 \mathrm{~Hz}$ splitting) are for directly bonded $\mathrm{CH}$ pairs: dipolar coupling constant (rigid solid) $\mathrm{D}=-23,500 \mathrm{~Hz}$. The lower 6 curves (below $2000 \mathrm{~Hz}$ splitting) are for nonbonded $\mathrm{CH}$ pairs with the next strongest $\mathrm{CH}$ coupling: $\mathrm{D}=-2900 \mathrm{~Hz}$. The dashed black box shows a range of spinning angles $(\beta>$ $\beta_{m}$ ) for measuring attenuated dipolar couplings while measuring $(\mathrm{J}+\mathrm{D})$ rather than (J-D). Figure reprinted from [218].

NMR are more straightforward than VAS due to the lack of sample spinning. However, obtaining accurate residual CSA measurements does require careful attention to the preparation of polymer sample and the chemical shift referencing.

\subsection{Thermotropics}

VAS experiments have been used in the study of thermotropic liquid crystals, both per se and as partially ordering NMR solvents, since the 1980s [151, 222]. NMR on liquid crystals is a powerful technique that can be exploited to determine many particulars of molecular structure, due to the sensitivity of the anisotropic interactions (e.g. dipolar couplings, CSA, and quadrupolar interactions in nuclei with $I>1 / 2)$ to the local chemical environment, as reviewed in [155]. Spinning within a few degrees of the magic angle scales the dipolar couplings such that they are comparable to the $J$-couplings and simplifies the fiendishly complicated second-order spectra 
obtained at $0^{\circ}[150]$. Careful measurements of the ${ }^{1} \mathrm{H}-{ }^{1} \mathrm{H}$ and ${ }^{1} \mathrm{H}-{ }^{13} \mathrm{C}$ scalar and dipolar couplings can be used to get the five order parameters required to derive the complete molecular ordering tensor [223].

When chiral liquid crystals are used, determining the ordering tensor also provides information about the stereochemistry and allows unambiguous assignment of protons or deuterons in chiral or proctorial molecules [224]. In chiral liquid crystals, interactions of the solute with the low-symmetry mesogen can result in effective lowering of the symmetry of even rigid solute molecules [225]. Thermotropic liquid crystals provide relatively strong alignment, breaking the degeneracy in the NMR spectra of small, simple molecules, as exemplified for methyl iodide in ZLI 1167 [157]. This enables highly precise measurements of NMR parameters relating to the details of molecular structure, including $\mathrm{D}_{H H},{ }^{1} J_{C H}$, the $\mathrm{HCH}$ bond angle, and the proton CSA. By comparing to experiments performed using isotropic samples, the sign and magnitude of the difference between $J_{C H}$ and $D_{C H}$ can be determined.

These VAS experiments can therefore be considered precursors to the solution-state RDC experiments commonly used in the determination of macromolecular structure [226], and a natural extension of early orientedsample experiments to determine the anisotropy of ${ }^{13} \mathrm{C}-{ }^{13} \mathrm{C}$ and ${ }^{13} \mathrm{C}-{ }^{15} \mathrm{~N}$ $J$-couplings [227] and the ${ }^{1} \mathrm{H}$ and ${ }^{13} \mathrm{C}$ CSA values [228] for model compounds dissolved in liquid crystals. Producing alignment of solutes by modifying the phase of the solvent, whether by changing the temperature or the concentrations of the components, is subject to the limitation that the RDCs, RQCs, and scaled CSA values can only be scaled over a finite range before the liquid crystal changes phase. In contrast, VAS experiments enable the anisotropic interactions to be scaled over the full range from their unscaled values to 0 at the magic angle. This methodology has been demonstrated in applications ranging from small organic molecules [229, 230] and model compounds [121] to peptides associated with a membrane mimetic [92].

Separated local field (SLF) experiments have been applied to determining segmental order parameters in nematic [231] and lyotropic liquid crystals $[22,232,23]$. The combination of separated local field (SLF) and VAS experiments has been used to characterize the order parameters of a variety of nematic and smectic liquid crystals, including 4-n-alkyl-4'-cyanobiphenyls 
[233, 234, 235] and 4-n-alkoxy-4'-cyanobiphenyls [236, 235], bicyclohexylnitriles [237], and phenylcyclohexanes [234]. This methodology has also been used to explore the effects of solute molecules on the ordering of 4-n-alkyl4'-cyanobiphenyls [238]. Although the relevant data could be obtained from VAS experiments alone, here they are supplemented with SLF data in order to avoid systematic errors in the measurement of the chemical shift induced by errors in setting the angle. Even in the case where the value of the CSA is small, angle mis-sets can affect the value of the isotropic chemical shift $\delta_{i s o}$ because it is obtained from the observed chemical shift $\delta_{o b s}$, which is determined by both the order parameter $\mathrm{S}$ and the anisotropic chemical shift.

$$
\begin{gathered}
\delta_{o b s}=\delta_{i s o}+\delta_{\text {aniso }} \\
\delta_{\text {obs }}=\delta_{\text {iso }}+\frac{1}{3} S_{z z}\left[\sigma_{z z}-\frac{1}{2}\left(\sigma_{z z}+\sigma_{y y}\right)\right]+\frac{1}{3}\left(S_{x x}-S_{y y}\right)\left(\sigma_{x x}+\sigma_{y y}\right) \\
+\frac{2}{3} S_{x y} \sigma_{x y}+\frac{2}{3} S_{x z} \sigma_{x z}+\frac{2}{3} S_{y z} \sigma_{y z}
\end{gathered}
$$

For molecules with a minimum degree of symmetry, this can be considerably simplified because the off-diagonal terms of $S$ are zero, and $S_{x x}-S_{y y}$ is negligible and temperature-independent [239].

The chemical shift for a molecule in a liquid crystalline phase aligned parallel with the magnetic field can be calculated from $\delta_{o b s}$ at angle $\theta$ using the relation

$$
\begin{gathered}
\delta_{L C}-\delta_{i s o}=\frac{2\left(\delta_{o b s}-\delta_{i s o}\right)}{\left(3 \cos ^{2} \theta-1\right)} \\
\delta_{o b s} \approx \delta_{i s o}+S_{z z} \frac{2}{3}\left(\sigma_{z z}-\frac{\sigma_{x x}+\sigma_{y y}}{2}\right)+b
\end{gathered}
$$

where $b$ is an empirical constant.

Unless the molecules under investigation have high symmetry, two or more order parameters are required to define the orientation of the liquid crystal, and in general the axis system of the chemical shift tensors has an unknown relationship to that defining the order parameters. The chemical shift of an oriented liquid crystal molecule is related to the order parameters by

$$
\delta_{L C}=\delta_{i s o}+\Sigma_{\alpha, \beta} S_{\alpha \beta} \sigma_{\alpha \beta}
$$


where $\delta_{i s o}$ is the isotropic chemical shift, $S_{\alpha \beta}$ is the order parameter along the $\alpha \beta$ axis, and the elements $\sigma_{\alpha \beta}$ are the elements of the CSA tensor in the coordinate system of the order parameters [240]. In principle, it should be possible to straightforwardly obtain the order parameters from the CSA values. However, as pointed out by an early study, without an unambiguous determination of the CSA tensor from single crystal studies or a very large number of chemical shift measurements, in practice the results are usually only approximate because $\delta_{L C}$ does not have a strong dependence on all components of the ordering matrix, resulting in large uncertainties [241]. The combination of VAS and SLF experiments, performed at different temperatures in order to change the molecular ordering, shows a linear dependence of the order parameter of each rigid molecular segment on the chemical shifts of the associated carbons [235]. The experimental factors thusly obtained provide a semi-empirical framework for calculating order parameters for mesogens of the same type (in this case 4-n-alkyl-4'-cyanobiphenyls and 4-n-alkoxy-4'-cyanobiphenyls ), given their ${ }^{13} \mathrm{C}$ chemical shifts. This methodology was then used to tabulate order parameters for several different types of mesogens forming nematic and smectic phases [239], including low-viscosity fluorinated molecules [242] and those having different shapes from the more commonly used rod-like molecules [243]. The values measured for several relatively symmetric liquid crystalline molecules made it possible for researchers who lack the specialized equipment necessary for VAS to determine the order parameters in related liquid crystalline preparations using only the ${ }^{13} \mathrm{C}$ chemical shifts.

The spectra of even relatively simple molecules become very complicated when they are dissolved in chiral liquid crystalline phases, because the effective lowering of molecular symmetry introduces couplings that would otherwise not be observed. Many techniques exist for simplifying the analysis of such spectra by selecting particular types of couplings. Bilinear rotational decoupling (BIRD) modules [244] can be inserted into the middle of the $t_{1}$ evolution period of a heteronuclear correlation experiment to select large, one-bond heteronuclear couplings [245]. Homonuclear and heteronuclear selective refocusing experiments (SERF and HetSERF) can be used to distinguish between R- and S- enantiomers on the basis of the different chemical environments (and hence different dipolar couplings) experienced by these molecules in a chiral liquid crystalline phase [246, 247]. These experiments are modified 2D J-resolved sequences that use selective pulses to select the 
spectrum of one enantiomer or the other, based on differences in their ${ }^{1} \mathrm{H}-{ }^{1} \mathrm{H}$ or ${ }^{1} \mathrm{H}-{ }^{13} \mathrm{C}$ dipolar couplings. In this application, VAS is used near the magic angle to ensure that the ${ }^{1} \mathrm{H}$ spectrum is sufficiently resolved for the selective pulses to work [156].

Remarkably, it is possible to change the director axis of a liquid crystal solution through a range of $90^{\circ}$ by mixing two nematic phases with opposite values of $\Delta \chi$ and varying the relative amounts of each component [248]. Powder patterns consistent with the scaled CSA value are observed, until the critical concentration, where a jump in chemical shift indicates a $90^{\circ}$ shift in the liquid crystal director orientation [249]. In such mixtures, the spinning speed and the rotor axis also influence the director dynamics, indicating that careful attention to experimental parameters is required [250].

VAS experiments are problematic in cholesteric phases, in which the director is helical. Although these phases can be used to orient solutes, upon exposure to a magnetic field, it is the director helices that orient parallel or perpendicular to $B_{0}$, leading to a powder pattern in the solute spectrum that requires multipulse decoupling methods to simplify [251]. An interesting and useful exception is presented by solutions of poly-( $\gamma$ benzyl-L-glutamate) (PBLG). PBLG forms a cholesteric phase in the absence of a magnetic field, but in this case an external field causes the helices to untwist, forming a chiral nematic phase. This can lead to very interesting hydrodynamic behavior, such as the observation, using ${ }^{2} \mathrm{H}$ VAS experiments, of a second, higher critical speed in this phase, possibly corresponding to supramolecular helical ordering being re-established at higher rotor speeds. Above the upper critical speed ( $350 \mathrm{~Hz}$ under these experimental conditions), the spectra are again consistent with the directors being distributed over a range of orientations, but independent of the rotor angle [252]. This effect highlights an important instrumental challenge of working with such liquid crystalline samples - the critical speeds are often on the order of tens to hundreds of $\mathrm{Hz}$, which is much slower than the MAS or VAS rates used for typical samples.

SAS experiments on small molecules dissolved in strongly oriented thermotropic liquid crystals have been used to produce isotropic-anisotropic correlations in which the isotropic chemical shift is correlated to a first-order spectrum obtained by spinning slightly off the magic angle. SAS and VAS experiments on oriented liquid crystals have been used to obtain CSA values 
correlated with isotropic chemical shifts [253]. The SAS-COSY experiment is a modified COSY-45 sequence where the angle switch from $25^{\circ}$ to the magic angle occurs between the two $45^{\circ}$ pulses [254]. The off-magic angle value was chosen to give easily measurable splittings for the desired couplings, while minimizing spin diffusion as much as possible by staying relatively near the magic angle. For best performance of this experiment, it is necessary to minimize the hopping time, during which relaxation and spin diffusion reduce the signal, and to perform the direct detection slightly away from the magic angle $\left(0.1^{\circ}\right.$ is sufficient), because prolonged spinning at the magic angle destroys the sample orientation. A more involved variation of this experiment requires two angle switches; here, the isotropic spectrum is correlated with the sidebands obtained by spinning the sample perpendicular to the magnetic field, where the liquid crystal directors are spread out in a plane perpendicular to the spinning axis [255].

\subsection{Lyotropics}

"Bicelle" mixtures are frequently used in both solution and solid-state NMR as alignment media and membrane mimetics. These versatile mixtures produce a variety of self-assembled structures with useful properties, and are comprised of varying proportions of a long-chain lipid and a short-chain lipid. The molar ratio of long-chain to short-chain is called the $q$ factor. The most commonly used and best-characterized preparation is a mixture of dimyristoyl phosphatidylcholine (DMPC) and dihexanoyl phosphatidylcholine (DHPC) [256]. Like all lyotropic liquid crystals, these mixtures have very complicated phase diagrams with respect to temperature and composition. In general, more short-chain lipids result in a solution of isotropic micelles, while more long chain lipids correspond to an increase in degree of alignment (anisotropic bicelles, lipid bilayers) and a decrease in alignment temperature [257]. Anisotropic bicelles align in a magnetic field, acting as a weak alignment medium to reintroduce scaled anisotropies in solution state NMR [258, 259, 260, 207]. Other bicellar systems have modifications including increased acyl chain lengths to accommodate larger hydrophobic regions in certain transmembrane proteins [261] and addition of different head groups to make negatively or positively charged bicelles $[262,263]$. Further studies have showed improved stability of model membranes by using a variety of lipid components [264, 265]. In the context of NMR experiments bicelles have usually been visualized as either discoids similar to the unoriented bi- 


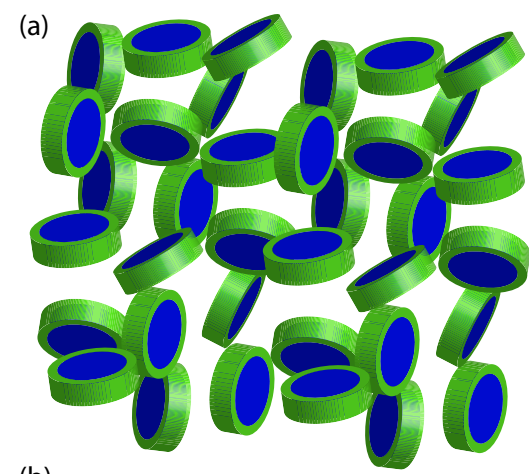

(b)

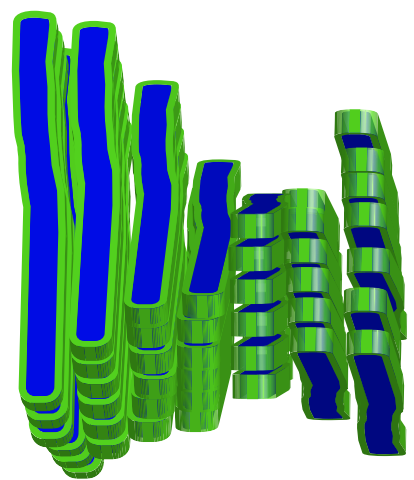

Figure 16: (a) Unoriented bicelle discs of the type used to solubilize membrane proteins for solution-state NMR. (b) Wormlike micelles making up a chiral nematic phase. Based on neutron scattering data [266], this is thought to be the predominant phase of the DMPC/DHPC mixtures most frequently used in alignedsample NMR.

celles often used for solubilizing membrane proteins, or perforated lamellae; in fact, several oriented phases exist, and evidence from small angle neutron scattering and optical microscopy indicates that one of the most often used aligned phases is actually a chiral nematic phase [266]. A schematic of unoriented bicelles and wormlike micelles, both of which can be produced by DMPC/DHPC mixtures at different concentrations, is shown in Figure 16. Order parameters of membranes can be obtained using ${ }^{2} \mathrm{H}$ spectra of selectively labeled samples, by using dipolar recoupling sequences such as DROSS [267] or specialized symmetry-based sequences [22, 232, 268].

In the absence of sample spinning, the standard DMPC/DHPC bicelle mixture orients with its director perpendicular to $B_{0}$, which is not necessarily the most convenient orientation. The addition of lanthanide ions with a positive $\Delta \chi$ value flips the orientation of the bicelle phase by $90^{\circ}[269,270,271]$, but this can cause other problems, such as paramagnetic shifts and/or broadening of some peaks. VAS is a less intrusive method for aligning the bicelles, as they align upon spinning in the same manner as other liquid crystalline 


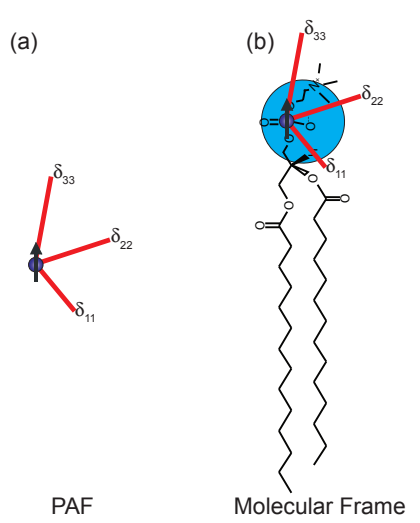

(d)

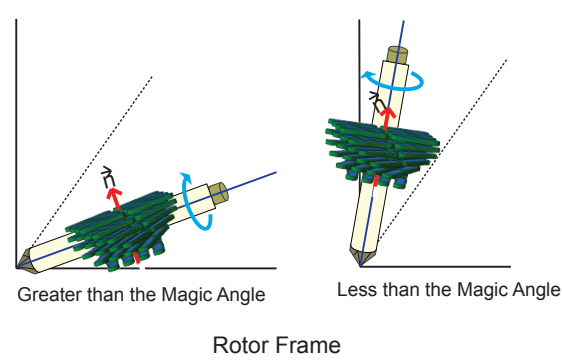

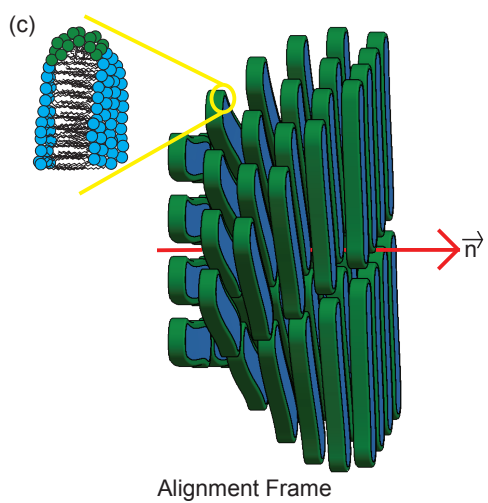

(e)

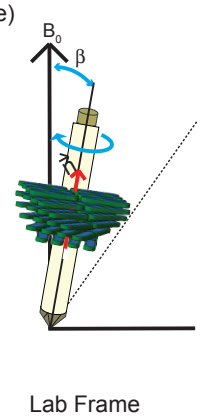

Figure 17: (a) A phosphorus nucleus showing its CSA tensor, (b) the ${ }^{31} \mathrm{P}$ nucleus and its CSA in position in the head-group of the DMPC, (c) alignment of wormlike micelles showing director. (d) Wormlike micelles align with the director perpendicular to the rotor axis when the spinning angle is greater than the magic angle and parallel to the rotor axis when the spinning angle is less than the magic angle. (e) The wormlike micelles in the rotor are oriented with respect to the external magnetic field.

samples $[159,272]$, giving dipolar coupling values intermediate between those of solids and weakly oriented liquids. This methodology is therefore applicable to both the "solution-type" RDC experiments where soluble molecules are aligned via weak interactions with the bicelles [273], and in "solid-state" type experiments where the bicelle mixtures provide a membrane mimetic that enables membrane proteins to insert [274].

Differential line broadening (DLB) of the ${ }^{13} \mathrm{C}$ resonances of methyl groups in the absence of ${ }^{1} \mathrm{H}$ decoupling has been proposed as a reporter of bicelle alignment [275]. This effect arises from interference between ${ }^{13} \mathrm{C}$ CSA and ${ }^{1} \mathrm{H}-{ }^{13} \mathrm{C}$ dipolar couplings, and is the basis of the well-known methyl TROSY effect [276]. Although analysis of DLB in methyl groups of proteins in strongly oriented bicelle samples may provide insight into the organization of the liquid crystal domains, its use in this context has not yet been fully explored [277]. An alternative easy, inexpensive method for assessing bicelle 
orientation is to introduce a relatively small amount of $\mathrm{D}_{2} \mathrm{O}$, which becomes partially oriented due to time-averaged interaction with the liquid crystal phase [278]. This produces a residual quadrupolar coupling that gives rise to a doublet in the ${ }^{2} \mathrm{H}$ spectrum, with spacing

$$
\Delta \nu=-\frac{3}{8 \pi} Q_{33} S_{33}
$$

for a static sample, where $Q_{33}$ is the component of the quadupolar interaction along the director, and $\mathrm{S}_{33}$ is the order parameter. For a spinning sample, this is scaled by factors of $P_{2}(\cos \beta)$ for both the director-rotor axis angle and the rotor axis- $B_{0}$ angle:

$$
\Delta \nu=\frac{3}{4 \pi} Q_{33} S_{33} P_{2}(\cos \delta) P_{2}(\cos \beta)
$$

In the first application of VAS and SAS methodology to bicelle samples, Zandomeneghi et al. verified that bicelle mixtures behave as expected for a lyotropic liquid crystal with negative $\Delta \chi$ under sample spinning, with $\delta=90^{\circ}$ for $0 \leq \beta<\beta_{m}$, and $\delta=0^{\circ}$ for $\beta_{m}<\beta \leq 90$ [159]. Consistent with previous investigations of other liquid crystals [150, 279, 252], the bicelles were found to have two critical speeds for the rotor frequency $\nu_{r}$. When spinning between $90^{\circ}$ and the magic angle at a speed above the first critical frequency but below the second one, the director was oriented parallel to the rotor axis. When the rotor frequency exceeds the second critical frequency, an additional torque that scales as the square of the rotor frequency comes into play, which appears to stabilize the $\delta=0^{\circ}$ orientation. In the system studied, this second torque was found to be the dominant factor at rotor angle $\beta$ where $P_{2}(\cos \beta)$ was scaled almost to 0 . This study also established that SAS can be used to investigate liquid crystalline samples in metastable orientations, e.g. bicelles with the director parallel to $B_{0}$ in the absence of lanthanide dopants. The relaxation time of the metastable phase back to the equilibrium state was found to be about $0.6 \mathrm{~s}$, much longer than the switching time of $50 \mathrm{~ms}$ achievable with a servo motor. Furthermore, the introduction of a membrane-bound peptide (here, a transmembrane helix domain of an acetylcholine receptor) did not interfere with the bicelle alignment. This type of SAS methodology was also used to measure RDCs in a model system consisting of the Lenk peptide embedded in DMPC/DHPC bicelles, establishing the feasibility of this type of study for membrane proteins in a native-like 
environment [280].

The phase behavior and dynamics of the bicelle system depend on the spinning dynamics in complex ways [277]. Analysis of the ${ }^{31} \mathrm{P}$ CSA provides information about the degree of orientation and the mosaic spread $\mu$. The bicelle sample is assumed to consist of several large domains, each with its own director orientation with respect to the rotor frame. The distribution of these orientations is characterized by a Gaussian function of the angle $\beta$ centered at its average value $\bar{\beta}$ with standard deviation $\mu$.

$$
p(\beta)=\frac{1}{\mu \sqrt{2 \pi}} \exp \left[-\frac{1}{2}\left(\frac{\beta-\bar{\beta}}{\mu}\right)^{2}\right]
$$

If the directors of all the domains point in the same direction, $\mu=0$.

Looking at some limiting cases, a perfectly ordered sample with $\bar{\beta}=90^{\circ}$ has only even-order sidebands, while one at $\bar{\beta}=0^{\circ}$ has no sidebands. Samples with non-vanishing mosaic spread have all the sidebands of even and odd order, and the sideband patterns display sensitive dependence on $\mu$. For samples spinning away from the magic angle, the mosaic spread of the particular DMPC/DHPC mixture used was found to be about $7^{\circ}$, which is comparable to values measured for DMPC bilayers mechanically oriented on glass plates, both for DMPC alone [281] and in the presence of a membrane-inserting peptide [282]. Higher spinning frequencies increase the mosaic spread, as does approaching the magic angle. The latter effect is an important experimental consideration, because the tendency of the bicelle mixtures to form a powder pattern when close to the magic angle results in a non-linear dependence on $P_{2}(\cos \beta)$. This experimental limitation can be overcome by using SAS experiments that only approach the magic angle for a short time, as in [280], or by using the projected magic angle spinning (p-MAS) technique [283, 108]. In p-MAS, a 2D SAS experiment is performed where neither of the two spinning angles is the magic angle, and the isotropic spectrum is reconstructed along a projection that depends on the particular axes chosen. This enables the isotropic chemical shift to be obtained, along with anisotropic spectra at two different angles, all without approaching the magic angle and thus compromising the sample orientation. The drawbacks of this approach relate to the reconstruction of the isotropic spectrum; because the isotropic spectrum appears along a projection, it is subject to both truncation due to 
the relatively small number of points collected in the indirect dimension, and a scaling factor that depends on the angles. Assuming that the peaks are relatively well-resolved, this need not be a major disadvantage, but for uniformly labeled protein samples, an additional dimension and/or non-uniform sampling approaches may be necessary.

The mosaic spread of bicelle phases is also dependent on the exact composition of the lipid phase as well as the temperature, so characterizing this parameter for the particular membrane or membrane protein system under consideration is necessary. In addition to its obvious utility for studies of membrane-associated peptides and proteins, many of which cannot be easily reconstituted in systems that are suitable for solution-state NMR, or which do not adopt their biologically relevant conformations under such conditions, VAS or SAS experiments can also be used in more traditional RDC measurements on soluble proteins. Here, the bicelle mixtures used are more concentrated in total lipid (e.g. $25 \%-30 \%$ as compared to about $5 \%$ for solution-state experiments). This enables measurement of weaker dipolar couplings arising from long-range interactions, simply by moving the sample to a spinning angle where they are strong enough to be observed. The alignment tensor does not change when the sample is reoriented; the dipolar couplings and/or CSA values are simply scaled according to $P_{2}(\cos \beta)$. Advantageously, when using the spinning axis to scale the RDC values in a strongly aligned sample, the spinning angle can be chosen to measure isotropic $J$ values or dipolar couplings of different magnitudes using a single sample, as was demonstrated for ubiquitin [284]. This procedure has also been used to measure ${ }^{15} \mathrm{~N}$ CSA values over a range of magnitudes for ubiquitin in a strongly aligned bicelle sample [275].

In this and other practical applications of VAS to liquid crystalline samples, care must be taken to measure the spinning angle accurately, because errors in this measurement introduce errors in any experimental parameter that depends on $P_{2}(\cos \beta)$. Another important experimental factor is the difficulty of polarization transfer in strongly oriented, semi-solid samples, which combine some of the inconvenient features of solids and liquids. The relatively strong dipolar couplings in oriented samples render INEPT [285] transfers inefficient, but because the dipolar couplings are partially averaged by random molecular motion (primarily rotational diffusion), cross-polarization efficiency is also poor. Aside from the scaling issues, both INEPT and CP 
suffer in powder samples because their efficiency oscillates with a frequency dependent on the total coupling, such that no single optimum value can be found for a sample with a wide range of couplings. Some different approaches for circumventing this problem include adiabatic-passage CP [92] and the REP-CP approach [286]. Shimming also becomes a problem for SAS experiments because the shims that produce an optimally narrow lineshape at one spinning angle will necessarily be different from those optimized for the other spinning angle, due to the cylindrical shape of the rotor and stator assembly. In VAS experiments the magnetic field can be shimmed at each spinning axis, which is tedious but effective. For SAS, the situation is more complicated, since the angle hopping must be done quickly in the context of a multidimensional experiment. The p-MAS experiment obviates this problem, since the inhomogeneities observed at the different angles are correlated and thus are not observed in the projected spectrum. Various other solutions to this problem have been proposed, such as collecting a $B_{0}$ field map prior to the experiment and then reconstructing the isotropic lineshape using software approaches [287] and switching between two pre-determined shim settings [153]. Using the ultrafast methodology introduced by Frydman et. al, it is possible to collect coupled ${ }^{1} \mathrm{H}_{-}{ }^{13} \mathrm{C}$ or ${ }^{1} \mathrm{H}-{ }^{15} \mathrm{~N}$ HSQC data sets in one scan [288, 289]. This greatly speeds up the process of measuring RDCs for samples of sufficient concentration to provide enough signal for this experiment, because only two spectra need be collected, in the isotropic and anisotropic phases [290]. This could potentially be used in conjunction with VAS, in a series of experiments where single-scan 2D HSQCs are collected over two or more spinning angles, obviating the need for an isotropic sample and allowing this type of experiment to be performed on peptides or proteins that are strongly associated with a membrane.

\section{Outlook}

Motional reorientation experiments have proven useful for a variety of chemical systems. The implementation of these experiments spans a range of difficulty levels from VAS, which can be performed with minor modification of standard MAS instrumentation, to DOR, which requires technically demanding purpose-built probes. As a class of isotropic/anisotropic correlations, the motional reorientation experiments provide great flexibility in separating the effects of different terms in the NMR Hamiltonian, yielding valuable chemical information. With recent developments in NMR, including 
higher field magnets, faster spinning with its concomitant improvements in low-power decoupling and low-power $\mathrm{CP}$, and extensive sample deuteration, these experiments are worthy of further exploration.

\section{Acknowledgments}

R. W. M. acknowledges NSF Grant CHE-1308231 for support of her group's work on the development of VAS and SAS NMR methodology.

\section{References}

[1] P. J. Grandinetti, J. H. Baltisberger, A. . Llor, Y. K. . Lee, U. Werner, M. A. Eastman, A. Pines, Pure-absorption-mode lineshapes and sensitivities in 2-dimensional dynamic angle spinning NMR, Journal of Magnetic Resonance Series A 103 (1) (1993) 72-81.

[2] S. L. Gann, J. H. Baltisberger, A. Pines, Dynamic-angle spinning without sidebands, Chemical Physics Letters 210 (4-6) (1993) 405-410.

[3] J. Klinowski, Spin-off for solid-state studies, Nature 346 (1990) 509 510.

[4] P. Grandinetti, The Encyclopedia of NMR, Wiley, 1996, Ch. Dynamic Angle Spinnng, pp. 1768-1776.

[5] D. B. Zax, dynamic angle spinning and double rotation quadrupolar NMR, Trends in Analytical Chemistry 13 (8) (1994) 300-312.

[6] J. P. Amoureux, NMR characterization of half-integer quadrupolar nuclei in solids, Zeitschrift Fur Naturforschung Section A - A Journal of Physical Sciences 47 (1992) 665-674.

[7] A. P. M. Kentgens, A practical guide to solid-state NMR of half-integer quadrupolar nuclei with some applications to disordered systems, Geoderma 80 (1997) 271-306.

[8] A. Jerschow, From nuclear structure to the quadrupolar NMR interaction and high-resolution spectroscopy, Progress in Nuclear Magnetic Resonance Spectroscopy 46 (2005) 63-78. 
[9] S. E. Ashbrook, M. J. Duer, Structural information from quadrupolar nuclei in solid state NMR, Concepts in Magnetic Resonance Part A 28A (3) (2006) 183-248.

[10] J. P. Amoureux, J. Trébosc, L. Delevoye, O. Lafon, B. Hu, Q. Wang, Correlation NMR spectroscopy involving quadrupolar nuclei, Solid State Nuclear Magnetic Resonance 35 (2009) 12-18.

[11] C. Fernandez, M. Pruski, Probing quadrupolar nuclei by solid-state NMR spectroscopy: Recent advances, Topics in Current Chemistry 306 (2012) 119-188.

[12] B. Deloche, E. T. Samulski, Short-range nematic-like orientational order in strained elastomers: A deuterium magnetic resonance study, Macromolecules 14 (3) (1981) 575.

[13] H. Sass, G. Musco, S. J. Stahl, P. Wingfield, S. Grzesiek, Solution NMR of proteins within polyacrilamide gels: Diffusional poperties and residual alignment by mechanical stress or embedding of oriented purple membranes, Journal of Biomolecular NMR 18 (2000) 303-309.

[14] R. Tycko, F. J. Blanco, Y. Ishii, Alignment of biopolymers in strained gels: A new way to create detectable dipole-dipole couplings in highresolution solid-state NMR, Journal of the American Chemical Society 122 (2000) 9340-9341.

[15] Y. Liu, J. H. Prestegard, A device for the measurement of residual chemical shift anisotropy and residual dipolar coupling in soluble and membrane-associated proteins, Journal of Biomolecular NMR 47 (2010) $249-258$.

[16] A. Bax, A. Grishaev, Weak alignment NMR: a hawk-eyed view of biomolecular structure, Current Opinion in Structural Biology 15 (5) (2005) 563-570.

[17] A. Naito, Structure elucidation of membrane-associated peptides and proteins in oriented bilayers by solid-state NMR spectroscopy, Solid State Nuclear Magnetic Resonance 36 (2009) 67-76. 
[18] P. Thureau, A. C. Sauerwein, M. Concistrè, M. H. Levitt, Selective internuclear coupling estimation in the solid-state NMR of multiplespin systems, Physical Chemistry, Chemical Physics 13 (2011) 93-96.

[19] A. Lange, S. Luca, M. Baldus, Structural constraints from protonmediated rare-spin correlation spectroscopy in rotating solids, Journal of the American Chemical Society 124 (33) (2002) 9704-9705.

[20] T. Manolikas, T. Herrmann, B. H. Meier, Protein structure determination from C-13 spin-diffusion solid-state NMR spectroscopy, Journal of the American Chemical Society 130 (12) (2008) 3959-3966.

[21] J. S. Waugh, Uncoupling of local field spectra in nuclear magnetic resonance: Determination of atomic positions in solids, Proceedings of the National Academy of Sciences of the United States of America 73 (5) (1976) 1394-1397.

[22] S. V. Dvinskikh, H. Zimmermann, A. Maliniak, D. Sandstrom, Measurements of motionally averaged heteronuclear dipolar couplings in MAS NMR using R-type recoupling, Journal of Magnetic Resonance 168 (2) (2004) 194-201.

[23] T. M. Ferreira, B. Medronho, R. W. Martin, D. Topgaard, Segmental order parameters in a nonionic surfactant lamellar phase studied with ${ }^{1} \mathrm{H}-{ }^{13} \mathrm{C}$ solid-state NMR, Physical Chemistry Chemical Physics 10 (2008) 6033-6038.

[24] A. Ramamoorthy, S. J. Opella, 2-dimensional chemical-shift heteronuclear dipolar coupling spectra obtained with Polarization Inversion Spin-Exchange at the Magic Angle and Magic-Angle Sample-Spinning (PISEMAMAS), Solid State Nuclear Magnetic Resonance 4 (6) (1995) 387-392.

[25] A. Ramamoorthy, C. H. Wu, S. J. Opella, 3-dimensional solid-state NMR experiment that correlates the chemical-shift and dipolar coupling frequencies of 2 heteronuclei, Journal Of Magnetic Resonance Series B 107 (1) (1995) 88-90.

[26] S. J. Opella, A. A. Nevzorov, Selective averaging for high-resolution solid-state NMR spectroscopy of aligned samples, Journal of Magnetic Resonance 185 (2007) 59-70. 
[27] S. V. Dvinskikh, K. Yamamoto, A. Ramamoorthy, Heteronuclear Isotropic Mixing Separated Local Field NMR Spectroscopy, Journal of Chemical Physics 125 (2006) 34507.

[28] T. Gopinath, R. Verardi, N. J. Traaseth, G. Veglia, Sensitivity enhancement of separated local field experiments: Application to membrane proteins, Journal of Physical Chemistry B 114 (2010) 5089-5095.

[29] T. Gopinath, K. R. Mote, G. Veglia, Sensitivity and resolution enhancement of oriented solid-state NMR: Application to membrane proteins, Progress in Nuclear Magnetic Resonance Spectroscopy 75 (2013) 50-68.

[30] G. J. Lu, S. J. Opella, Resonance assignments of a membrane protein in phospholipid bilayers by combining multiple strategies of oriented sample solid-state NMR, Journal of Biomolecular NMR 58 (2014) 6981.

[31] R. Mahalakshmi, F. M. Marassi, Orientation of the Escherichia coli outer membrane protein OmpX in phospholipid bilayer membranes determined by solid-state NMR, Biochemistry 47 (2008) 6531-6538.

[32] A. A. Nevzorov, R. W. Knox, G. J. Lu, S. J. Opella, A resonance assignment method for oriented-sample solid-state NMR of proteins, Journal of the American Chemical Society 132 (24) (2010) 8255-8257.

[33] B. B. Das, H. J. Nothnagel, G. J. Lu, W. S. Son, Y. Tian, F. M. Marassi, S. J. Opella, Structure determination of a membrane protein in proteoliposomes, Journal of the American Chemical Society 134 (2012) $2047-2056$.

[34] Y. Yin, A. A. Nevzorov, Structure determination in "shiftless" solid state NMR of oriented protein samples, Journal of Magnetic Resonance 212 (2011) $64-73$.

[35] L. Shi, N. J. Traaseth, R. Verardi, A. Cembran, J. Gao, G. Veglia, A refinement protocol to determine structure, topology, and depth of insertion of membrane proteins using hybrid solution and solid-state NMR restraints, Journal of Biomolecular NMR 44 (2009) 195-205. 
[36] H. Saitô, I. Ando, A. Ramamoorthy, Chemical shift tensor - the heart of NMR: Insights into biological aspects of proteins, Progress in Nuclear Magnetic Resonance Spectroscopy 57 (2010) 181-228.

[37] J. Herzfeld, A. E. Berger, Sideband intensities in NMR spectra of samples spinning at the magic angle, Journal of Chemical Physics 73 (1980) 6021-6030.

[38] P. Hodgkinson, L. Emsley, The reliability of the determination of tensor parameters by solid-state nuclear magnetic resonance, Journal of Chemical Physics 107 (1997) 4808-4816.

[39] W. Dixon, Spinning-sideband-free NMR spectra, Journal of Magnetic Resonance 44 (1981) 220-223.

[40] E. T. Olejniczak, S. Vega, R. G. Griffin, Multiple pulse NMR in rotating solids, Journal of Chemical Physics 81 (1984) 4804-4817.

[41] D. P. Raleigh, E. T. Olejniczak, S. Vega, R. G. Griffin, An analysis of sideband suppression techniques in magic-angle sample spinning NMR, Journal of Magnetic Resonance 72 (1987) 238-250.

[42] N. C. Nielsen, H. Bildsoe, H. J. Jakobsen, TOSS in high-speed MAS NMR, Journal of Magnetic Resonance 80 (1988) 9- I54.

[43] W. T. Dixon, Spinning-sideband-free and spinning-sideband-only NMR spectra in a spinning sample, Journal of Chemical Physics 77 (1982) 1800-1809.

[44] R. Tycko, G. Dabbagh, P. Mirau, Determination of chemical-shiftanisotropy lineshapes in a two-dimensional magic-angle-spinning NMR experiment, Journal of Magnetic Resonance 85 (1989) 265-274.

[45] S. F. Liu, J. D. Mao, K. Schmidt-Rohr, A robust technique for twodimensional separation of undistorted chemical-shift anisotropy powder patterns in magic-angle-spinning NMR, Journal of Magnetic Resonance 155 (2002) $15-28$.

[46] R. M. Orr, M. J. Duer, Recoupling of chemical-shift anisotropy powder patterns in MAS NMR, Journal of Magnetic Resonance 181 (1) (2006) $1-8$. 
[47] L. Shao, J. J. Titman, Chemical shift anisotropy amplification, Progress in Nuclear Magnetic Resonance Spectroscopy 51 (2007) 103137.

[48] M. Carravetta, M. Eden, X. Zhao, A. Brinkmann, M. H. Levitt, Symmetry principles for the design of radiofrequency pulse sequences in the nuclear magnetic resonance of rotating solids, Chemical Physics Letters 321 (2000) 205-215.

[49] G. Hou, I. Byeon, J. Ahn, A. M. Gronenborn, T. Polenova, Recoupling of chemical shift anisotropy by R-symmetry sequences in magic angle spinning NMR spectroscopy, Journal of Chemical Physics 137 (13) (2012) 134-201.

[50] M. K. Pandey, M. Malon, A. Ramamoorthy, Y. Nishiyama, Composite$180^{\circ}$ pulse-based symmetry sequences to recouple proton chemical shift anisotropy tensors under ultrafast MAS solid-state NMR spectroscopy, Journal of Magnetic Resonance 250 (2015) 45-54.

[51] G. Hou, S. Paramasivam, I. L. Byeon, A. M. Gronenborn, T. Polenova, Determination of relative tensor orientations by $\gamma$-encoded chemical shift anisotropy/heteronuclear dipolar coupling 3D NMR spectroscopy in biological solids, Physical Chemistry Chemical Physics 12 (2010) $14873-14883$.

[52] J. C. C. Chan, R. Tycko, Recoupling of chemical shift anisotropies in solid-state nmr under high-speed magic-angle spinning and in uniformly c-13- labeled systems, Journal of Chemical Physics 118 (18) (2003) 8378-8389.

[53] Y. Nishiyama, T. Yamazaki, T. Terao, Development of modulated rf sequences for decoupling and recoupling of nuclear-spin interactions in sample-spinning solid-state NMR: application to chemicalshift anisotropy determination, Journal of Chemical Physics 124 (2006) 064304.

[54] B. J. Wylie, C. D. Schwieters, E. Oldfield, C. M. Rienstra, Protein structure refinement using ${ }^{13} \mathrm{C}_{\alpha}$ chemical shift tensors, Journal of the American Chemical Society 131 (2009) 985-992. 
[55] B. J. Wylie, L. J. Sperling, A. J. Nieuwkoop, W. T. Franks, E. Oldfield, C. M. Rienstra, Ultrahigh resolution protein structures using NMR chemical shift tensors, Proceedings of the National Academy of Sciences of the United States of America 108 (41) (2011) 16974-16979.

[56] Y. Tian, G. J. Lu, F. M. Marassi, S. J. Opella, Structure of the membrane protein MerF, a bacterial mercury transporter, improved by the inclusion of chemical shift anisotropy constraints, Journal of Biomolecular NMR 60 (2014) 67-71.

[57] N. K. Sethi, D. M. Grant, R. J. Pugmire, C-13 chemical shielding anisotropy studied by variable-angle sample spinning, Journal of Magnetic Resonance 71 (3) (1987) 476-479.

[58] E. O. Stejskal, J. Schaefer, R. A. McKay, High-resolution, slow-spinning magic-angle carbon-13 NMR, Journal of Magnetic Resonance 25 (1977) 569.

[59] T. Wang, M. Hong, Investigation of the curvature induction and membrane localization of the influenza virus M2 protein using static and off-magic-angle spinning solid-state nuclear magnetic resonance of oriented bicelles, Biochemistry 54 (2015) 2214-2226.

[60] A. Bax, N. Szeverenyi, G. Maciel, Chemical-shift anisotropy in powdered solids studied by 2D FT CP MAS NMR, Journal of Magnetic Resonance 51 (1983) 400-408.

[61] O. N. Antzutkin, S. C. Shekar, M. H. Levitt, Two-dimensional sideband separation in magic-angle-spinning NMR, Journal of Magnetic Resonance A 115 (1995) 7-19.

[62] A. Bax, N. Szeverenyi, G. E. Maciel, Correlation of isotropic shifts and chemical shift anisotropies by two-dimensional Fourier-transform magic-angle hopping NMR spectroscopy, Journal of Magnetic Resonance 52 (1983) 147-152.

[63] Z. Gan, High-resolution chemical shift and chemical shift anisotropy correlation in solids using slow magic angle spinning, Journal of the Americal Chemical Society 114 (1992) 8307-8309. 
[64] J. Z. Hu, D. W. Alderman, C. H. Ye, R. J. Pugmire, D. M. Grant, An isotropic chemical shift-chemical shift anisotropy magic-angle slowspinning 2D NMR experiment, Journal of Magnetic Resonance A 105 (1993) 82-87.

[65] Z. H. Gan, R. R. Ernst, An improved 2D magic-angle-turning pulse sequence for the measurement of chemical-shift anisotropy, Journal of Magnetic Resonance A 123 (1996) 140-143.

[66] M. Strohmeier, D. M. Grant, A new sensitive isotropic-anisotropic separation experiment - SPEED MAS, Journal of Magnetic Resonance 168 (2004) 296-306.

[67] J. Z. Hu, R. A. Wind, The evaluation of different MAS techniques at low spinning rates in aqueous samples and in the presence of magnetic susceptibility gradients, Journal of Magnetic Resonance 159 (2002) $92-100$.

[68] Y. Hu, E. Levin, K. Schmidt-Rohr, Broadband "infinite-speed" magicangle spinning NMR spectroscopy, Journal of the Americal Chemical Society 131 (2009) 8390-8391.

[69] I. Hung, Z. Gan, On the magic-angle turning and phase-adjusted spinning sidebands experiments, Journal of Magnetic Resonance 204 (2010) $150-154$.

[70] C. Crockford, H. Geen, J. Titman, Two-dimensional MAS-NMR spectra which correlate fast and slow magic-angle spinning sideband patterns, Chemical Physics Letters 344 (2001) 367-373.

[71] L. Shao, C. Crockford, H. Geen, G. Grasso, J. Titman, Chemical shift anisotropy amplification, Journal of Magnetic Resonance 167 (2004) $75-86$.

[72] R. M. Orr, M. J. Duer, S. E. Ashbrook, Correlating fast and slow chemical shift spinning sideband patterns in solid-state NMR, Journal of Magnetic Resonance 174 (2005) 301-309.

[73] Z. H. Gan, D. M. Grant, Molecular and structural information from variable-angle spinnig NMR dipolar spectra of C-13-N-14 systems, Journal of Magnetic Resonance 90 (3) (1990) 522-534. 
[74] L. J. Mueller, Tensors and rotations in NMR, Concepts in Magnetic Resonance Part A 38A (5) (2011) 221-235.

[75] S. Ganapathy, S. Schramm, E. Oldfield, Variable-angle sample-spinning high-resolution NMR of solids, Journal of Chemical Physics 77 (9) (1982) 4360-4365.

[76] M. Goldman, P. J. Grandinetti, A. Llor, Z. Olejniczak, J. R. Sachleben, J. W. Zwanziger, tical aspects of higher-order truncations in solid-state nuclear magnetic resonance, The Journal of Chemical Physics 97 (1992) $8947-8960$.

[77] N. K. Sethi, D. W. Alderman, D. M. Grant, NMR spectra from powdered solids spinning at any angle and speed: simulations and experiments, Molecular Physics 71 (2) (1990) 17.

[78] Z. W. Zheng, Z. H. Gan, N. K. Sethi, D. W. Alderman, D. M. Grant, An efficient simulation of variable-angle spinning lineshapes for the quadrupolar nuclei with half-integer spin, Journal of Magnetic Resonance 95 (3) (1991) 509-522.

[79] T. Chen, J. Wang, H. X. Li, K. X. Wang, F. Deng, Y. R. Du, D. T. Ding, Sodium cation locations in zeolite omega studied by ${ }^{23}$ na VASS NMR, Chemical Physics Letters 238 (1995) 82-87.

[80] L. Frydman, G. Chingas, Y. Lee, P. Grandinetti, M. Eastman, G. A. Barrall, A. Pines, Variable-angle correlation spectroscopy in solid-state nuclear magnetic resonance, Journal of Chemical Physics 97 (7) (1992) 4800-4808.

[81] G. Cory, J. B. Miller, A. N. Garroway, W. S. Veeman, Chemical-shiftresolved back-projection imaging, Journal of Magnetic Resonance 85 (1969) 219-227.

[82] L. Frydman, Y. K. Lee, L. Emsley, G. C. Chingas, A. Pines, Variableangle three-dimensional exchange nuclear magnetic resonance spectroscopy for the study of molecular motion in complex solids, Journal of the American Chemical Society 115 (1993) 4825.

[83] Y. K. Lee, L. Emsley, R. G. Larsen, K. Schmidt-Rohr, M. Hong, L. Frydman, G. C. Chingas, A. Pines, Three-dimensional variable-angle 
nuclear magnetic resonance exchange spectroscopy without rotor axis hopping, The Journal of Chemical Physics 101 (3) (1994) 1852-1864.

[84] A. Medek, J. R. Sachleben, P. Beverwyk, L. Frydman, Multi-rank nuclear magnetic resonance studies of half-integer quadrupolar nuclei in solids by three-dimensional dynamic-angle correlation spectroscopy, Journal of Chemical Physics 104 (14) (1996) 5374-5383.

[85] J. P. Amoreux, variable-angle double rotation technique: a new twodimensional high-resolution technique, Solid State Nuclear Magnetic Resonance 4 (229-239).

[86] T. Gullion, R. A. McKay, A. Schmidt, Spin-echo, double-resonance NMR with flipped spinning (SEDORFS), Journal of Magnetic Resonance 94 (1991) 362-369.

[87] A. Pines, J. S. Waugh, M. G. Gibby, Proton-enhanced nuclear induction spectroscopy - method for high-resolution NMR of dilute spins in solids, Journal of Chemical Physics 56 (4) (1972) 1776.

[88] J. Schaefer, E. O. Stejskal, C-13 nuclear magnetic resonance of polymers spinning at magic angle, Journal of the American Chemical Society 98 (4) (1976) 1031-1032.

[89] X. L. Wu, K. W. Zilm, Cross polarization with high-speed magic-angle spinning, Journal of Magnetic Resonance, Series A 104 (2) (1993) 154165.

[90] E. Andrew, Magic angle spinning in solid-state NMR spectroscopy, Philosophical Transactions of the Royal Society of London Series A -Mathematical Physical and Engineering Sciences 299 (1981) 505-520.

[91] M. Sardashti, G. E. Maciel, Effects of sample spinning on cross polarization, Journal of Magnetic Resonance 72 (1987) 467-474.

[92] G. Zandomeneghi, B. H. Meier, Adiabatic-passage cross polarization in N-15 NMR spectroscopy of peptides weakly associated to phospholipids: Determination of large RDC, Journal of Biomolecular NMR 30 (3) (2004) 303-309. 
[93] A. Böckmann, M. Ernst, B. H. Meier, Spinning proteins, the faster, the better?, Journal of Magnetic Resonance 253 (2015) 71-79.

[94] R. C. Zeigler, R. A. Wind, G. E. Maciel, The stop-and-go spinning technique in MAS experiments, Journal of Magnetic Resonance 79 (2) (1988) 299-306.

[95] S. Laage, A. Marchetti, J. Sein, R. Pieratelli, H. Sass, S. Grzesiek, A. Lesage, G. Pintacuda, L. Emsley, Band-selective ${ }^{1} \mathrm{H}-{ }^{13} \mathrm{C}$ crosspolarization in fast magic angle spinning solid-state NMR spectroscopy, Journal of the American Chemical Society 130 (2008) 17216-17217.

[96] A. Lange, I. Scholz, T. Manolikas, M. Ernst, B. H. Meier, Lowpower cross polarization in fast magic-angle spinning NMR experiments, Chemical Physics Letters 468 (1-3) (2009) 100-105.

[97] M. Ernst, A. Samoson, B. H. Meier, Low-power decoupling in fast magic-angle spinning NMR, Chemical Physics Letters 348 (2001) 293302.

[98] M. Ernst, A. Samoson, B. H. Meier, Low-power XiX decoupling in MAS NMR experiments, Journal of Magnetic Resonance 163 (2) (2003) 332339.

[99] M. Kotecha, N. P. Wickramasinghe, Y. Ishii, Efficient low-power heteronuclear decoupling in C-13 high-resolution solid-state NMR under fast magic angle spinning, Magnetic Resonance in Chemistry 45 (2007) S221-S230.

[100] P. D. Murphy, B. C. Gerstein, Principal components of the cadmium113 shielding tensors in cadmium sulfate hydrates: a nuclear magnetic resonance study of cadmium coordinated with oxygen, Journal of the Americal Chemical Society 103 (1981) 3282-3286.

[101] A. M. Orendt, N. K. Sethi, J. C. Facelli, W. J. Horton, R. J. Pugmire, D. M. Grant, Carbon-13 chemical shift tensors in aromatic compounds. 4. substituted naphthalenes, Journal of the American Chemical Society 114 (1992) 2832-2836.

[102] R. G. Larsen, Y. K. Lee, B. He, J. O. Yang, Z. Luz, H. Zimmermann, A. Pines, C-13 chemical-shift tensor correlation via spin-diffusion in 
solid tropolone using switched-angle spinning spectroscopy, Journal of Chemical Physics 103 (22) (1995) 9844-9854.

[103] M. Mobli, M. W. Maciejewski, A. D. Schuyler, A. S. Sternc, J. C. Hoch, Sparse sampling methods in multidimensional NMR, Physical Chemistry Chemical Physics 14 (2012) 10835-10843.

[104] S. G. Hyberts, A. G. Milbradt, A. B. Wagner, H. Arthanari, G. Wagner, Application of iterative soft thresholding for fast reconstruction of NMR data non-uniformly sampled with multidimensional Poisson Gap scheduling, Journal of Biomolecular NMR 52 (4) (2012) 315-327.

[105] K. W. Zilm, D. M. Grant, Carbon-13 dipolar spectroscopy of small organic molecules in argon matrixes, Journal of the American Chemical Society 103 (1981) 2913-2922.

[106] B. H. Suits, J. Sepa, D. White, Simulation of slow Variable-Angle Spinning NMR Spectra. application to ${ }^{13} \mathrm{C}$ bonded to N, Journal of Magnetic Resonance, Series A 120 (1) (1996) 88-96.

[107] D. Sakellariou, C. A. Meriles, R. W. Martin, A. Pines, High-resolution nmr of anisotropic samples with spinning away from the magic angle, Chemical Physics Letters 377 (3-4) (2003) 333-339.

[108] R. W. Martin, R. C. Jachmann, D. Sakellariou, U. G. Nielsen, A. Pines, High-resolution NMR spectroscopy of biological tissues using projected magic angle spinning, Magnetic Resonance in Medicine 54 (2005) 253257.

[109] D. J. Philp, W. A. Bubb, P. W. Kuchel, Chemical shift and magnetic susceptibility contributions to the separation of intracellular and supernatant resonances in Variable Angle Spinning NMR spectra of erythrocyte suspensions, Magnetic Resonance in Medicine 51 (2004) 441-444.

[110] T. J. Larkin, W. A. Bubb, P. W. Kuchel, Water chemical shift in 1H NMR of red cells: Effects of $\mathrm{pH}$ when transmembrane magnetic susceptibility differences are low, Magnetic Resonance in Medicine 59 (2008) 707-711. 
[111] A. C. Kolbert, P. J. Grandinetti, M. Baldwin, S. B. Prusiner, A. Pines, Measurement of internuclear distances by Switched Angle Spinning NMR, Journal of Physical Chemistry 98 (1994) 7936-7936.

[112] R. Tycko, Normal angle spinning dipolar spectroscopy for structural studies by solid-state nuclear magnetic resonance, Journal of the American Chemical Society 116 (5) (1994) 2217-2218.

[113] A. Bax, N. Szeverenyi, G. Maciel, Chemical shift anisotropy in powdered solids studied by 2D FT NMR with flipping of the spinning axis, Journal of Magnetic Resonance 55 (1983) 494-497.

[114] J. Iwamiya, M. F. Davis, G. Maciel, A 1D FT experiment for obtaining chemical-shift tensors in complex solids, using selective excitation and flipping of the spinner axis, Journal of Magnetic Resonance 88 (1990) 199-204.

[115] G. E. Maciel, N. Szeverenyi, M. Sardashti, chemical-shift-anisotropy powder patterns by the two-dimensional angle-flipping approach. Effects of crystallite packing, Journal of Magnetic Resonance 64 (1985) 365-374.

[116] P. Zhang, C. Dunlap, P. Florian, P. J. Grandinetti, I. Farnan, J. F. Stebbins, Silicon site distributions in an alkali silicate glass derived by two-dimensional ${ }^{29} \mathrm{Si}$ nuclear magnetic resonance, Journal of NonCrystalline Solids 204 (1996) 294-300.

[117] P. Zhang, P. J. Grandinetti, J. F. Stebbins, Anionic species determination in $\mathrm{CaSiO}_{3}$ glass using two-dimensional ${ }^{29} \mathrm{Si} \mathrm{NMR}$, Journal of Physical Chemistry B 101 (1997) 4004-4008.

[118] M. Davis, D. Kaseman, S. M. Parvani, K. Sanders, P. Grandinetti, D. Massiot, P. Florian, Q((n)) species distribution in K2O center dot 2SiO(2) glass by Si-29 Magic Angle Flipping NMR, Journal of Physical Chemistry A 114 (17) (2010) 5503-5508.

[119] M. Davis, K. Sanders, P. Grandinetti, S. Gaudio, S. Sen, Structural investigations of magnesium silicate glasses by Si-29 2D Magic-Angle Flipping NMR, Journal of Non-Crystalline Solids 357 (15) (2011) 27872795. 
[120] D. Hoult, R. Richards, The signal-to-noise ratio of the nuclear magnetic resonance experiment, Journal of Magnetic Resonance 24 (1976) 71-85.

[121] C. Qian, P. Thureau, R. Martin, Variable angle spinning (VAS) experiments for strongly oriented systems: Methods development and preliminary results, Magnetic Resonance in Chemistry 46 (2008) 351355.

[122] S. Idziak, U. Haeberlen, Design and construction of a high homogeneity rf coil for solid-state multiple-pulse nmr, Journal of Magnetic Resonance 50 (2) (1982) 281-288, times Cited: 41.

[123] Y. Sun, G. E. Maciel, The tilted coil for NMR experiments, Journal of Magnetic Resonance Series A 105 (1993) 145-150.

[124] J. Alonso, A. Soleilhavoup, A. Wong, A. Guiga, D. Sakellariou, Double helix dipole design applied to magnetic resonance: A novel NMR coil, Journal of Magnetic Resonance 235 (2013) 32-41.

[125] J. A. Stringer, C. Bronniman, C. Mullen, D. Zhou, S. Stellfox, Y. Li, E. Williams, C. Rienstra, Reduction of rf-induced sample heating with a scroll coil resonator structure for solid-state nmr probes, Journal of Magnetic Resonance 173 (2005) 40-48.

[126] B. Dillmann, K. Elbayed, H. Zeiger, M.-C. Weingertner, M. Piotto, F. Engelke, A novel low-E field coil to minimize heating of biological samples in solid-state multinuclear NMR experiments, Journal of Magnetic Resonance 187 (2007) 10-18.

[127] T. G. Ajithkumar, E. R. H. van Eck, A. P. M. Kentgens, Homonuclear correlation experiments for quadrupolar nuclei, spinning away from the magic angle, Solid State Nuclear Magnetic Resonance 26 (3-4) (2004) 180-186.

[128] I. M. Litvak, C. A. Espinosa, R. A. Shapiro, A. N. Oldham, V. V. Duong, R. W. Martin, Pneumatic switched angle spinning NMR probe with capacitively coupled double saddle coil, Journal of Magnetic Resonance 206 (2010) 183-189. 
[129] D. Alderman, D. Grant, Efficient decoupler coil design which reduces heating in conductive samples in superconducting spectrometers, Journal of Magnetic Resonance 36 (3) (1979) 447-451.

[130] C. V. Grant, Y. Yang, M. Glibowicka, C. H. Wu, S. H. Park, C. Deber, S. Opella, A modified Alderman-Grant coil makes possible an efficient cross-coil probe for high field solid-state NMR of lossy biological samples, Journal of Magnetic Resonance 201 (2009) 87-92.

[131] P. Gor'kov, S. A. McNeill, K. Shetty, W. W. Brey, J. R. Long, A lowE Magic Angle Spinning probe for biological solid-state NMR at 750 MHz, Journal of Magnetic Resonance 197 (2009) 135-144.

[132] F. D. Doty, J. Kulkarni, C. Turner, G. Entzminger, A. Bielecki, Using a cross-coil to reduce RF heating by an order of magnitude in triple-resonance multinuclear MAS at high fields, Journal of Magnetic Resonance 182 (2) (2006) 239-253.

[133] B. C. Gerstein, H. J. Pan, M. Pruski, Stable system for multiple pulse, magic angle spinning and dynamic angle spinning NMR, Review of Scientific Instruments 61 (10) (1990) 2699-2701.

[134] K. T. Mueller, G. C. Chingas, A. Pines, NMR probe for dynamic-angle spinning, Review of Scientific Instruments 62 (6) (1991) 1445-1452.

[135] F. Doty, G. Entzminger, Y. A. Yang, Magnetism in high-resolution NMR probe design. ii: HR MAS, Concepts in Magnetic Resonance 10 (4) (1998) 239-260.

[136] M. A. Eastman, P. J. Grandinetti, Y. K. Lee, A. Pines, Double-tuned hopping-coil probe for dynamic-angle-spinning NMR, Journal of Magnetic Resonance 98 (2) (1992) 333-341.

[137] K. T. Mueller, B. Q. Sun, G. C. Chingas, J. W. Zwanziger, T. Terao, A. Pines, Dynamic-angle spinning of quadrupolar nuclei, Journal of magnetic resonance 86 (1990) 470-487.

[138] F. Doty, High power flexible leads for DAS NMR, U.S. Patent 6,198,284 (2001). 
[139] F. D. Doty, P. D. Ellis, Design of high speed cylindrical NMR sample spinners, Review of Scientific Instruments 52 (1981) 1868.

[140] R. A. McKay, Transmission Line Probes, Vol. 6 of Encyclopedia of Nuclear Magnetic Resonance, Wiley, Chichester, 1996, pp. 3768 - 3771.

[141] R. W. Martin, E. Paulson, K. W. Zilm, Design of a triple resonance magic angle sample spinning probe for high field solid state nuclear magnetic resonance, Review of Scientific Instruments 74 (6) (2003) 3045-3061.

[142] E. R. Mihaliuk, A switched angle sample spinning probe and its application to solid state dipolar recoupling experiments, Presented at the 50th ENC Conference held in Monterey, CA, USA (March, 29 - April, $32009)$.

[143] K. T. Mueller, G. C. Chingas, A. Pines, NMR probe for dynamic-angle spinning, Review of Scientific Instruments 62 (6) (1991) 1445-1452.

[144] L. Chopin, R. Rosanke, T. Gullion, Simple improvements in spinningspeed control for MAS NMR experiments, Journal of Magnetic Resonance Series A 122 (1996) 237-239.

[145] J. N. Lee, D. W. Alderman, J. Y. Jin, K. W. Zilm, C. L. Mayne, R. Pugmire, D. M. Grant, Cylindrical spinner and speed controller for magic angle spinning nuclear magnetic resonance, Review of Scientific Instruments 55 (1984) 516-520.

[146] E. Hughes, T. Gullion, A simple, inexpensive, and precise magic angle spinning speed controller, Solid State Nuclear Magnetic Resonance 26 (2004) 16-21.

[147] E. R. Mihaliuk, T. Gullion, Using heat to control the sample spinning speed in MAS NMR, Journal of Magnetic Resonance 212 (2011) 249253.

[148] R. A. Wind, F. E. Anthonio, M. J. Duijvestijn, J. Smidt, J. Trommel, G. M. C. DeVette, Experimental setup for enhanced ${ }^{13} \mathrm{C} \mathrm{nmr}$ spectroscopy in solids using dynamic nuclear polarization, Journal of Magnetic Resonance 52 (1983) 424. 
[149] S. F. Dec, R. A. Wind, G. E. Maciel, F. E. Anthonio, High-speed magic-angle spinning, Journal of Magnetic Resonance 70 (1986) 355.

[150] J. Courtieu, D. W. Alderman, D. M. Grant, Spinning near the magic angle: A means of obtaining first-order dipolar NMR spectra of molecules dissolved in nematic liquid crystals, Journal of the Americal Chemical Society 103 (1981) 6783-6784.

[151] J. Courtieu, D. W. Alderman, D. M. Grant, J. P. Bayle, Director dynamics and NMR applications of nematic liquid crystals spinning at various angles from the magnetic field, Journal of Chemical Physics 77 (1982) 723 .

[152] T. Väänänen, J. Jokisaari, M. Selantaus, A variable-angle spinning system for the determination of NMR parameters of liquid-crystalline samples, Journal of Magnetic Resonance 72 (3) (1987) 414-421.

[153] M. Tomaselli, B. H. Meier, M. Baldus, J. Eisenegger, R. R. Ernst, An rf-driven nuclear spin-diffusion experiment using zero-angle sample spinning, Chemical Physics Letters 225 (1-3) (1994) 131-139.

[154] C. Qian, A. Pines, R. W. Martin, Design and construction of a contactless mobile RF coil for double resonance variable angle spinning NMR, Journal of Magnetic Resonance 188 (1) (2007) 183-189.

[155] J. Courtieu, J. P. Bayle, B. M. Fung, Variable angle sample spinning NMR in liquid crystals, Progress in Nuclear Magnetic Resonance Spectroscopy 26 (Part 2) (1994) 141-169.

[156] L. Beguin, J. Courtieu, L. Ziani, D. Merlet, Simplification of the H-1 NMR spectra of enantiomers dissolved in chiral liquid crystals, combining variable angle sample spinning and selective refocusing experiments, Magnetic Resonance in Chemistry 44 (12) (2006) 1096-1101.

[157] T. Väänänen, J. Jokisaari, M. Seläntaus, A variable-angle spinning system for the determination of NMR parameters of liquid-crystalline samples, Journal of Magnetic Resonance 72 (3) (1987) 414-421.

[158] E. R. Mihaliuk, T. Gullion, Optical lever for monitoring of the magic angle, Journal of Magnetic Resonance 223 (2012) 46-50. 
[159] G. Zandomeneghi, M. Tomaselli, J. D. van Beek, B. H. Meier, Manipulation of the director in bicellar mesophases by sample spinning: A new tool for NMR spectroscopy, Journal of the American Chemical Society 123 (5) (2001) 910-913.

[160] F. D. Doty, The Encyclopedia of NMR, Vol. 7, Wiley, 1996, Ch. Solid State NMR Probe Design, pp. 4475-4485.

[161] P. L. Gor'kov, R. Witter, E. Y. Chekmenev, F. Nozirov, R. Fu, W. W. Brey, Low-E probe for F-19-H-1 NMR of dilute biological solids, Journal of Magnetic Resonance 189 (2) (2007) 182-189.

[162] P. L. Gor'kov, E. Y. Chekmenev, C. G. Li, M. Cotten, J. J. Buffy, N. J. Traaseth, G. Veglia, W. W. Brey, Using low-E resonators to reduce RF heating in biological samples for static solid-state NMR up to $900 \mathrm{MHz}$, Journal of Magnetic Resonance 185 (1) (2007) 77-93.

[163] E. Paulson, R. W. Martin, K. W. Zilm, Cross polarization, radio frequency field homogeneity, and circuit balancing in high field solid state nmr probes, Journal of Magnetic Resonance 171 (2004) 314-323.

[164] F. Doty, G. Entzminger, Y. A. Yang, Magnetism in high-resolution NMR probe design. i: General methods, Concepts in Magnetic Resonance 10 (3) (1998) 133-156.

[165] S. Mamone, A. Dorsch, O. G. Johannessen, M. V. Naik, P. K. Madhu, M. Levitt, A Hall effect angle detector for solid-state NMR, Journal of Magnetic Resonance 190 (1) (2008) 135-141.

[166] J. S. Frye, G. S. Maciel, Setting the magic angle using a quadrupolar nuclide, Journal of Magnetic Resonance 48 (1982) 125-131.

[167] M. Tomaselli, B. H. Meier, M. Baldus, J. Eisenegger, R. Ernst, An rfdriven nuclear spin-diffusion experiment using zero-angle sample spinning, Chem. Phys. Lett. 225 (1994) 131-139.

[168] B. F. Chmelka, K. T. Mueller, A. Pines, J. Stebbins, Y. Wu, J. W. Zwanziger, ${ }^{17} \mathrm{O}$ NMR in solids by dynamic-angle spinning and double rotation, Nature 339 (6219) (1989) 42-43. 
[169] J. H. Baltisberger, S. L. Gann, P. J. Grandinetti, A. Pines, Cross polarization dynamic-angle spinning nuclear-magnetic resonance of quadrupole nuclei, Molecular Physics 81 (5) (1994) 1109-1124.

[170] P. J. Grandinetti, Y. K. Lee, J. H. Baltisberger, B. Q. Sun, A. Pines, Side-band patterns in dynamic angle spinning NMR, Journal of Magnetic Resonance Series A 102 (2) (1993) 195-204.

[171] Y. Wu, Z. Peng, Z. Olejniczak, B. Q. Sun, A. Pines, Effects of double rotation on homonuclear spin systems, Journal of Magnetic Resonance Series A 102 (1993) 29-33.

[172] A. Llor, J. Virlet, Towards high-resolution NMR of more nuclei in solids: sample spinning with time-dependent spinner axis angle, Chemical Physics Letters 152 (2,3) (1988) 248-253.

[173] A. Samoson, E. Lippmaa, A. Pines, High resolution solid-state nmr, Molecular Physics 65 (1988) 1013.

[174] Y. Wu, B. Q. Sun, A. Pines, A. Samoson, E. Lipmaa, NMR experiments with a new double rotor, Journal of Magnetic Resonance 89 (1990) 297309.

[175] A. P. Howes, T. Anupold, V. Lemaitre, A. Kukol, A. Watts, A. Samoson, M. E. Smith, R. Dupree, Enhancing resolution and sensitivity of ${ }^{17} \mathrm{O}$ solid-state NMR through combining double rotation, ${ }^{1} \mathrm{H}$ decoupling and satellite modulation for biomolecular applications, Chemical Physics Letters 421 (2006) 42.

[176] A. P. M. Kentgens, E. R. H. van Eck, T. G. Ajithkumar, T. Anupold, J. Past, A. Reinhold, A. Samoson, New opportunities for double rotation NMR of half-integer quadrupolar nuclei, Journal of Magnetic Resonance 178 (2006) 212.

[177] K. Takegoshi, K. Hikichi, Effects of sample spinning on "overtone" NMR, Chemical Physics Letters 194 (4,5,6) (1992) 359-362.

[178] L. Frydman, J. S. Harwood, isotropic spectra of half-integer quadrupolar spins from bidimensional Magic Angle Spinning NMR, Journal of the American Chemical Society 117 (19) (1995) 5367-5368. 
[179] Z. H. Gan, sotropic NMR spectra of half-integer qadrupolar nuclei using satellite transitions and magic-angle spinning, Journal of the American Chemical Society 122 (2000) 3242-3243.

[180] S. E. Ashbrook, S. Wimperis, Satellite-transition MAS NMR of spin I $=3 / 2,5 / 2,7 / 2$, and $9 / 2$ nuclei: Sensitivity, resolution, and practical implementation, Journal of Magnetic Resonance 156 (2002) 269-281.

[181] J. T. Ash, N. M. Trease, P. J. Grandinetti, Separating chemical shift and quadrupolar anisotropies via multiple-quantum NMR spectroscopy, Journal of the American Chemical Society 30 (2008) 1085810859.

[182] A. Wong, A. P. Howes, B. Parkinson, T. Anupold, A. Samoson, D. Holland, R. Dupree, High-resolution ${ }^{17} \mathrm{O}$ double-rotation NMR characterization of ring and non-ring oxygen in vitreous $\mathrm{B}_{2} \mathrm{O}_{3}$, Physical Chemistry, Chemical Physics 11 (2009) 7061-7068.

[183] I. Hung, A. P. Howes, B. G. Parkinson, T. Anupold, A. Samoson, S. P. Brown, P. F. Harrison, D. Holland, R. Dupree, Determination of the bond-angle distribution in vitreous $\mathrm{B} 2 \mathrm{O} 3$ by ${ }^{11} \mathrm{~B}$ double rotation (DOR) NMR spectroscopy, Journal of Solid State Chemistry 182 (2009) 2402-2408.

[184] I. Hung, A. Wong, A. P. Howes, T. Anupold, A. Samoson, M. E. Smith, D. Holland, S. P. Brown, R. Dupree, Separation of isotropic chemical and second-order quadrupolar shifts by multiple-quantum double rotation NMR, Journal of Magnetic Resonance 197 (2009) 229.

[185] F. A. Perras, D. L. Bryce, Measuring dipolar and J coupling between quadrupolar nuclei using double-rotation NMR, The Journal of Chemical Physics 138 (2013) 174202.

[186] S. Wi, L. Frydman, Residual dipolar couplings between quadrupolar nuclei in high resolution solid state NMR: Description and observations in the high-field limit, The Journal of Chemical Physics 112 (2000) $3248-3261$.

[187] T. M. Clark, P. J. Grandinetti, P. Florian, J. F. Stebbins, Correlated structural distributions in silica glass, Physical Review B 70 (2004) 064202 . 
[188] Z. Yao, H. Kwak, D. Sakellariou, L. Emsley, P. J. Grandinetti, Sensitivity enhancement of the central transition NMR signal of quadrupolar nuclei under magic-angle spinning, Chemical Physics Letters 327 (2000) 85.

[189] S. Prasad, H. T. Kwak, T. Clark, P. J. Grandinetti, A simple technique for determining nuclear quadrupole coupling constants with RAPT solid-state NMR spectroscopy, Journal of the American Chemical Society 124 (2002) 4964.

[190] P. Hartmann, C. Jager, J. Zwanziger, Off-angle correlation spectroscopy applied to spin-1/2 and quadrupolar nuclei, Solid State Nuclear Magnetic Resonance 13 (1999) 245-254.

[191] C. Joo, U. Werner-Zwanziger, J. W. Zwanziger, Anisotropy-correlated spectroscopy of quadrupolar nuclei, Solid State Nuclear Magnetic Resonance 16 (2000) 77-83.

[192] U. Haeberlen, High Resolution NMR in Solids Selective Averaging: Supplement 1 Advances in Magnetic Resonance, Vol. 1 of Advances in Magnetic Resonance, Academic Press Inc., New York, 1976.

[193] R. K. Harris, R. E. Wasylishen, M. J. Duer, NMR Crystallography, John Wiley \& Sons, The Atrium, Southern Gate, West Sussex, PO19 8SQ Chichester, United Kingdom, 2009.

[194] M. M. Maricq, J. S. Waugh, NMR in rotating solids, Journal of Chemical Physics 70 (1979) 3300-3316.

[195] G. Wu, R. E. Wasylishen, Spinning-frequency-dependent ${ }^{31} \mathrm{P}$ MAS NMR spectra of square-planar metal-phosphine complexes, Inorganic Chemistry 33 (1994) 2114-2718.

[196] A. P. S. Jurd, J. J. Titman, Measurements of relative chemical shift tensor orientations in solid-state NMR: new slow magic angle spinning dipolar recoupling experiments, Physical Chemistry Chemical Physics 11 (2009) 6999-7007.

[197] G. Wu, R. E. Wasylishen, Observation of ${ }^{31} \mathrm{P}_{-}{ }^{31} \mathrm{P}$ indirect spin-spin coupling in copper-bis(phosphine) complexes by two-dimensional solidstate NMR, Inorganic Chemistry 35 (1996) 3113-3116. 
[198] K. Eichele, G. C. Ossenkamp, R. E. Wasylishen, T. S. Cameron, Phosphorus-31 solid-state NMR studies of homonuclear spin pairs in molybdenum phosphine complexes: Single-crystal, dipolar-chemical shift, rotational-resonance, and 2D spin-echo NMR experiments, Inorganic Chemistry 38 (1999) 639-651.

[199] T. Vosegaard, E. Hald, V. Langer, H. J. Skov, P. Daugaard, H. Bildsøe, H. J. Jakobsen, Improved hardware and software for single-crystal NMR spectroscopy, Journal of Magnetic Resonance 135 (1998) 126132.

[200] P. J. Grandinetti, Does phase cycling work for nuclei experiencing strong quadrupolar couplings?, Solid State Nuclear Magnetic Resonance 23 (2003) 1-13.

[201] N. M. Trease, P. J. Grandinetti, Solid-state nuclear magnetic resonance in the rotating tilted frame, The Journal of Chemical Physics 128 (2008) 052318.

[202] P. J. Grandinetti, J. T. Ash, N. M. Trease, Symmetry pathways in solidstate NMR, Progress in Nuclear Magnetic Resonance Spectroscopy 59 (2011) 121-196.

[203] G. Wu, R. E. Wasylishen, Direct determination of J coupling between 2 crystallographically equivalent spins from 2-dimensional Jresolved nuclear-magnetic-resonance spectra under Variable-AngleSpinning conditions, Journal of Chemical Physics 100 (8) (1994) 55465549.

[204] G. Wu, R. E. Wasylishen, Variable-Angle-Spinning nuclear-magneticresonance spectra of homonuclear spin pairs consisting of 2 crystallographically equivalent spins in the solid-state, Journal of Chemical Physics 100 (7) (1994) 4828-4834.

[205] T. Mizuno, K. Takegoshi, T. Terao, Switching-angle sample spinning NMR probe with a commercially available $20 \mathrm{kHz}$ spinning system, Journal of Magnetic Resonance 171 (1) (2004) 15-19.

[206] A. Bax, G. Kontaxis, N. Tjandra, Dipolar couplings in macromolecular structure determination, Methods in Enzymology 339 (2001) 127-174. 
[207] G. Cornilescu, J. L. Marquardt, M. Ottiger, A. Bax, Validation of protein structure from anisotropic carbonyl chemical shifts in a dilute liquid crystalline phase, Journal of the American Chemical Society 120 (27) (1998) 6836-6837.

[208] G. Cornilescu, A. Bax, Measurement of proton, nitrogen, and carbonyl chemical shielding anisotropies in a protein dissolved in a dilute liquid crystalline phase, Journal of the American Chemical Society 122 (41) (2000) 10143-10154.

[209] G. H. Park, R. W. Martin, D. Sakellariou, A. Pines, A. G. Shahkhatuni, A. A. Shahkhatuni, H. A. Panosyan, Variable angle spinning (VAS) NMR study of solvent effects in liquid crystalline solutions of ${ }^{13} \mathrm{C}-$ iodomethane, Chemical Physics Letters 399 (2004) 196-199.

[210] P. Diehl, Molecular structure from dipolar coupling, in: J. W. Emsley (Ed.), Nuclear Magnetic Resonance of Liquid Crystals, D. Reidel:, Dordrecht, The Netherlands, 1985, pp. 147-180.

[211] P. Diehl, Structure of rigid molecules dissolved in liquid crystalline solvents, in: D. M. Grant, R. K. Harris (Eds.), In Encyclopedia of Nuclear Magnetic Resonance, Vol. 7, John Wiley \& Sons, New York, 1996, pp. 4591-4602.

[212] E. E. Burnell, C. A. de Lange, Solute as probes of simplified models of orientational order, in: E. E. Burnell, C. A. de Lange (Eds.), NMR of Ordered Liquids, Kluwer Academic Publishers, Norwell, MA, 2003, pp. 221-240.

[213] P. Diehl, M. Reinhold, A. S. Tracey, E. Wullschleger, An interpretation of the anomalous results from a nuclear magnetic resonance study of ${ }^{13} \mathrm{C}$-methanol partially oriented in nematic liquid crystalline phases, Molecular Physics 30 (1975) 1781-1793.

[214] A. G. Shahkhatuni, A. A. Shahkhatuni, H. A. Panosyan, G. H. J. Park, R. W. Martin, A. Pines, NMR studies of ${ }^{13}$ C-iodomethane: Different behavior in thermotropic and lyotropic liquid crystals, Journal of Physical Chemistry A 108 (33) (2004) 6809-. 
[215] A. Saupe, Kernresonanzen in kristallinen Flüssigkeiten und in kristallinflüssigen Lösungen i,, Zeitschrift Fur Naturforschung Section A 19 (1964) 161-171.

[216] P. Fabre, C. Casagrande, M. Veyssié, H. Finkelmann, Elastic coefficients and twist viscosity in side-chain mesomorphic polymers, Physical Review Letters 53 (10) (1984) 993-996.

[217] T. Väänänen, J. Jokisaari, M. Seläntaus, Variable-angle spinning H1-NMR spectra of molecules in liquid-crystalline phases with positive and negative diamagnetic anisotropy, Chemical Physics Letters 129 (4) (1986) 399-402.

[218] R. A. Shapiro, Solid- and Solution-State NMR Techniques for the Investigation of Local Ordering in Membrane-Derived Solids via Measurement of Scaled Dipolar Couplings, Doctor of Philosophy, University of California, Irvine (2013).

[219] G. Kummerlöwe, S. L. Grage, I. Thiele, Christina M. Kuprov, A. S. Ulrich, B. Luy, Variable angle NMR spectroscopy and its application to the measurement of residual chemical shift anisotropy, Journal of Magnetic Resonance 209 (2011) 19-30.

[220] P. W. Kuchel, B. E. Chapman, N. Müller, W. A. Bubb, D. J. Philp, A. Torres, Apparatus for rapid adjustment of the degree of alignment of NMR samples in aqueous media: verification with residual quadrupolar splittings in ${ }^{23} \mathrm{Na}$ and ${ }^{133} \mathrm{Cs}$ spectra, Journal of Magnetic Resonance 180 (2006) $256-265$.

[221] C. Gayathri, N. V. Tsarevsky, R. R. Gil, Residual dipolar couplings (RDCs) analysis of small molecules made easy: fast and tuneable alignment by reversible compression/relaxation of reusable PMMA gels, Chemistry - A European Journal 16 (2010) 3622-3626.

[222] M. Teeäär, R. Alla, E. Lipmaa, Ordering in the nematic mesophase of a rapidly spinning sample, Organic Magnetic Resonance 19 (3) (1982) $134-137$.

[223] P. Lesot, Y. Gounelle, D. Merlet, A. Loewenstein, J. Courtieu, Measurement and analysis of the molecular ordering tensors of two enan- 
tiomers oriented in a polypeptide liquid crystalline system, Journal of Physical Chemistry 99.

[224] C. Aroulanda, P. Lesot, D. Merlet, J. Courtieu, Structural ambiguities revisited in two bridged ring systems exhibiting enantiotopic elements, using natural abundance deuterium NMR in chiral liquid crystals, Journal of Physical Chemistry A 107 (2003) 10911-10918.

[225] D. Merlet, J. W. Emsley, P. Lesot, J. Courtieu, The relationship between molecular symmetry and second-rank orientational order parameters for molecules in chiral liquid crystalline solvents, Journal of Chemical Physics 111 (15) (1999) 6890-6896.

[226] N. Tjandra, A. Bax, Direct measurement of distances and angles in biomolecules by NMR in a dilute liquid crystalline medium, Science 278 (5340) (1997) 1111-1114.

[227] P. Diehl, J. Jokisaari, J. Amrein, T. Väänänen, P. Pyykkö, Determination of the ${ }^{13} \mathrm{C}-{ }^{13} \mathrm{C}$ and ${ }^{13} \mathrm{C}-{ }^{15} \mathrm{~N}$ coupling constant anisotropies of acetonitrile partially oriented by nematic liquid crystals, Journal of Magnetic Resonance 48 (1982) 495-502.

[228] P. Diehl, J. Jokisaari, F. Moia, Determination of ${ }^{1} \mathrm{H}$ and ${ }^{13} \mathrm{C}$ chemical shift anisotropies. Comparison of methods using acetonitrile as a probe, Journal of Magnetic Resonance 49 (1982) 498-506.

[229] C. M. Thiele, Scaling the alignment of small organic molecules in substituted polyglutamates by variable-angle sample spinning, Angewandte Chemie International Edition 44 (2005) 2787 -2790.

[230] C. M. Thiele, Use of RDCs in rigid organic compounds and some practical considerations concerning alignment media, Concepts in Magnetic Resonance Part A 30A (2) (2007) 65-80.

[231] S. V. Dvinskikh, H. Zimmermann, A. Maliniak, D. Sandstrom, Separated local field spectroscopy of columnar and nematic liquid crystals, Journal of Magnetic Resonance 163 (1) (2003) 46-55.

[232] S. Dvinskikh, V. Castro, D. Sandstrom, Efficient solid-state NMR methods for measuring heteronuclear dipolar couplings in unoriented 
lipid membrane systems, Physical Chemistry, Chemical Physics 7 (2005) 607-613.

[233] B. M. Fung, J. Afzal, Carbon-13 NMR of liquid crystals. spinning near the magic angle with proton-proton dipolar decoupling, Journal of Physical Chemistry 108 (5) (1986) 1107-1108.

[234] P. Forster, B. M. Fung, Chain ordering in cyanobiphenyls and cyanophenylcyclohexanes, Journal of the Chemical Society-Faraday Transactions 284 (1988) 1083-1094.

[235] W. Guo, B. M. Fung, Determination of the order parameters of liquid crystals from C-13 chemical shifts, Journal of Chemical Physics 95 (6) (1991) 3917-3923.

[236] C. D. Poon, C. M. Wooldridge, B. M. Fung, Orientational ordering of 4-n-alkyl-oxy-4-cyanobiphenyls studied by 2D Carbon-13 NMR, Molecular Crystals and Liquid Crystals 157 (1) (1988) 303-318.

[237] W. Richter, B. M. Fung, M. Schadt, Orientational ordering in the nematic phase of 4-alkenyl-substituted bicyclohexylnitriles, Liquid Crystals 8 (1) (1990) 63-73.

[238] W. Guo, B. M. Fung, The effect of solutes on the orientational ordering of liquid-crystalline solvents, Liquid Crystals 9 (1) (1991) 117-126.

[239] T. H. Tong, B. M. Fung, J. P. Bayle, A database for the determination of orientational ordering of nine classes of liquid crystals using carbon13 chemical shifts, Liquid Crystals 22 (2) (1997) 165-169.

[240] J. W. Emsley, J. C. Lindon, NMR Spectroscopy Using Liquid Crystal Solvents, Pergamon Press, 1975.

[241] A. Pines, D. J. Ruben, S. Allison, Molecular ordering and even-odd effect in a homologous series of nematic liquid crystals, Physical Review Letters 33 (17) (1974) 1002-1005.

[242] F. Roussel, J. P. Bayle, M. A. Khan, B. M. Fung, O. Yokokohji, T. Shimizu, H. Koh, S. Kumai, Nematic ordering and crystal structure of liquid crystals containing a 4-chlorophenyldifluoroethylene unit, Liquid Crystals 26 (2) (1999) 251-260. 
[243] F. Perez, P. Judeinstein, J. P. Bayle, H. Allouchi, M. Cotrait, F. Roussel, B. M. Fung, Orientational ordering in some nematogens deviating from the classical rod-shape, Liquid Crystals 24 (4) (1998) 627-637.

[244] J. R. Garbow, D. P. Weitekamp, A. Pines, Bilinear rotation decoupling of homonuclear scalar interactions, Chemical Physics Letters 93 (1982) $504-515$.

[245] L. Ziani, J. Courtieu, D. Merlet, Visualisation of enantiomers via insertion of a BIRD module in $\mathrm{X}-\mathrm{H}$ correlation experiments in chiral liquid crystal solvent, Journal of Magnetic Resonance 183 (2006) 60-67.

[246] J. Farjon, D. Merlet, P. Lesot, J. Courtieu, Enantiomeric excess measurements in weakly oriented chiral liquid crystal solvents through 2D ${ }^{1} \mathrm{H}$ - selective refocusing experiments, Journal of Magnetic Resonance 158 (2002) 169.

[247] J. Farjon, J. P. Baltaze, P. Lesot, D. Merlet, J. Courtieu, Heteronuclear selective refocusing 2D NMR experiments for the spectral analysis of enantiomers in chiral oriented solvents, Magnetic Resonance in Chemistry 42 (2004) 594.

[248] C. L. Khetrapal, A. C. Kunwar, Determination of chemical shift anisotropy without a reference and of direct and indirect spin-spin couplings between heteronuclei, Chemical Physics Letters 82 (1981) 170-171.

[249] Y. Hiltunin, J. Jokisaari, determination of ${ }^{1} \mathrm{H}$ and ${ }^{13} \mathrm{C}$ shielding anisotropies for 1,3,5-trichlorobenzene in nematic liquid crystal mixtures, Journal of Magnetic Resonance 75 (2) (1987) 213-221.

[250] S. Vivekanandan, H. S. V. Deepak, G. A. N. Gowda, K. V. Ramanathan, C. L. Khetrapal, NMR spectra of mixed liquid crystals of opposite diamagnetic susceptibility anisotropies near critical point under variable angle sample spinning, Journal of Molecular Structure 602 (2002) 485-489.

[251] P. Lesot, F. Nielsen, J. M. Ouvrard, J. Courtieu, Multiple-pulse COSY NMR spectroscopy of oriented molecules in thermotropic cholesterics, Journal of Physical Chemistry 98 (1994) 12849-12855. 
[252] P. Lesot, J. W. Emsley, J. Courtieu, The magnetohydrodynamics of a chiral nematic liquid crystal: the existence of two critical speeds revealed by deuterium NMR, Liquid Crystals 25 (1) (1998) 123-127.

[253] D. McElheny, M. Zhou, L. Frydman, Two-dimensional dynamicdirector C-13 NMR of liquid crystals, Journal of Magnetic Resonance 148 (2001) 436.

[254] R. H. Havlin, G. H. J. Park, A. Pines, Two-dimensional NMR correlations of liquid crystals using switched angle spinning, Journal of Magnetic Resonance 157 (1) (2002) 163-169.

[255] R. Havlin, G. Park, T. Mazur, A. Pines, Using switched angle spinning to simplify NMR spectra of strongly oriented samples, Journal of the American Chemical Society 125 (26) (2003) 7998-8006.

[256] C. R. Sanders, J. P. Schwonek, Characterization of magnetically orientable bilayers in mixtures of dihexanoylphosphatidylcholine and dimyristoylphosphatidylcholine by solid-state NMR, Biochemistry 31 (37) (1992) 8898-8905.

[257] I. Marcotte, M. Auger, Bicelles as model membranes for solid- and solution-state NMR studies of membrane peptides and proteins, Concepts in Magnetic Resonance Part A 24A (1) (2005) 17-37.

[258] P. Ram, J. H. Prestegard, Magnetic-field induced ordering of bile salt phospholipid micelles - new media for NMR structural investigations, Biochimica Et Biophysica Acta 940 (2) (1988) 289-294.

[259] C. R. Sanders, B. J. Hare, K. P. Howard, J. H. Prestegard, Magnetically-oriented phospholipid micelles as a tool for the study of membrane-associated molecules, Progress in Nuclear Magnetic Resonance Spectroscopy 26 (1994) 421-444.

[260] M. Ottiger, A. Bax, Characterization of magnetically oriented phospholipid micelles for measurement of dipolar couplings in macromolecules, Journal of Biomolecular NMR 12 (3) (1998) 361-372.

[261] E. K. Tiburu, D. M. Moton, G. A. Lorigan, Development of magnetically aligned phospholipid bilayers in mixtures of palmitoylstearoylphosphatidylcholine and dihexanoylphosphatidylcholine by 
solid-state NMR spectroscopy, Biochimica Et Biophysica ActaBiomembranes 1512 (2) (2001) 206-214.

[262] K. J. Crowell, P. M. Macdonald, Surface charge response of the phosphatidylcholine head group in bilayered micelles from phosphorus and deuterium nuclear magnetic resonance, Biochimica Et Biophysica ActaBiomembranes 1416 (1-2) (1999) 21-30.

[263] J. Struppe, J. A. Whiles, R. R. Vold, Acidic phospholipid bicelles: A versatile model membrane system, Biophysical Journal 78 (1) (2000) $281-289$.

[264] M. Ottiger, A. Bax, Bicelle-based liquid crystals for NMR-measurement of dipolar couplings at acidic and basic $\mathrm{pH}$ values, Journal of Biomolecular NMR 13 (2) (1999) 187-191.

[265] S.-H. Park, S. J. Opella, Triton x-100 as the "short-chain lipid" improves the magnetic alignment and stability of membrane proteins in phosphatidylcholine bilayers for oriented sample solid-state nmr, Journal of the American Chemical Society 132 (2010) 12552-12553.

[266] M.-P. Nieh, V. A. Raghunathan, C. J. Glinka, T. Harroun, G. Pabst, J. Katsaras, Magnetically alignable phase of phospholipid "bicelle" mixtures is a chiral nematic made up of wormlike micelles, Langmuir 20 (2004) 7893-7897.

[267] J. D. Gross, D. E. Warschawski, R. G. Griffin, Dipolar recoupling in MAS NMR: A probe for segmental order in lipid bilayers, Journal of the American Chemical Society 119 (4) (1997) 796-802.

[268] S. Dvinskikh, U. Durr, K. Yamamoto, A. Ramamoorthy, A highresolution solid-state NMR approach for the structural studies of bicelles, Journal of the American Chemical Society 128 (2006) 6326-6327.

[269] R. S. Prosser, S. A. Hunt, J. A. DiNatale, R. R. Vold, Magnetically aligned membrane model systems with positive order parameter: Switching the sign of S-zz with paramagnetic ions, Journal Of The American Chemical Society 118 (1) (1996) 269-270.

[270] K. P. Howard, S. J. Opella, High-resolution solid-state NMR spectra of integral membrane proteins reconstituted into magnetically oriented 
phospholipid bilayers, Journal of Magnetic Resonance Series B 112 (1) (1996) 91-94.

[271] R. S. Prosser, J. S. Hwang, R. R. Vold, Magnetically aligned phospholipid bilayers with positive ordering: A new model membrane system, Biophysical Journal 74 (5) (1998) 2405-2418.

[272] A. I. Kishore, J. H. Prestegard, Molecular orientation and conformation of phosphatidylinositides in membrane mimetics using variable angle sample spinning (VASS) NMR, Biophysical Journal 85 (6) (2003) 38483857 .

[273] J. Struppe, R. R. Vold, Dilute bicellar solutions for structural NMR work, Journal of Magnetic Resonance 135 (2) (1998) 541-546.

[274] R. S. Prosser, V. B. Volkov, I. V. Shiyanovskaya, Solid-state NMR studies of magnetically aligned phospholipid membranes: taming lanthanides for membrane protein studies, Biochemistry And Cell BiologyBiochimie Et Biologie Cellulaire 76 (2-3) (1998) 443-451.

[275] N. Lancelot, K. Elbayed, M. Piotto, Applications of variable-angle sample spinning experiments to the measurement of scaled residual dipolar couplings and N-15 CSA in soluble proteins, Journal of biomolecular NMR 33 (3) (2005) 153-161.

[276] J. E. Ollerenshaw, V. Tugarinov, L. E. Kay, Methyl TROSY: explanation and experimental verification, Magnetic Resonance in Chemistry 41 (2003) 843-852.

[277] G. Zandomeneghi, M. Tomaselli, P. T. F. Williamson, B. H. Meier, NMR of bicelles: orientation and mosaic spread of the liquid-crystal director under sample rotation, Journal of Biomolecular NMR 25 (2) (2003) 113-123.

[278] N. Boden, S. A. Corne, K. W. Jolley, Lyotropic mesomorphism of the cesium pentadecafluorooctanoate/water system: high-resolution phase diagram, Journal of Physical Chemistry 91 (1987) 4092-4105.

[279] J. P. Bayle, F. Perez, J. Courtieu, Inertial effects in rotating lyotropic liquid crystals, Liquid Crystals 3 (1988) 753-758. 
[280] G. Zandomeneghi, P. T. F. Williamson, A. Hunkeler, B. H. Meier, Switched-angle spinning applied to bicelles containing phospholipidassociated peptides, Journal of Biomolecular NMR 25 (2) (2003) 125132.

[281] C. Glaubitz, A. Watts, Magic Angle-Oriented Sample Spinning (MAOSS): A new approach toward biomembrane studies, Journal of Magnetic Resonance 130 (305-316).

[282] F. Moll, T. A. Cross, Optimizing and characterizing alignment of oriented lipid bilayers containing gramicidin D, Biophysical Journal 57 (1990) 351-362.

[283] D. Sakellariou, C. A. Meriles, R. W. Martin, A. Pines, High-resolution NMR of anisotropic samples with spinning away from the magic angle, Chemical Physics Letters 377 (2003) 333-339.

[284] N. Lancelot, K. Elbayed, A. Bianco, M. Piotto, Measurement of scaled residual dipolar couplings in proteins using variable-angle sample spinning, Journal of Biomolecular NMR 29 (3) (2004) 259-269.

[285] G. Morris, R. Freeman, Enhancement of nuclear magnetic resonance signals by polarization transfer, Journal of the Americal Chemical Society 101 (1979) 760-762.

[286] W. Tang, A. A. Nevzorov, Repetitive cross-polarization contacts via equilibration-re-equilibration of the proton bath: Sensitivity enhancement for NMR of membrane proteins reconstituted in magnetically aligned bicelles, Journal of Magnetic Resonance 212 (1) (2011) 245248.

[287] V. A. Mandelshtam, H. S. Taylor, A. J. Shaka, Application of the Filter Diagonalization Method to One- and Two-Dimensional NMR Spectra, Journal of Magnetic Resonance 133 (2) (1998) 304-312.

[288] L. Frydman, T. Scherf, A. Lupulescu, The acquisition of multidimensional NMR spectra within a single scan, Proceedings of the National Academy of Sciences of the United States of America 99 (25) (2002) $15858-15862$. 
[289] A. Tal, L. Frydman, Single-scan multidimensional magnetic resonance, Progress in Nuclear Magnetic Resonance Spectroscopy 57 (3) (2010) 241-292.

[290] P. Giraudeau, T. Montag, B. Charriera, C. M. Thiele, Fast access to residual dipolar couplings by single-scan 2D NMR in oriented media, Magnetic Resonance in Chemistry 50 (2012) S53-S57. 